\title{
Plutonic rocks of the Median Batholith in southwest Fiordland, New Zealand: field relations, geochemistry, and correlation
}

\author{
A. H. ALLIBONE* \\ School of Earth Science \\ James Cook University \\ Townsville Q4811, Australia \\ rodinian@msn.com \\ I. M. TURNBULL \\ A. J. TULLOCH \\ GNS Science \\ Private Bag 1930 \\ Dunedin 9054, New Zealand \\ A. F. COOPER \\ Department of Geology \\ University of Otago \\ PO Box 56 \\ Dunedin 9054, New Zealand
}

*Present address: Rodinian Pty Ltd, PO Box 1804, Fyshwick, ACT 2609, Australia.

\begin{abstract}
This paper provides a first description of all major plutonic rock units between Resolution Island and Lake Poteriteri in southwest Fiordland. Plutonic rocks, of which c. $95 \%$ are granitoids, comprise c. $60 \%$ of the basement in southwest Fiordland. Approximately $50 \%$ of the plutonic rocks were emplaced between c. 355 and $348 \mathrm{Ma}, 5 \%$ at c. $164 \mathrm{Ma}, 25 \%$ between c. 140 and $125 \mathrm{Ma}$, and $20 \%$ between c. 125 and $110 \mathrm{Ma}$. These episodes of plutonism occurred in response to terrane amalgamation, continental thickening, and subduction along the convergent margin of Gondwana. Correlatives of Devonian plutonic rocks which occur in Nelson are absent from the area described here.

A wide variety of plutonic rocks were emplaced at c. 355$348 \mathrm{Ma}$. These include relatively small plutons of K- and $\mathrm{Rb}$-rich gabbro-diorite and members of at least three distinct suites of granitoids. Plutons of two-mica \pm garnet granodiorite, granite, and minor tonalite share affinities with the S-type Ridge Suite and are the most widespread c. 355-348 Ma old granitoids in southern Fiordland. Plutons rich in $\mathrm{Ca}, \mathrm{Fe}$ and $\mathrm{Zr}$, depleted in $\mathrm{K}$ and $\mathrm{Na}$, and containing quartz diorite, tonalite, and minor granodiorite with the unusual assemblage red-brown biotite, garnet \pm hornblende \pm clinopyroxene also occur widely in southern Fiordland. These plutons are similar to peraluminous A-type granitoids, indicating A as well as I and S-type plutonism occurred in the Western Province at this time. The Newton River and Mt Evans Plutons have no correlatives amongst c. 355-348 Ma granitoids in southern Fiordland, but their chemistry is similar to that of the older Karamea Suite.
\end{abstract}

G07002; Online publication date 21 September 2007 Received 19 February 2007; accepted 30 July 2007
Three regional-scale metasedimentary units-locally fossiliferous Fanny Bay Group Buller Terrane rocks in southern Fiordland, Edgecumbe and Cameron Group Takaka Terrane rocks in south-central Fiordland, and undifferentiated Deep Cove Gneiss high-grade metasedimentary rocks of western Fiordland-are all stitched by c. $355-348$ Ma old plutons, indicating they have been in close proximity since at least c. 355-348 Ma. In south-central Fiordland, c. 355-348 Ma old plutons cut across fabrics defined by upper amphibolite facies mineral assemblages, indicating low pressure/high temperature metamorphism in this area before this time.

The c. 164 Ma old leucocratic Lake Mike Granite is a unique pluton in southwest Fiordland with no obvious correlatives. Plutons emplaced between c. 140 and $125 \mathrm{Ma}$ are similar to the Rahu Suite, although isotopic data are required to confirm this correlation. Rahu Suite plutonism may therefore have begun by c. $140 \mathrm{Ma}$, rather than c. $120 \mathrm{Ma}$ as previously suggested. Plutons emplaced between c. 125 and $110 \mathrm{Ma}$ have high $\mathrm{Sr} / \mathrm{Y}$ ratios comparable with the Separation Point Suite. They occur in both an outboard location around Lake Poteriteri and an inboard location around the western end of Dusky Sound. The c. 115 Ma two-mica garnet granites of the Anchor Island Intrusives \#2 probably formed by partial melting of adjacent ortho- and paragneisses, indicating that upper amphibolite facies metamorphism in western Dusky Sound occurred during the Early Cretaceous.

The Dusky Fault does not pass directly out to the coast through outer Dusky Sound as previously mapped. Instead it merges with the major northeast-striking Lake Fraser Fault at Cascade Cove, which crosses the outer coast near West Cape. The Last Cove Fault is a minor structure which cannot be traced beyond Last Cove rather than a major fault of regional extent as has been previously suggested.

Keywords Fiordland; Anchor Island, Tower Intrusives; Bald Peaks, Big, Brothers, Cascade, Fannin, Five Fingers, Grace Burn, Houseroof, Mouat, Indian Island, Jeanie, Lake 773, Mid Poteriteri, Mt Evans, Newton River, Red Head, Spot 59, Treble Mountain, Prices, Widgeon, Trevaccoon Plutons; Lake Mike, Revolver, North Port, Lake Monk Granites; Staircase Tonalite; Thundercleft Quartz Diorite; Only Islands Diorite; dikes; migmatites; gabbro; diorite; tonalite; granodiorite; granite; Ridge, Tobin, Separation Point, Rahu Suites; Median Batholith; Dusky, Lake Fraser Fault; new lithologic names

\section{INTRODUCTION}

This paper provides a first description of all major gabbroic, dioritic, and granitoid plutons in southwest Fiordland between Resolution Island, Preservation Inlet, Lake Poteriteri, and the head of Dusky Sound (Fig. 1). This remote part of Fiordland has recently been mapped as part of the New Zealand Public Good Science Fund (PGSF)-funded QMAP 1:250 000 Geological Map of New Zealand programme. 


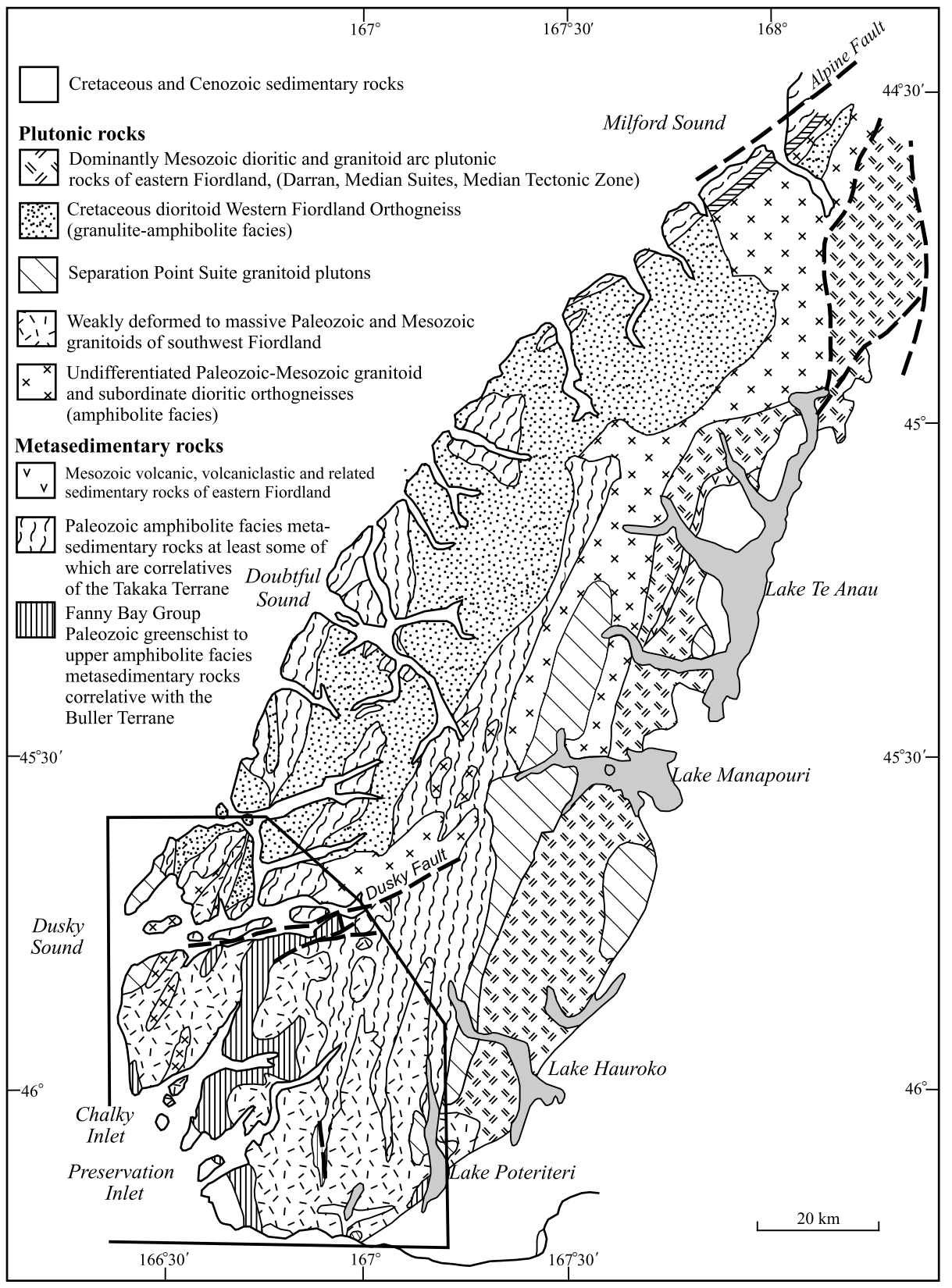

Fig. 1 Regional geology of Fiordland (after Oliver \& Coggon (1979), Bradshaw (1990), Marcotte etal.(2005), and Allibone, Turnbull and Jongens (unpubl. data)). The area mapped (outlined) is confined to the Western Province (Landis \& Coombs 1967), and includes parts of the Western and Southwest Fiordland regions of Oliver \& Coggon (1979), the inferred western extension of the Dusky Fault, and the likely position of the boundary between the Buller and Takaka Terranes in south-central Fiordland.

Previous mapping has been restricted to parts of the coastal section, a reconnaissance of some inland areas (Bishop 1986), and local detailed mapping in the northeast part of the area described here (Ward 1984; Powell 2006). In this paper we introduce and describe the plutonic units included in this part of the QMAP Fiordland sheet, discuss their suite affinities, and establish correlations with plutonic rocks in other parts of New Zealand.

\section{Regional geologic setting}

The Fiordland region contains many of New Zealand's highgrade metamorphic and plutonic rocks. These rocks and their correlatives in Westland, Nelson, and Stewart Island comprise a small segment of the Paleozoic margin of Gondwana, and the remains of a Mesozoic volcanic arc developed along and adjacent to the margin of Gondwana. Rocks described in this paper are located wholly within the area that once comprised part of Paleozoic Gondwana. This is indicated by the presence of Paleozoic metasedimentary rocks throughout the area described (Fig. 2). Since 1967, these rocks have been included in the Western Province of the New Zealand basement (Landis \& Coombs 1967).

Plutonic rocks in southwest Fiordland provide a record of the nature, extent, and timing of plutonism in this section of Gondwana between the Carboniferous and Cretaceous. Previous work in Nelson and Stewart Island has divided plutonic rocks correlative with those in southwest Fiordland into several Devonian, Carboniferous, and Cretaceous petrogenetic suites (Table 1). The Rahu Suite (Tulloch 1983) as used here also includes the Deutgam Suite (Waight et al. 1998) of the Hohonu Ranges, included in the Rahu Suite by Tulloch \& Braithwaite (1986). Emplacement of the various suites reflects repeated episodes of continental thickening, terrane amalgamation, subduction, and post-subduction extension along the margin of Gondwana between the Devonian and Cretaceous. 


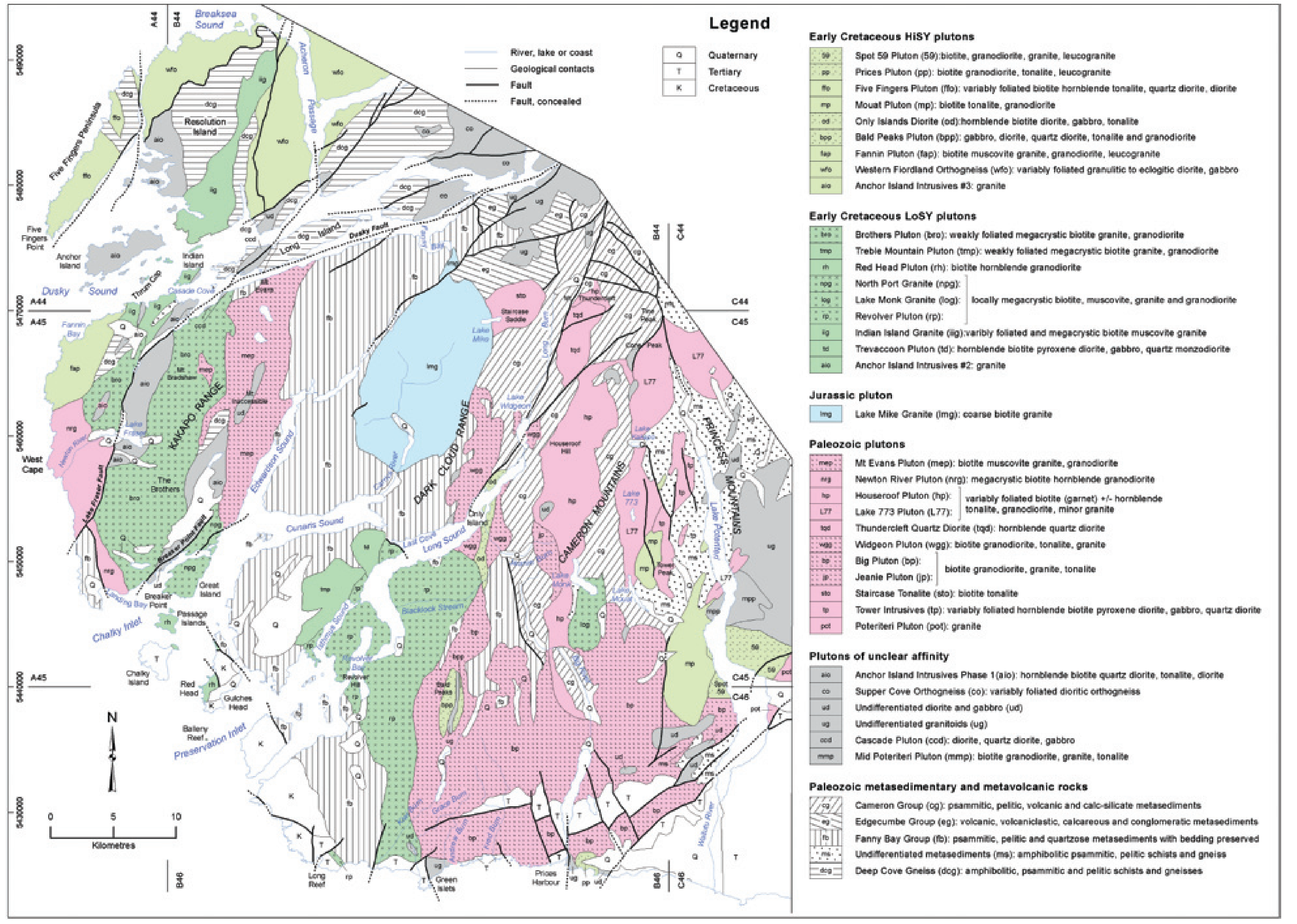

Fig. 2 Geology of the area between Resolution Island and Lake Poteriteri, with topographic features mentioned in the text. All major plutons in southwest Fiordland are shown. Anchor Island Intrusives phases \#1-\#3 cannot be differentiated at this scale. Most plutons
form elongate N to NNE-trending intrusions separated by belts of metasedimentary rock. Cameron and Edgecumbe Groups are probable correlatives of the Takaka Terrane. The Fanny Bay Group is a correlative of the Buller Terrane. Supper Cove Orthogneiss (co) and Western Fiordland Orthogneiss (wfo) in the northern part of the map are not discussed in this paper. 
Mesozoic plutonic rocks described here are correlatives of the Separation Point and Rahu Suites (Tulloch 1983), which form the inboard part of the Median Batholith (Mortimer et al. 1999). Plutons here included in the Separation Point Suite would also be included in the Tutoko Complex under the definition proposed by Wandres \& Bradshaw (2005). Mesozoic plutons in southwest Fiordland are not correlatives of those which comprise the outboard part of the Median Batholith. These rocks have previously been included in the Median Tectonic Zone (Kimbrough et al. 1993, 1994; Muir et al. 1998), Median Suite (Mortimer \& Tulloch 1996; Tulloch \& Kimbrough 2003), Darran Suite (Muir et al. 1998) and Tutoko Complex (Wandres \& Bradshaw 2005) (Fig. 1). As a result, the complex history and inter-relationships of these various overlapping units are not discussed here.

The area described here includes all of the Southwest Fiordland region, and parts of the Western and Central Fiordland regions as defined by Oliver \& Coggon (1979). It also includes the western part of the Dusky Fault, and the Last Cove Fault, both previously inferred to be important regional boundaries in southwest Fiordland (Wood 1960; Oliver \& Coggon 1979; House et al. 2005). In southwest Fiordland, plutonic rocks comprise c. $60 \%$ of the basement. The remainder consists of Paleozoic Western Province metasedimentary rocks which (Fig. 1) include likely correlatives of both the Buller and Takaka Terranes in Westland and Nelson (e.g., Mortimer 2004). In southwest Fiordland, the Buller Terrane is represented by the Fanny Bay Group, whereas the Takaka Terrane is represented by the Cameron and Edgecumbe Groups (Fig. 2) (Ward 1986; Powell 2001, 2006), informal units which have been adopted by Cooper (1989) and which will be used by the QMAP programme. North of the Dusky Fault, higher grade metasedimentary and metavolcanic country rocks are mapped as Deep Cove Gneiss, a diverse unit of Paleozoic age (Oliver 1980; Gibson 1982; Ward 1984). The presence of calc-silicate and marble, amphibolite, psammite, pelite, minor quartzite, and rare conglomerate (Ward 1984) suggests an affinity with the Takaka rather than Buller Terrane.

\section{Previous work}

Preliminary observations on the rocks along the southwest Fiordland coast were recorded by Hector (1863) and Park (1921). Benson (1934) and Benson \& Bartrum (1935) provided the first detailed petrographic descriptions of granitoid and dioritic rocks around the shorelines of Chalky and Preservation Inlets and Edwardson, Cunaris, and Long Sounds. Benson (1934) also described the low-grade Paleozoic metasediments of southwest Fiordland and recognised Ordovician graptolitic faunas.

Fiordland rocks were originally included in a "Fiordland Complex" (Turner 1935; Wood 1960). Wood (1960) included many of the granitoid rocks in southwest Fiordland in the extensive "Kakapo Granite", named from the Kakapo Range between Edwardson and Dusky Sounds. Oliver \& Coggon (1979) suggested that variations in the magnetic susceptibility of different parts of the Kakapo Granite sensu Wood (1960) mean that it comprises several individual plutons. Bishop (1986) applied the name Kakapo Granite to all granitoid rocks in the southwest part of Fiordland, and defined a type section along the Long Sound shoreline below Revolver Hill, $20 \mathrm{~km}$ southeast of the Kakapo Range. Reconnaissance Rb-Sr and U-Pb dating (Aronson 1965, 1968; Powell \& Kimbrough 1987; Muir et al. 1998) confirmed that the Kakapo Granite as mapped by Wood (1960) and Bishop (1986) includes both Carboniferous and Early Cretaceous plutons. Bishop's (1986) Kakapo Granite type section is within the Revolver Granite, a name coined by Powell \& Kimbrough (1987) to distinguish their dated rock from other plutons of different ages included within the Kakapo Granite sensu Wood (1960) and Bishop (1986).

Ward (1984) and Powell (2006) described in detail low to high grade metasedimentary rocks in the north and east of the area covered by this paper. They also mapped the intrusive sequences, some of which were dated by Davids (1999) in a study of the Dusky Fault.

Turnbull \& Uruski (1995) mapped a variety of granitoid and dioritic rocks along the southwest shore of Lake Poteriteri and in the mountains immediately to the west. The full extent and character of these units is described here. Muir et al. (1998), Ewing (2003), and Gollan (2006) obtained Devonian-Carboniferous ages for several samples of the "Kakapo Granite" sensu Bishop (1986), here included in the Big Pluton, on the ridges between Lake Poteriteri and Big River. No other descriptions of plutonic rocks have been published from the area mapped, although unpublished student theses cover small areas in the southwest (Badger 1973; Lindqvist 1975).

\section{This study}

Intrusive rocks described here have generally been mapped as plutons. Individual plutons may comprise a single homogeneous rock type or a variety of rocks with variable compositions and textures. However, all rocks within a single pluton have field relationships and geochemistry that suggest

Table 1 Names, ages, and selected characteristics of granitoid suites previously defined in those parts of Nelson, Westland, Fiordland, and Stewart Island that correlate with the area described in this paper. Data and nomenclature are from Tulloch (1983), Tulloch \& Braithwaite (1986), Tulloch (1988), Tulloch \& Rabone (1993), Tulloch \& Kimbrough (2003), Tulloch et al. (2003), Cooper \& Tulloch (1992); Muir et al. (1996a,b), Waight et al. (1998), and Tulloch \& Allibone (unpubl. analyses from Stewart Island). Other granitoid suites not represented in the area mapped are not listed here.

\begin{tabular}{|c|c|c|c|c|c|c|}
\hline Suite & Age & Type & Terrane & $\mathrm{Sr} / \mathrm{Y}$ & $\mathrm{Rb} / \mathrm{Sr}$ & $\mathrm{SiO}_{2}(\%)$ \\
\hline Karamea & $382-369$ & S-type & Buller & $1-7$ & $0.9-20$ & $66-76$ \\
\hline Paringa & $368-360$ & I-type & Buller and Takaka & $25-100$ & $0.01-0.3$ & $53-75$ \\
\hline Ridge & $356-342$ & S-type & Takaka & $6-31$ & $0.1-1.1$ & $63-74$ \\
\hline Tobin & $349-341$ & I-type & Buller and Takaka & $2-34$ & $0.06-1.9$ & $52-76$ \\
\hline Foulwind & $320-290$ & A-type & Buller and Takaka & $0-19$ & $0.3-19$ & $63-76$ \\
\hline Rahu & $120-100$ & I/S-type & Buller & $10-70$ & $0.15-1.0(22)$ & $63-75$ \\
\hline $\begin{array}{l}\text { Separation Point } \\
\text { (excluding WFO) }\end{array}$ & $125-105$ & I-type & Buller and Takaka & $\begin{array}{r}40-700 \\
(2250)\end{array}$ & $0.01-2.0(4.0)$ & $60-77$ \\
\hline
\end{tabular}


they are derived from a single magma or several closely related batches of magma (e.g., Cox \& Allibone 1995; Glazner et al. 2004). The nomination of a type locality rather than a single type outcrop for each plutonic unit reflects their variable internal character. Only the most homogeneous plutons have been given names that indicate a specific composition (e.g., Lake Mike Granite). Units that comprise numerous intrusive phases which cannot be separated at the 1:50 000 mapping scale are described as "Intrusives". A pluton-based mapping approach allows individual intrusions to be correlated with petrogenetic suites as geochemical and geochronological data become available, whereas this would not be the case if composite units such as "Kakapo Granite" were retained. Key characteristics of each pluton are summarised in Table 2. In many places there are no suitable geographic names for new units, and previously used but informal geographic names, or topographic features from NZMS 260 1:50 000 map sheets, have been applied. In this remote part of Fiordland, many nominated type localities can only be easily reached by helicopter or boat.

Geochronological data are from U-Pb dating of zircon and monazite. Analyses were undertaken by Jahandar Ramezani at Massachusetts Institute of Technology by thermal ionisation mass spectrometry (TIMS) on single grains. Average ${ }^{206} \mathrm{~Pb} /{ }^{238} \mathrm{U}$ and ${ }^{207} \mathrm{~Pb} /{ }^{235} \mathrm{U}$ errors for all individual analyses in the dataset are 0.10 and $0.16 \%, 2 \sigma$, respectively. Full data for the Paleozoic plutons are currently in review (Tulloch et al. "Paleozoic plutonism in western New Zealand and relationship to Gondwana-margin magmatism in eastern Australia and Antarctica" submitted to Geological Society of America Bulletin 2007) and is available from the authors on request. Mesozoic age data are reported in Tulloch \& Ramezani (2007) or are available from the authors. Some ages are based on one or two single crystals. Those ages comprising two concordant, near-equivalent single grains are reported as c. Xxx.x Ma. Those analyses where two single grains do not meet both these criteria are reported as c. Xxx Ma, reflecting their reconnaissance status.

Sample numbers prefixed by $P$ are housed in the PETLAB collection of GNS Science (http://data.gns.cri.nz/pet/). Those samples with an OU prefix are lodged with the Department of Geology, University of Otago. Plagioclase compositions were determined optically. Grid references are given in terms of the metric New Zealand topographic map series NZMS 260 1:50 000 .

\section{PALEOZOIC PLUTONS}

Nine Paleozoic granitoid plutons, extensive related dike swarms, and a complex Paleozoic gabbro-diorite body have been mapped in southwest Fiordland (Fig. 2). With two exceptions, these granitoid plutons are confined to a northtrending zone c. $20 \mathrm{~km}$ wide and at least $55 \mathrm{~km}$ long in southcentral Fiordland, between Long Sound and Lake Poteriteri (Fig. 2). Within this c. $20 \mathrm{~km}$ wide zone, north-striking belts of Paleozoic amphibolite facies metasedimentary rocks separate each pluton. These belts are often pervasively intruded by swarms of granitoid dikes derived from the adjacent Paleozoic plutons, while xenoliths of metasedimentary rock occur widely within each pluton. None of the seven granitoid plutons in south-central Fiordland were observed crosscutting each other. Mutually crosscutting relationships characterise contacts between the gabbro-diorite body and one of the granitoid plutons, consistent with all the Paleozoic intrusions in south-central Fiordland having broadly the same age. Two additional Paleozoic plutons occur farther to the west - one in the Kakapo Range and another along the west coast between Chalky Inlet and Dusky Sound (Fig. 2).

\section{Tower Intrusives}

Gabbroic, dioritic, and locally tonalitic rocks intrude Paleozoic metasedimentary rocks in the eastern Cameron Mountains, where they form a near-contiguous mass, named the Tower Intrusives (new name), which extends northeast from Lake Mouat to Lake Kakapo. Tower Intrusives from the type locality at Tower Peak (Fig. 2) are dominated by weakly foliated to gneissic, fine to medium grained hornblendebiotite diorite, quartz diorite, and minor tonalite. Elsewhere, the Tower Intrusives include areas up to $1 \mathrm{~km}$ across of distinctive red weathering, very coarse gabbro, and rare pyroxenite (P73523, 73508), cut by conspicuous granitoid dikes and coarse hornblende pegmatite. Deformed gabbro is locally recrystallised to a coarse-grained hornblende-garnetplagioclase gneiss (P73516, 73425) or partly altered and recrystallised at a lower metamorphic grade to actinolite and serpentinite (P73484). Altered gabbro and talc-chlorite schist also occur along narrow discrete shear zones. U-Pb zircon dating of a tonalitic phase from the pluton indicates an emplacement age of c. 351 Ma.

\section{Big, Widgeon and Jeanie Plutons, Staircase Tonalite, and related dike swarms}

Big Pluton (new name) is the largest Paleozoic granitoid pluton in south-central Fiordland. It underlies much of the western and southern parts of the Cameron Mountains, including the catchments of the Kiwi Burn, Cavendish River, and Big River from where it is named (Fig. 2). It extends to the south coast, and eastwards to Lake Poteriteri. The margins of the pluton are commonly marked by xenolith-rich zones up to $1 \mathrm{~km}$ wide in which metasedimentary material comprises c. $50 \%$ of the rock. That part of the Poteriteri Pluton mapped by Turnbull \& Uruski (1995) west of Lake Poteriteri is now included in Big Pluton. U-Pb SHRIMP dating of zircons from the Big Pluton implies a late Devonian or Carboniferous emplacement age between c. 380 and 340 Ma (Muir et al. 1998, KAK-1 "Kakapo Granite"; Ewing 2003, KAK-1, KAK2, KAK3, "Kakapo Granite”; Gollan 2006, Big Pluton). U-Pb TIMS zircon dating of P70787 yielded an age of $354.35 \pm 0.14 \mathrm{Ma}$.

Several smaller plutons in south-central Fiordland comprise similar granitoid rocks to the Big Pluton, and share the same field relationships with the intervening rocks. Widgeon Pluton (named from the informal "Widgeon Granite Gneiss" of Ward 1984) crops out north of Long Sound in the western Dark Cloud Range (Fig. 2). The type locality is around B45/350630 on the tops above Lake Widgeon (hence the name). U-Pb TIMS dating of zircons from the Widgeon Pluton indicates an emplacement age between 340 and 390 Ma. Jeanie Pluton (new name) forms a xenolithchoked intrusion in the headwaters of the west branch of the Big River and the Jeanie Burn (Fig. 2), with a type locality on the ridge between the two streams around B45/402506. Staircase Tonalite (new name, after Ward 1984) crops out over c. $14 \mathrm{~km}^{2}$ in the easternmost Dark Cloud Range around Staircase Saddle at B45/374690, the type locality. Dike swarms which surround these plutons (Fig. 2) pervasively intrude the adjacent metasedimentary rocks and locally link 
Fig.3 Compositions of Paleozoic plutonic rocks in southwest Fiordland derived from modal analyses and calculated mesonorms (LeMaitre 1989) plotted on a QAP diagram (Streckeisen 1976). Fractionation trends of the Paleozoic granitoid plutons extend from quartz-diorite through tonalite and granodiorite to granite (sensu stricto). Alkaline rocks rich in K-feldspar are absent apart from a single K-feldsparrich sample of the Big Pluton (not shown). Arrows link modal and mesonorm analyses of the same samples.

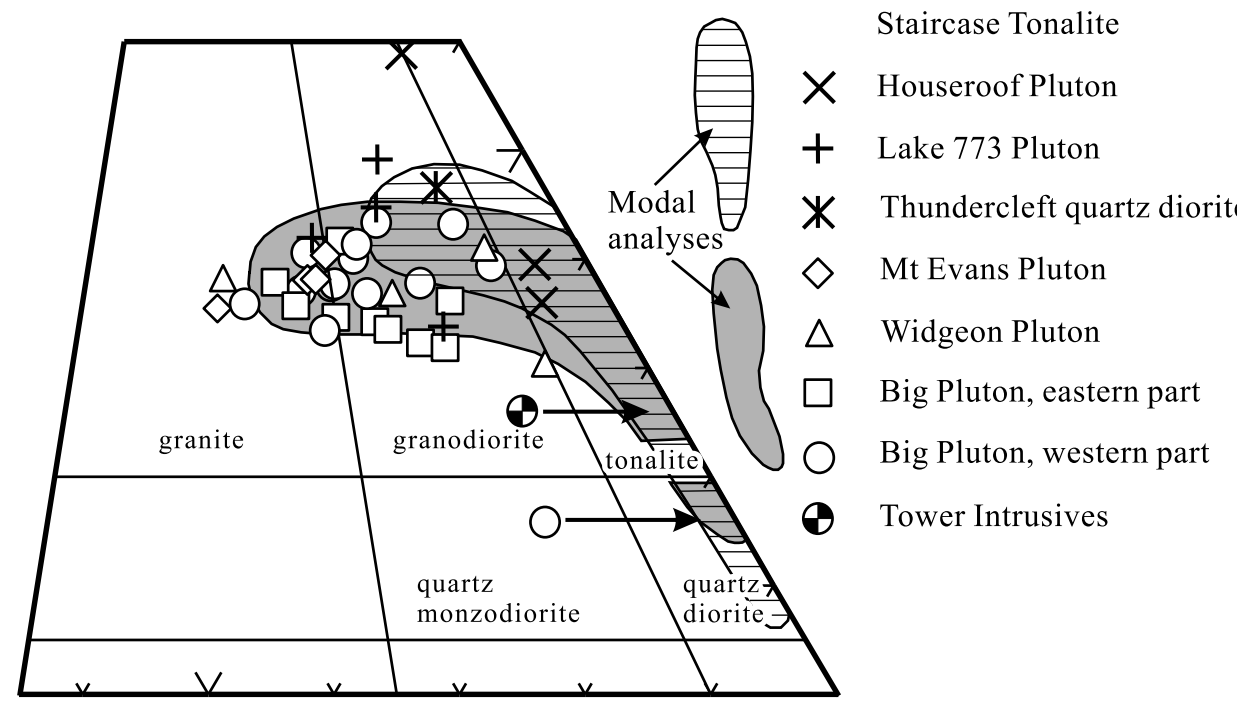

the Jeanie, Widgeon, and Big Plutons, implying that these three intrusions are apophyses of a larger body at depth. A further swarm of similar dikes cuts metasedimentary rocks around the northern part of Lake Poteriteri and may be derived from a large underlying correlative pluton (Fig. 2).

Massive to weakly foliated, medium to coarse grained white granodiorite, and granite with minor tonalite, forms c. $99 \%$ of the Big, Widgeon and Jeanie Plutons, Staircase Tonalite, and related dike swarms (Fig. 3). K-feldspar-rich alkali feldspar granite and syenite form a minor part of the Big Pluton (P73131). Equigranular textures dominate each unit, with K-feldspar megacrystic textures largely restricted to some of the easternmost parts of the Big Pluton. Deep red-brown biotite is the dominant mafic mineral in all four plutons and related dike swarms (P73489, 73486). In the easternmost $20 \%$ of the Big Pluton, biotite has a darker brown colour. Muscovite and garnet are common accessory minerals. Dark brown biotite in the easternmost part of the Big Pluton and some samples of the Widgeon Pluton can be accompanied by accessory titanite, allanite, and magmatic epidote rather than garnet and/or muscovite. Rare samples along this mineralogical boundary within the Big Pluton contain minerals from both sub-assemblages (e.g., P70778, garnet-muscovite-titanite; P70798, muscovite-epidote). However, no clear sharp intrusive contact or marked change in texture or geochemistry coincides with these mineralogical changes in the Big Pluton, implying that rocks with both assemblages form part of a single, mineralogically zoned body rather than two separate intrusions. Where titanite is present in trace amounts in the western part of the Big Pluton and the Widgeon Pluton, it is restricted to the most biotite-rich samples. Coarse apatite and zircon are present throughout all four intrusions. The low magnetic susceptibility of Big Pluton samples (Table 2) suggests that the opaque oxide is ilmenite.

Primary igneous interstitial and subhedral textures are widely preserved in all four plutons and related dikes. Deformation is generally restricted to minor sub-grain development, serration of feldspar grain boundaries, and recrystallisation of coarser igneous quartz grains to aggregates of finer grains. It is not clear whether the local weak alignment of biotite is a primary magmatic feature or reflects weak postcrystallisation deformation. Aligned biotite locally defines a weak but measurable foliation and/or lineation, which is often parallel to the margins of metasedimentary rafts. Within the Big Pluton, foliation generally strikes between NNE and NNW and dips moderately to steeply to the west. A steep west-plunging lineation is locally present in the most strongly foliated outcrops east of the Big River. In the Widgeon Pluton, the foliation dips more gently to the west. Higher strains are apparent at the microscopic scale in later sub-millimetre wide shear zones that include minor fine-grained sericite, more intense sub-grain development in adjacent feldspar grains, and ribbon quartz.

Some dikes derived from the Big Pluton are superficially similar to centimetre-wide leucogranite veins localised in narrow centimetre-wide ductile shears, crenulation surfaces, and boudin necks which cut the adjacent Fanny Bay Group. These narrow leucosome veins are inferred to have been generated during partial melting of the metasedimentary rocks. However, where dikes, folds, and leucosomes occur in the same outcrop (e.g., B45/366474), the dikes cut the fold axial planes whereas the leucosomes are parallel to the axial planes, suggesting that emplacement of the Big Pluton postdates peak metamorphism, partial melting, and development of most if not all outcrop-scale penetrative structures in the Fanny Bay Group.

Both the Big and Widgeon Plutons intrude the quartzofeldspathic Fanny Bay Group to their west and the psammite, amphibolite, and calc-silicate-dominated Cameron Group to their east (Fig. 2). The Widgeon Pluton in particular cuts quartzite-bearing Fanny Bay Group rocks to the west and contains rafts of Cameron Group psammitic and calcsilicate rocks in the east. In Big River, where these units are not separated by plutons, their contact is a major fault. These two groups have been correlated with the Buller and Takaka Terranes, respectively, in northwest Nelson (Ward 1986; Powell \& Kimbrough 1987; Cooper 1989; Powell 2001), where they are separated by the Anatoki Fault. This correlation implies that, in Fiordland, these distinct metasedimentary units have been in close proximity since the early Carboniferous.

Dikes of fine to medium-grained massive biotite granodiorite and leucogranite cut c. $10 \%$ of the outcrops in parts of the Big Pluton. Although many are likely derived from adjacent younger plutons, at least one of these dikes has 


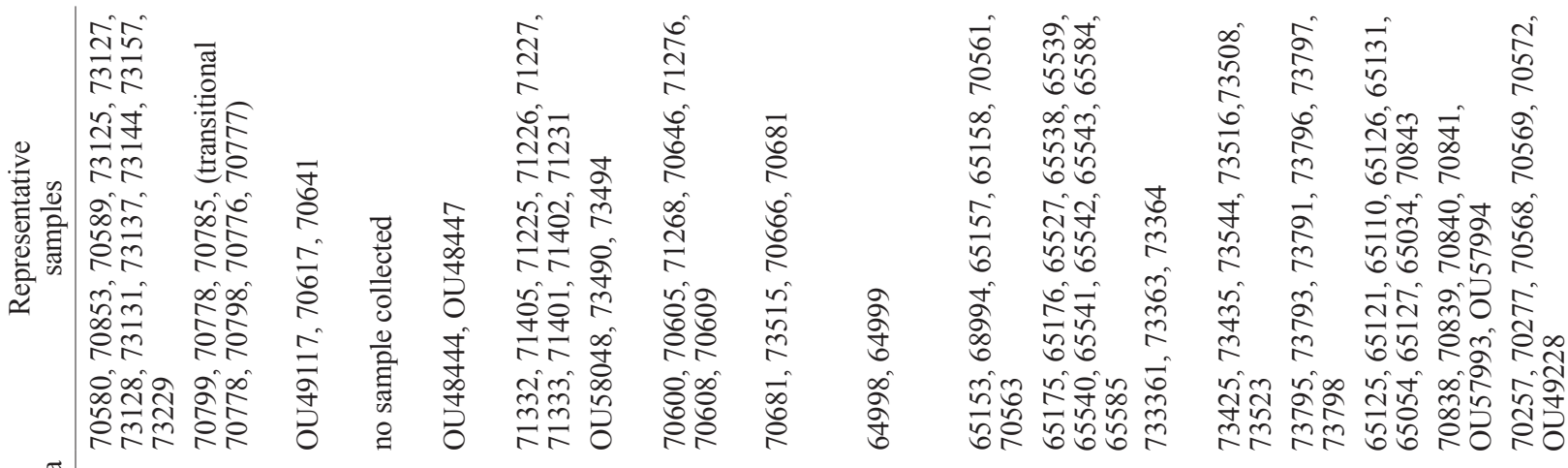

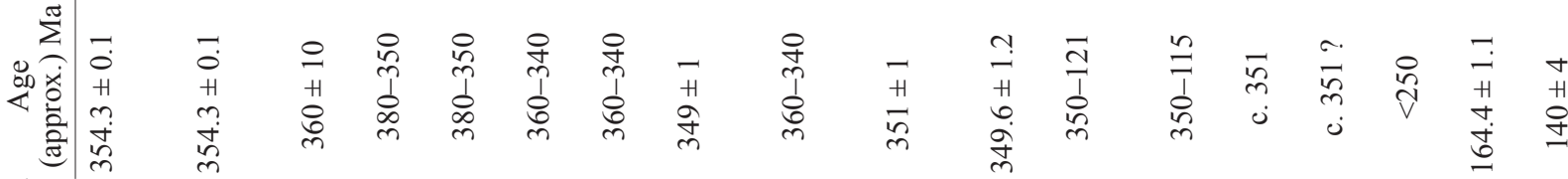

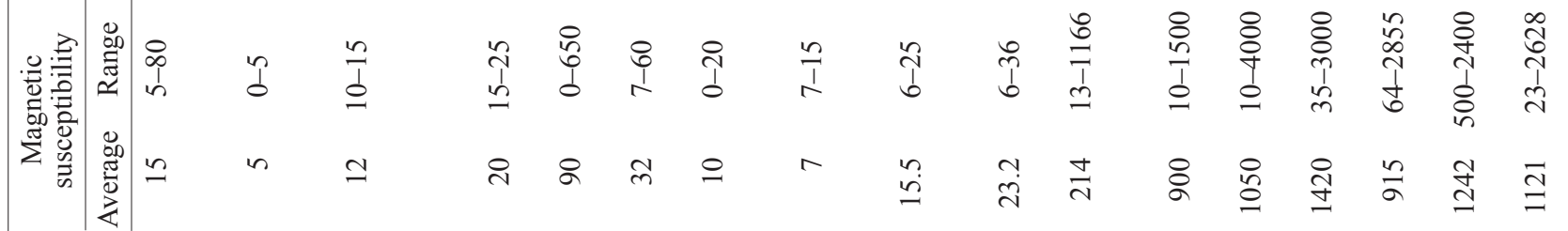

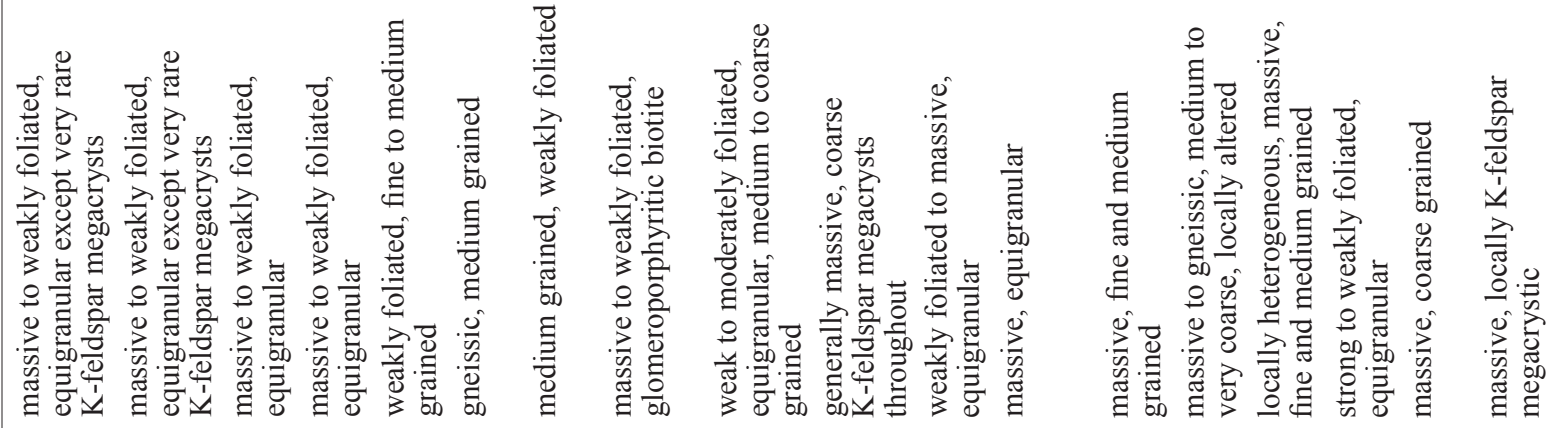

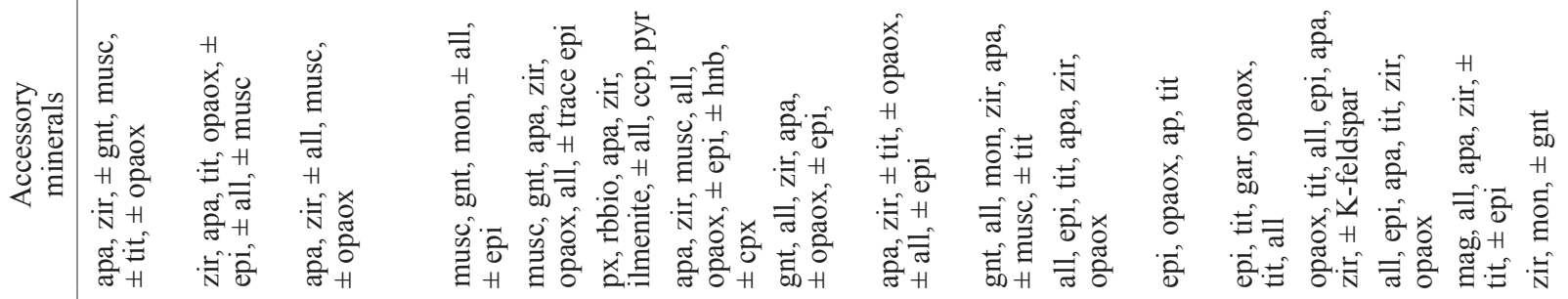

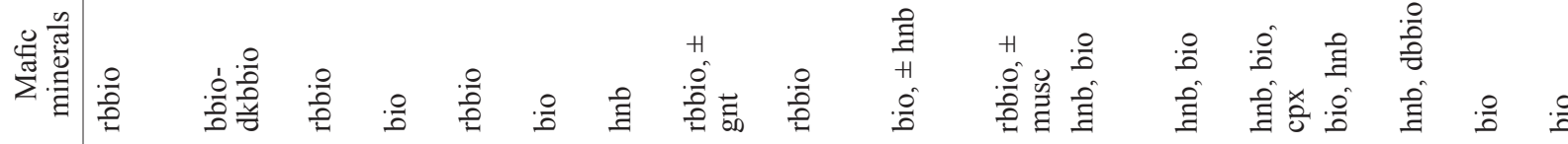

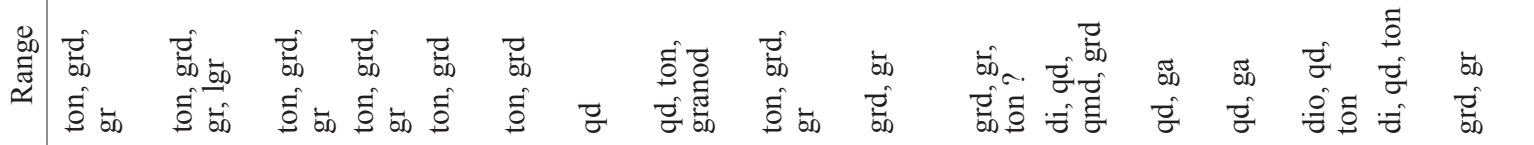

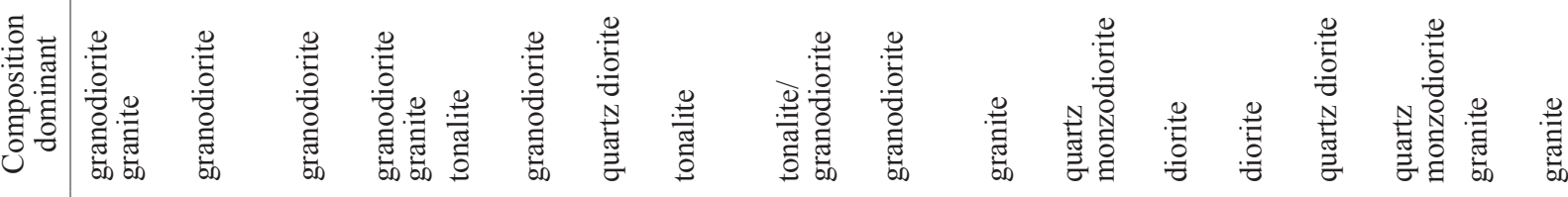


Allibone et al.-Plutonic rocks, SW Fiordland

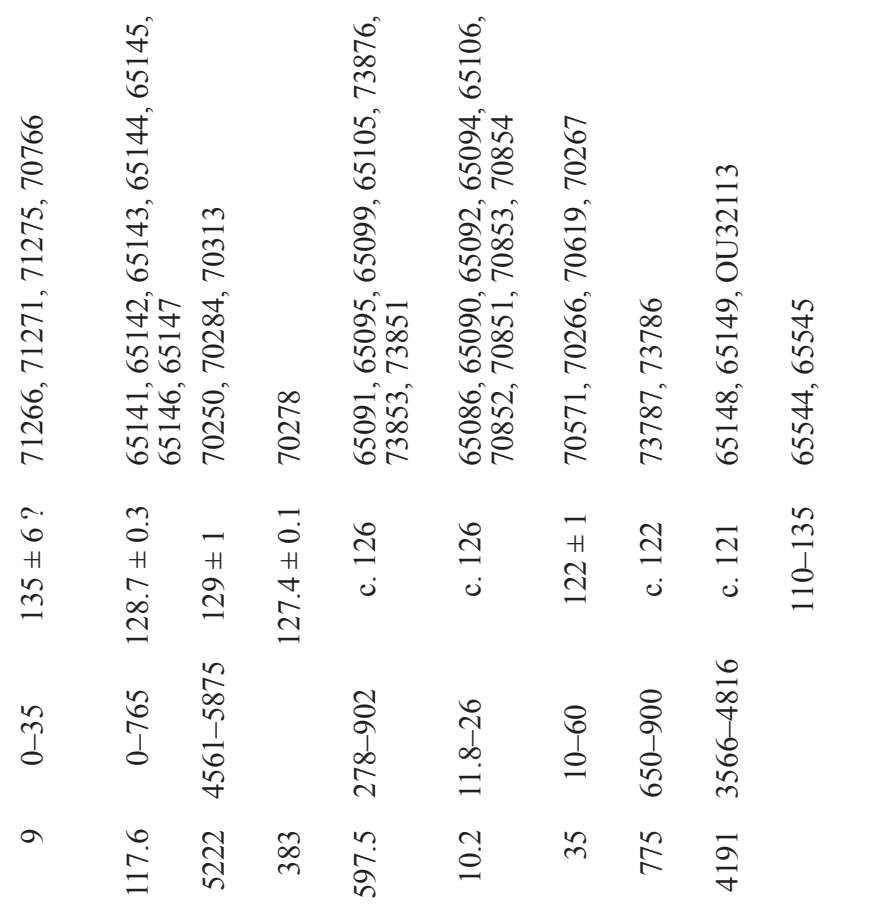

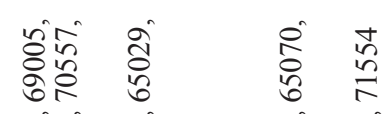

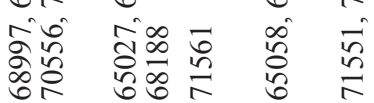

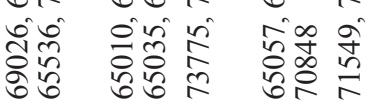

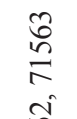

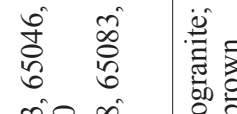

章部

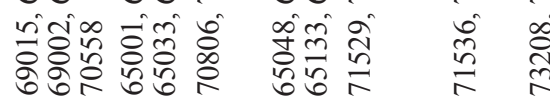

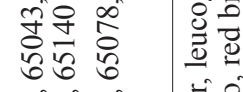

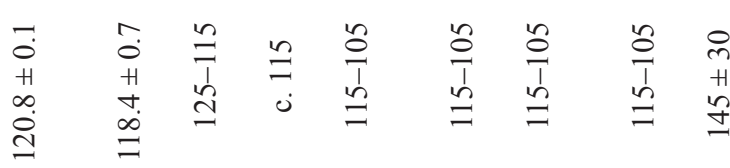

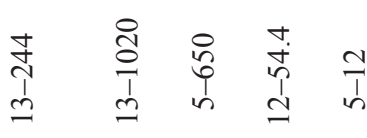

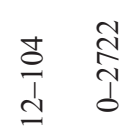

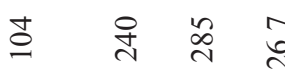

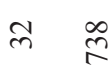

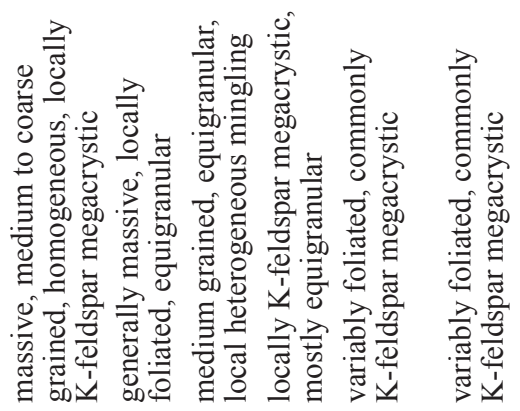

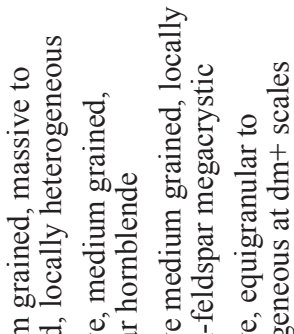

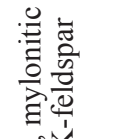

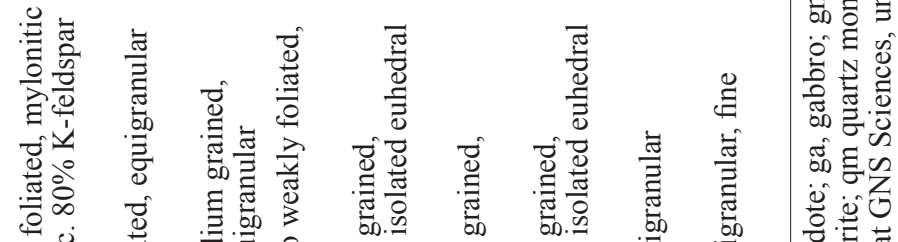

(1)

芸产要

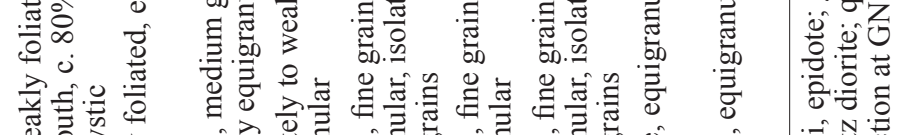

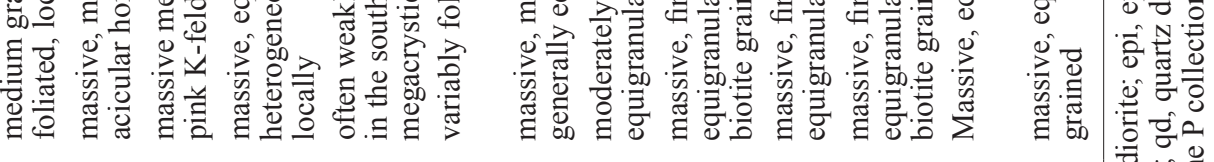

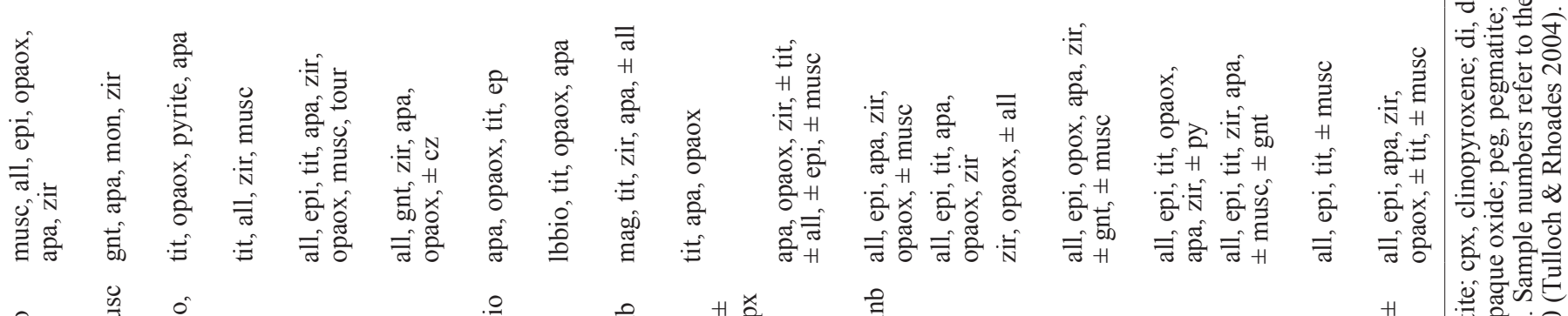

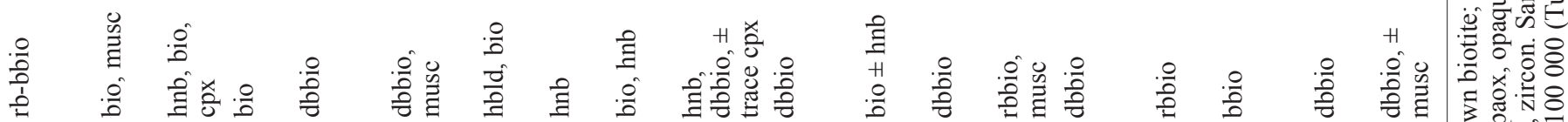

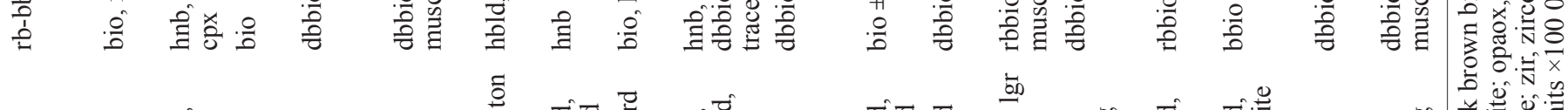

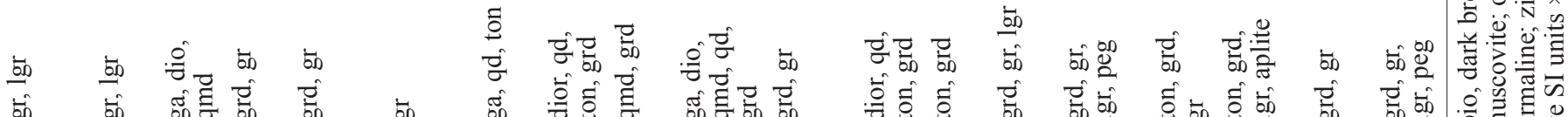

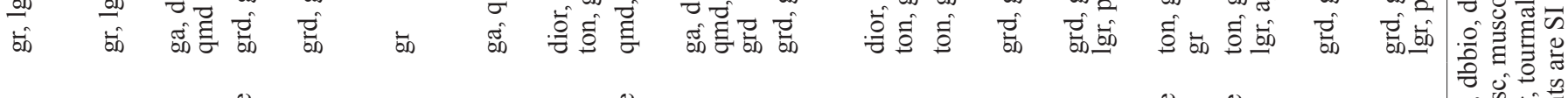

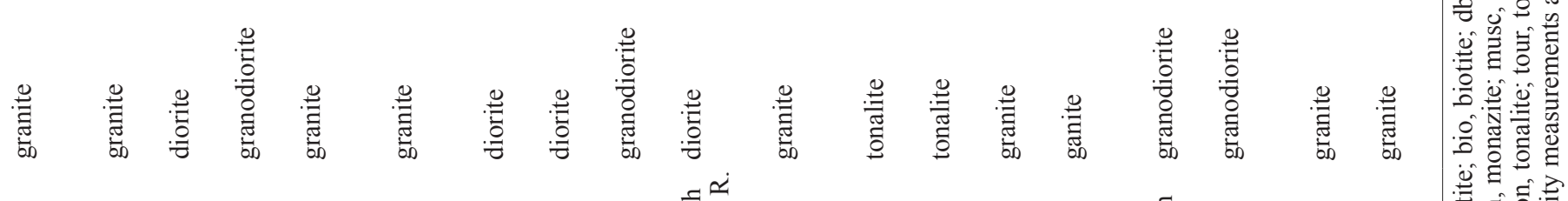

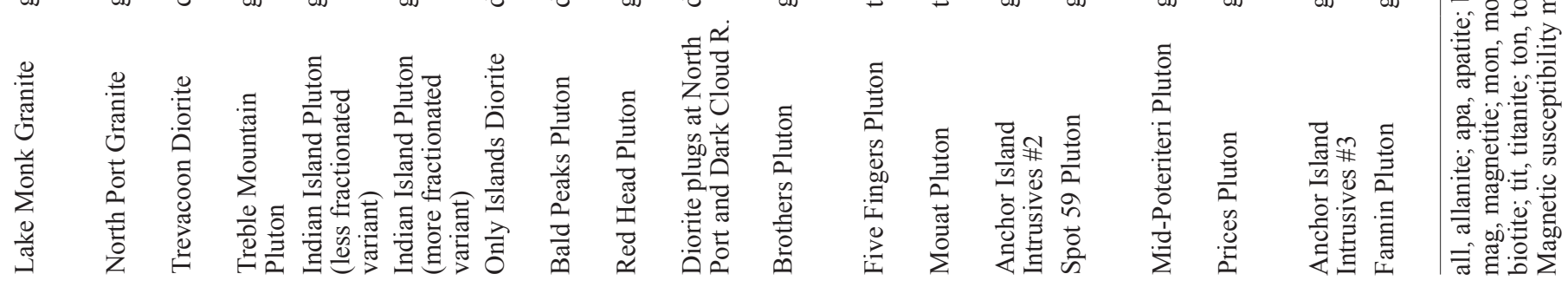


a similar mineralogy to the Big Pluton itself and may simply be a finer grained late fraction that intruded the main body of the pluton (P70795).

\section{Houseroof and Lake 773 Plutons, and Thundercleft Quartz Diorite}

Houseroof Pluton (new name) is an elongate NNE-trending intrusion c. $45 \times 5 \mathrm{~km}$ in size in the north-central Cameron Mountains (Fig. 2). It is named from extensive exposures near the centre of the pluton at Houseroof Hill, the nominated type locality. A swarm of sills and dikes within calc-silicate rocks in the vicinity of Tine Peak marks the northern end of the pluton and was mapped as Tine Peak Tonalite by Powell (2006). (Tine Peak, at B45/481689 is an informal name (Powell 2006), not recognised by the New Zealand Geographic Board). U-Pb monazite TIMS dating indicates an emplacement age of c. 349 Ma. Lake 773 Pluton (new name) forms another narrow, elongate, NNE-trending pluton c. $25 \times 4 \mathrm{~km}$ in size in the Cameron Mountains west and north of Lake Poteriteri (Fig. 2). It is centred on the ridge between Lakes Mouat and Kakapo, near an un-named lake at grid ref. B45/472535 at $773 \mathrm{~m}$ a.s.1. (hence the name). A 2-3 $\mathrm{km}$ wide belt of Cameron Group metasedimentary rocks separates the Lake 773 Pluton from the similar Houseroof Pluton to the west. The Thundercleft Quartz Diorite (Powell 2006) forms a tabular pluton east of the central Long Burn, on and around Mt Thundercleft (an informal name used by Wood \& Grindley (1954) for a peak at B44/448703 southwest from Lake Hay) (Fig. 2). Coeval emplacement of the Houseroof Pluton and Thundercleft Quartz Diorite is implied by the presence of a diffuse mingled contact zone.

Massive to weakly foliated, white, medium to coarse grained quartz diorite and tonalite with minor granodiorite form the Houseroof and Lake 773 Plutons and the Thundercleft Quartz Diorite (Fig. 3). Granite is present in the easternmost part of the Lake 773 Pluton. Equigranular textures dominate, although biotite forms distinct glomeroporphyritic clots throughout much of the Houseroof Pluton. Deep red-brown biotite is the dominant mafic mineral in all three intrusions and is commonly accompanied by accessory garnet. Subordinate quartz diorite in the Houseroof Pluton, and much of the Thundercleft Quartz Diorite, contains hornblende as well as garnet and red-brown biotite (P70609, P73140, P73199, $\mathrm{P} 73490)$. Rare clinopyroxene is also present in both units (OU58048, P70608, P73490). Primary muscovite occurs locally in the Houseroof and Lake 773 Plutons, and rarely in minor tonalitic fractions of the Thundercleft Quartz Diorite (P73494). Ubiquitous coarse apatite and zircon occur in all three plutons and are distinctive petrographic features of these rocks. Coarse euhedral allanite, opaque oxides, monazite, ilmenite, and traces of magmatic epidote are present in many samples.

The Houseroof and Lake 773 Plutons, and the Thundercleft Quartz Diorite, are distinguished from the superficially similar Big, Jeanie, and Widgeon Plutons by their dominant tonalitequartz diorite composition and the presence of hornblende and rare clinopyroxene. Reconnaissance XRF analyses of the Houseroof and Lake 773 Plutons (see below) indicate that both have unusually $\mathrm{Ca}, \mathrm{Fe}$, and $\mathrm{Zr}$ rich compositions notably depleted in alkalis compared with most of the other Paleozoic intrusions of south-central Fiordland. Marble and calc-silicate xenoliths and rafts up to $500 \mathrm{~m}$ long are conspicuous within all three plutons, even though marble is only a rare constituent of the adjacent metamorphic rocks. The dikes and sills which mark the northern end of the Houseroof Pluton are almost entirely confined to calc-silicate host rocks, although foliation-parallel rafts of dioritic rocks occur south of Tine Peak.

Primary igneous, interstitial and subhedral textures are widely preserved in the Houseroof and Lake 773 Plutons. Deformation in these two plutons is generally restricted to development of minor sub-grains and serrated margins on igneous feldspars. Coarse igneous quartz grains are commonly recrystallised to aggregates of finer grains, sometimes with serrated irregular grain boundaries, indicating weak postcrystallisation deformation similar to that observed in other south-central Fiordland Paleozoic plutons. Primary igneous textures are less common in the more strongly deformed Thundercleft Quartz Diorite, suggesting that it may have been emplaced before cessation of penetrative fabric development and peak metamorphism in the adjacent metasedimentary country rocks.

All three intrusions cut the adjacent metasedimentary rocks. Tower Pluton dioritic rocks are crosscut by tonalite dikes derived from the Lake 773 Pluton in the vicinity of Tower Peak. Zones of lit-par-lit intrusions occur along the contact in many places. Rafts of gabbro (P73684), diorite, and gneissic hornblende diorite (e.g., P70662) derived from the Tower Pluton also occur within the Lake 773 Pluton. No crosscutting relationships were observed with the other Paleozoic plutons of south-central Fiordland.

\section{Mt Evans Pluton}

The c. $5 \times 21 \mathrm{~km}$, NNE-trending Mt Evans Pluton (new name) extends from the northern shore of Edwardson Sound to the southern shore of Dusky Sound (Fig. 2). The type locality is the west ridge of Mt Evans at B44/180714. U-Pb zircon dating of a sample from the summit of Mt Evans indicates an emplacement age of c. 349.4 Ma. The pluton comprises incipiently to weakly foliated biotite \pm muscovite granodiorite, granite, and tonalite with accessory apatite and zircon (Table 2; Fig. 3). Red-brown biotite is the dominant mafic mineral. Particularly biotite rich samples commonly contain accessory titanite and only traces of muscovite (e.g., P65157, P65158). Other accessory minerals include garnet, allanite, and monazite. Similar red-brown biotite-muscovite tonalite, which forms a c. $3 \times 2 \mathrm{~km}$ sized raft in the younger Brothers Pluton west of the Newton River (Fig. 2), may originally have been part of the Mt Evans Pluton. Generally, only a few percent of biotite and plagioclase are replaced by chlorite and traces of very fine grained sericite, respectively, although in rare, more altered samples, up to $80 \%$ of biotite and $30 \%$ of plagioclase are replaced by chlorite and sericite, plus minor epidote.

The Mt Evans Pluton intrudes the low-grade Preservation Formation (Fanny Bay Group, Buller Terrane) along its eastern and western margins on the southern side of Dusky Sound, and along the northwest shore of Edwardson Sound (Fig. 2). Dikes and veins of granite, some hybridised to muscovite-rich compositions, occur within the Preservation Formation along the shore of Edwardson Sound. Xenoliths and 10-1000 m scale rafts of hornfelsed Preservation Formation rocks also occur locally throughout the eastern and northern parts of the pluton. Along its western margin south of the Newton River, the Mt Evans Pluton intrudes generally more schistose metasedimentary rocks, similar to those occurring throughout western Dusky Sound and farther north, where they are mapped as Deep Cove Gneiss (Oliver 1980; Gibson 
1982; Ward 1984) (Fig. 2). Mt Evans Pluton therefore appears to stitch these two distinct groups of metasedimentary rocks which are separated by the Dusky Fault in eastern Dusky Sound. Rare decimetre-scale xenoliths of microdiorite and quartz monzodiorite occur within the pluton along the southern shore of Dusky Sound. A narrow ductile shear zone marks the contact with the North Port Granite to the south, while the northern margin may be juxtaposed against Deep Cove Gneiss exposed on Long Island, across the inferred western continuation of the Dusky Fault (Fig. 2).

\section{Newton River Pluton}

Newton River Pluton (new name) crops out along the west coast for c. $5 \mathrm{~km}$ on either side of West Cape (Fig. 2) and c. $4 \mathrm{~km}$ inland up the Newton River. A fault-bounded sliver of Newton River Pluton is exposed on the eastern side of Landing Bay and in creeks to the north. Coastal outcrops at West Cape are nominated as the type locality. The pluton comprises coarse-grained biotite granodiorite and granite with conspicuous coarse, pink, K-feldspar megacrysts (Fig. 3). The accessory mineral assemblage includes titanite, opaque oxide, apatite, allanite, zircon, and rare hornblende. $\mathrm{U}-\mathrm{Pb}$ zircon TIMS data indicate an emplacement age of c. 349 Ma.

Intrusive relationships with other plutonic units were not observed. The Lake Fraser Fault forms the eastern margin of the pluton (Fig. 2). Foliation is best developed in those parts of the Newton River Pluton within c. 100-300 m of the Lake Fraser Fault (e.g., P69029). Later cataclasis overprints this foliation. At Landing Bay, the pluton is strongly foliated and altered with abundant chlorite, calcite, and stringers of subgrain development in quartz, reflecting its close proximity to the Breaker Point Fault (P70353).

\section{GEOCHEMISTRY AND CORRELATION OF PALEOZOIC PLUTONS}

\section{Petrography and previous radiometric dating}

Variations in the petrography of Paleozoic plutons suggest that they form four different groups:

(1) the Big, Jeanie, Widgeon, and Mt Evans Plutons, and Staircase Tonalite, which comprise granodiorite and granite with minor tonalite, and generally contain redbrown biotite \pm muscovite, garnet and sporadic trace titanite, the exception being the eastern part of the Big Pluton which contains dark brown biotite and traces of epidote;

(2) the Houseroof and Lake 773 Plutons and the Thundercleft Quartz Diorite, which largely comprise quartz diorite and tonalite, minor granodiorite and granite with red-brown biotite, garnet \pm hornblende and rare clinopyroxene;

(3) the Newton River Pluton made up of granodiorite and granite, which contains conspicuous coarse, pink, $\mathrm{K}$-feldspar megacrysts, darker brown biotite, and rare traces of hornblende;

(4) The Tower Intrusives, which comprise heterogeneous gabbro, diorite, and minor tonalite.

$\mathrm{U}-\mathrm{Pb}$ zircon and monazite dating indicate emplacement of the Big, Mt Evans, Houseroof, and Newton River Plutons, and the Tower Intrusives, between 348 and $355 \mathrm{Ma}$, indicating that all four groups of plutons are broadly the same age.
In the following discussion, petrography, geochemistry, and age are used to characterise the plutons and compare them with previously described Paleozoic granitoid suites of western New Zealand (Table 2). The radiometric dating implies a correlation between the Paleozoic plutons of southern Fiordland and either the Ridge (S-type) or Tobin (I-type) Suites (Table 1). X-ray fluorescence whole rock analyses undertaken on samples from most of the Paleozoic plutons further clarify their relationship to each other and to established Paleozoic granitoid suites (Tulloch et al. 2003) (Table 3; Fig. 4, 5).

\section{Correlation and petrogenesis of the Big, Widgeon, and Jeanie Plutons, and the Staircase Tonalite}

The peraluminous composition and presence of widespread garnet and muscovite in these plutons, as well as their ages, imply correlation with the S-type Ridge Suite. The majority of analyses of these plutons also plot within previously defined fields for the Ridge Suite (Fig. 4). Samples of these plutons contain greater $\mathrm{Ca}, \mathrm{Na}$ and $\mathrm{Ba}$, less $\mathrm{K}$, lower $\mathrm{Rb} / \mathrm{Sr}$ ratios, and higher $\mathrm{Sr} / \mathrm{Y}$ ratios than S-type plutons previously included in the older Karamea Suite (Fig. 4). The low Rb/Sr and high $\mathrm{Sr} / Y$ ratios of some samples of the Big Pluton extend to values that would generally be associated with I-type rather than S-type granitoids and may reflect derivation of a large part of each pluton from immature volcanogenic and/or related sedimentary rocks rather than old recycled Precambrian material.

The large c. $20 \mathrm{~km}$ east-west extent of the Big Pluton (Fig. 2) is unique in southern Fiordland. The westernmost part of the pluton intrudes the Fanny Bay Group (Buller Terrane), while the eastern part cuts across the Cameron Group (probable Takaka Terrane). This broad range of host rocks, potentially spanning one or more terrane boundaries, implies derivation of the contributing granitoid magmas from a range of sources. Most plutons in the S-type Ridge Suite identified to date are located in the Takaka Terrane (Tulloch et al. 2003). The stronger affinity of the Big Pluton with the Ridge rather than the Karamea Suite is consistent with the location of much of the Big Pluton in host rocks probably correlative with the Takaka Terrane. Intrusion of the Big Pluton would appear to postdate amalgamation of the Buller and Takaka Terranes in southern Fiordland, a conclusion consistent with geologic relationships in northwest Nelson (Jongens 2006).

\section{Correlation and petrogenesis of the Mt Evans Pluton}

The Mt Evans Pluton is petrographically similar to the Big, Jeannie, and Widgeon Plutons. However, analyses show the Mt Evans Pluton tends to be enriched in $\mathrm{K}$ and $\mathrm{Rb}$, and depleted in $\mathrm{Na}$ with lower $\mathrm{Sr} / \mathrm{Y}$ ratios than the other c. 350 Ma granitoid plutons in southern Fiordland. The Mt Evans Pluton instead has a chemistry similar to the older Karamea Suite rather than the Ridge Suite (Fig. 4, 5). This may reflect the Mt Evans Pluton's location in the Buller rather than the Takaka Terrane. In southern Fiordland, Ridge Suite plutonism in the Takaka Terrane at c. 350 Ma appears to have been accompanied by a rejuvenation of S-type Karamea Suitelike plutonism in the adjacent Buller Terrane c. 20 m.y. after emplacement of the main body of Karamea Suite rocks in Nelson (Tulloch et al. 2003). The Mt Evans Pluton could therefore be included in either the Ridge or the Karamea Suite depending on whether age or chemistry are treated as the key defining criteria on which the suites are separated. 


\begin{tabular}{|c|c|c|c|c|c|c|c|c|c|c|c|c|}
\hline N & 芩 & 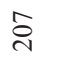 & ఫ্ণ & 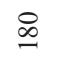 & $\mathscr{\infty}$ & $\bar{\Xi}$ & 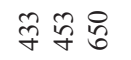 & 光 & $\stackrel{\infty}{\infty}$ & 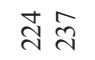 & $\stackrel{m}{a} \cong \stackrel{\infty}{=}$ & 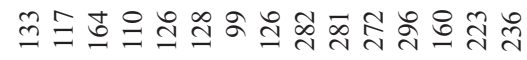 \\
\hline & ช & 0 & in & $\stackrel{\infty}{+}$ & $\stackrel{\circ}{n}$ & $\stackrel{\infty}{n}$ & $\stackrel{n}{n}$ & f & $\overline{0}$ & $\stackrel{2}{\curvearrowright}$ & Iิn के & 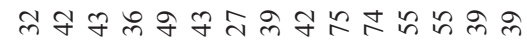 \\
\hline$>$ & নे & $\stackrel{\infty}{+}$ & 字 & 通 & $\stackrel{\infty}{m}$ & ケ & $\stackrel{\infty}{\sim}$ & $m \stackrel{\sim}{m}=$ & $\ddot{n}$ & $\bar{m}$ & 음 & 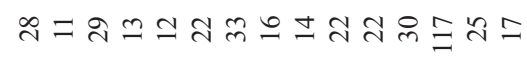 \\
\hline j & in & $\alpha$ & iे & $\stackrel{\infty}{\sim}$ & ते & $\bar{v}$ & $\therefore=$ & $\simeq \infty$ & $\infty$ & ? & $\sigma \vec{v} m^{\infty}$ & 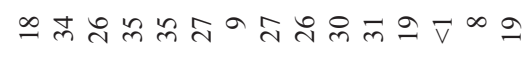 \\
\hline & $\bar{v}$ & $\bar{v}$ & $\vec{v}$ & $\vec{\nabla}$ & $\vec{\nabla}$ & $\checkmark$ & $\vec{v}$ & $\vec{v} \vec{v} \vec{v}$ & $\vec{v}$ & & $-\vec{v}+\vec{v}$ & $\vec{v} n m m \vec{v} n \vec{v} \vec{v} \vec{v} \vec{v} n \vec{v}$ \\
\hline f & $=$ & 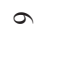 & 6 & $a$ & $=$ & $\because$ & ${ }^{\circ} \vec{v}$ & $\tilde{\imath} \simeq$ & 으 & & $\because \simeq \simeq$ & $\stackrel{\bullet}{v} \bumpeq=\vec{v} \vec{v} \simeq=\vec{\sim} \vec{\sim} \vec{v}^{\infty}$ \\
\hline t & $\tilde{\approx}$ & $\stackrel{\text { ¿ }}{\mathrm{N}}$ & $\stackrel{2}{2}$ & g) & 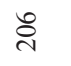 & $\stackrel{\infty}{\infty}$ & ஓั ঞे ஓे & $\mathfrak{m}_{\infty}^{\infty} \sqrt{\infty}$ & $\hat{o}$ & जे ڤे & 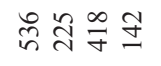 & ల్లి ळ્d \\
\hline & $\vec{v}$ & $\bar{v}$ & $\bar{v}$ & $\overrightarrow{\mathrm{V}}$ & $\simeq$ & 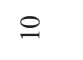 & & $r \circ r$ & $\mathscr{6}$ & 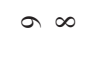 & $6 \sim 6$ & 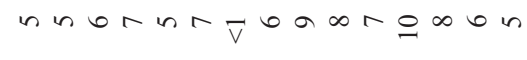 \\
\hline$\frac{f}{a}$ & I & 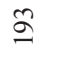 & $\stackrel{\infty}{=}$ & 守 & 寻 & $\stackrel{\infty}{n}$ & & n̊요 & $\stackrel{\infty}{\sim}$ & 药 & 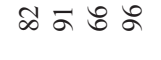 & 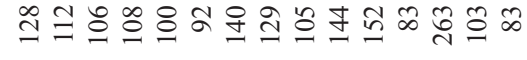 \\
\hline & ส & 7 & $\stackrel{9}{2}$ & 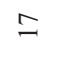 & 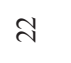 & ले & & $\infty \sim$ & $\Xi$ & & 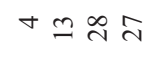 & 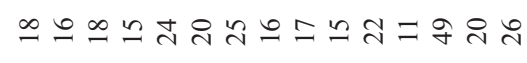 \\
\hline & ת & 4 & in & $r$ & $\infty$ & $\infty$ & & $\omega+$ & $r$ & & $-a$ & $\forall n \sim m \vec{v} m-\infty a \sim b$ \\
\hline$z$ & $\underline{\alpha}$ & $\approx$ & 9 & $\stackrel{\circ}{-}$ & $\stackrel{m}{2}$ & 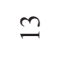 & & $\simeq a$ & $\cong$ & 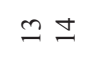 & $\underline{2} \simeq$ & $\circ \circ \simeq \circ==\infty=0 \backsim n$ \\
\hline & $\stackrel{F}{F}$ & 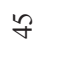 & $\tilde{n}$ & 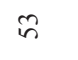 & F & q & & $\hat{n}+\infty$ 周 & $m$ & $\stackrel{q}{q}$ & ले & 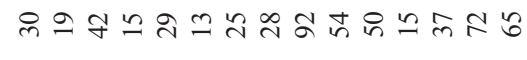 \\
\hline & $\beth$ & 2 & $\stackrel{\infty}{\longrightarrow}$ & $\stackrel{0}{-}$ & $\stackrel{\infty}{-}$ & $\stackrel{\infty}{\longrightarrow}$ & $\stackrel{0}{-}$ & $0 \div 92$ & ণ & $\sim$ & $\dot{v} \pm \cong$ & 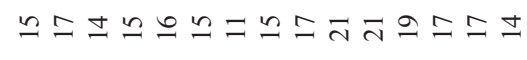 \\
\hline & 6 & + & $\vec{v}$ & N & $\vec{v}$ & $m$ & & $m$ & $n$ & & $n \circ \mathrm{N}$ & $v=+\vec{v} \sim n+\nabla \vec{v}+$ \\
\hline & $=$ & 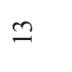 & $m$ & $\underline{0}$ & $\infty$ & 으 & $\vec{v}$ & on & $\vec{\sim}$ & 2 & & $\vec{v}^{n} \vec{v} \vec{v}^{n} \vec{v} \vec{v} \vec{v} \vec{v} \simeq a$ \\
\hline i & 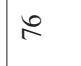 & $\infty$ & $\stackrel{\infty}{n}$ & $\infty$ & $\stackrel{8}{\circ}$ & $\tilde{\sim}$ & n & 崲至 & 2 & $\sqrt{n}$ & లீ: & $\sim n$ \\
\hline & $\hat{2}$ & $\tilde{O}$ & శิ & I & $\hat{8}$ & $\stackrel{\infty}{i}$ & है & 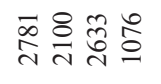 & $\hat{\delta}$ & $\frac{n}{2}$ & oे & 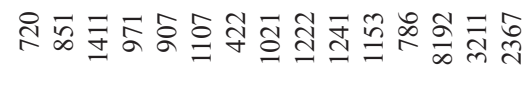 \\
\hline & $r$ & in & in & $\infty$ & $\sim$ & $\vec{v}$ & & $+\triangleleft-\vec{v}$ & - & & $\sim$ in $\bar{v}$ & $\vec{v} \vec{v} \vec{v} \vec{v}-\vec{v} \vec{v} \vec{v} \vec{v} m-c$ \\
\hline & $\begin{array}{l}\infty \\
\infty \\
\dot{\alpha}\end{array}$ & $\stackrel{\vec{a}}{a}$ & $\begin{array}{l}\infty \\
\dot{\alpha}\end{array}$ & $\begin{array}{l}\infty \\
\dot{a}\end{array}$ & ๙ू. & à & & $\begin{array}{l}0 \\
\text { aे } \\
\alpha\end{array}$ & $\vec{a}$ & 5 & त̂े & 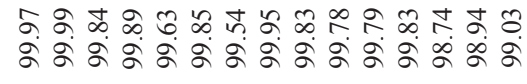 \\
\hline & ڤे & $\stackrel{\circ}{\circ}$ & $\stackrel{0}{0}$ & $\stackrel{\infty}{-\infty}$ & $\stackrel{n}{\mathfrak{0}}$ & สุ & & $\begin{array}{ll}F & n \\
0 & 0 \\
0 & 0 \\
0 & 0\end{array}$ & $\begin{array}{l}\circ \\
\infty \\
0\end{array}$ & s. & 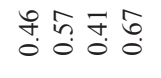 & 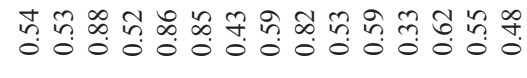 \\
\hline & กิ & 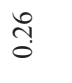 & $\stackrel{\infty}{0}$ & $\stackrel{0}{0}$ & $\stackrel{0}{0}$ & $\stackrel{n}{\circ}$ & & $\stackrel{0}{0}=\frac{T}{0}$ & 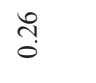 & & : & 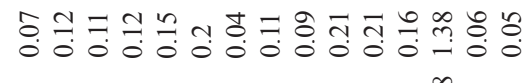 \\
\hline & $\vec{m}$ & 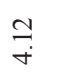 & $\underset{8}{\stackrel{8}{+}}+$ & $\stackrel{\vec{m}}{+}$ & $\stackrel{n}{\rightarrow}$ & $\stackrel{n}{\sim}$ & テ. & it & $\stackrel{+}{-}$ & $i \stackrel{\infty}{m}$ & 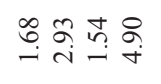 & 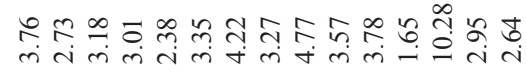 \\
\hline & $\bar{a}$ & $\frac{}{m}$ & $\underset{\dot{r}}{\stackrel{\Delta}{r}}$ & $\stackrel{\grave{i}}{i}$ & $\stackrel{m}{m}$ & $\stackrel{亏}{m}$ & & 홍 & ले & $\begin{array}{ll}+ & 0 \\
i & \infty \\
i & i\end{array}$ & 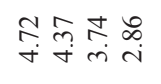 & 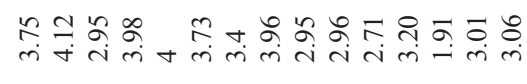 \\
\hline & $\stackrel{\infty}{\infty}$ & $\stackrel{\infty}{\sim}$ & $\infty$ & $\underset{\vec{\sigma}}{\overrightarrow{-}}$ & $\stackrel{?}{\stackrel{n}{i}}$ & $\stackrel{n}{\infty}$ & $=$ & ते گે & $\hat{\sigma}$ & - & ti & ְิ \\
\hline & $\bar{a}$ & $\stackrel{0}{g}$ & $\stackrel{n}{0}$ & $\stackrel{n}{0}$ & $\stackrel{\circ}{\infty}$ & $\stackrel{\infty}{\circ}$ & $\stackrel{n}{m} \underset{i}{\stackrel{1}{-}}$ & 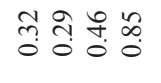 & $\stackrel{\infty}{\stackrel{\infty}{+}}$ & ?. & = & f. \\
\hline & $\stackrel{8}{\circ}$ & $\stackrel{\infty}{\circ}$ & $\stackrel{8}{\circ}$ & $\stackrel{n}{0}$ & $\stackrel{t}{0}$ & $\stackrel{0}{0}$ & 0 & t) & $\stackrel{\infty}{\circ}$ & ?. & 光苛 & 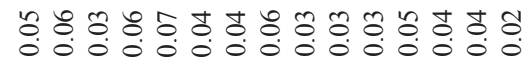 \\
\hline & $\begin{array}{l}\infty \\
\dot{n}\end{array}$ & $\vec{\sigma}$ & $\begin{array}{l}\dot{8} \\
\dot{r}\end{array}$ & $\stackrel{\vec{m}}{\dot{m}}$ & $\stackrel{m}{\sim}$ & $\underset{\sim}{\stackrel{\sim}{n}}$ & $\overrightarrow{0}$ & 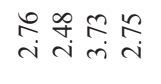 & $\stackrel{\infty}{ָ}$ & ỹ & mेत्̀ & i i \\
\hline & $\begin{array}{l}2 \\
\dot{n}\end{array}$ & $\stackrel{\Re}{\stackrel{\Re}{ \pm}}$ & $\stackrel{\infty}{n}$ & $\begin{array}{l}\stackrel{0}{-} \\
\stackrel{ \pm}{ \pm}\end{array}$ & $\underset{\sim}{\stackrel{\sim}{ \pm}}$ & 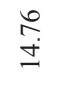 & 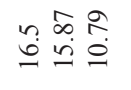 & $\begin{array}{l}\infty \\
\dot{a} \\
\dot{n}\end{array}$ & $\begin{array}{l}\Xi \\
\pm\end{array}$ & F & $\underset{0}{g}:$ & $\stackrel{\infty}{+}$ \\
\hline & $\stackrel{\infty}{0}$ & ڤุ? & $\stackrel{m}{q}$ & $\stackrel{\infty}{\infty}$ & $\stackrel{\text { I }}{6}$ & 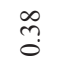 & $\stackrel{0}{i}$ & f. & 8 & $\stackrel{8}{\circ}$ & $\because 2$ & 0 \\
\hline & ầ & $\frac{8}{6}$ & ஓें & ñ? & $\stackrel{+}{\stackrel{1}{\infty}}$ & 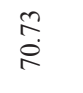 & ț & $\begin{array}{l}k i n \\
\text { in }\end{array}$ & $\frac{\substack{0 \\
0}}{4}$ & $\begin{array}{ll}n & 0 \\
n & n \\
\infty & \infty\end{array}$ & nू. & $\frac{n}{1}$ ? \\
\hline & $\frac{\pi}{\tilde{\delta}}$ & $\frac{m}{\tilde{\delta}}$ & $\begin{array}{l}\stackrel{+}{C} \\
\dot{\delta} \\
\vdots\end{array}$ & $\begin{array}{l}0 \\
\stackrel{\delta}{8} \\
\grave{z}\end{array}$ & 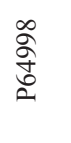 & $\begin{array}{l}\text { के } \\
\stackrel{+}{0} \\
\stackrel{1}{2}\end{array}$ & 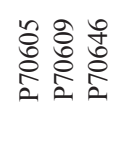 & 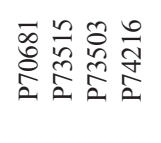 & 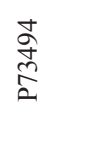 & \begin{tabular}{l}
$\sqrt{n}$ \\
\multirow{2}{0}{} \\
0 \\
$\infty$
\end{tabular} & 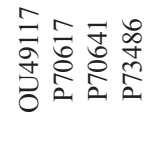 & 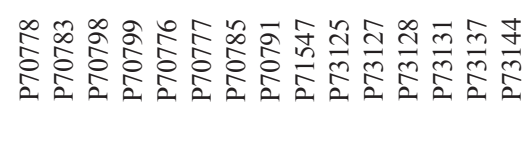 \\
\hline & 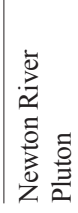 & $\overrightarrow{\vec{z}}$ & 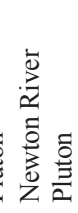 & 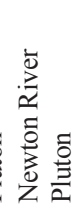 & : & 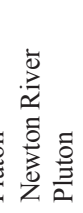 & 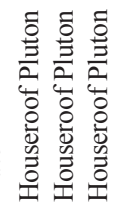 & 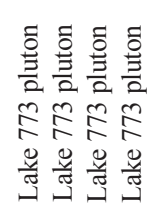 & 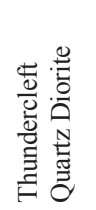 & 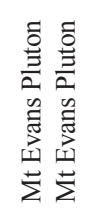 & 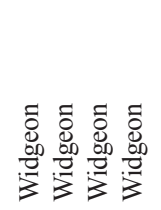 & 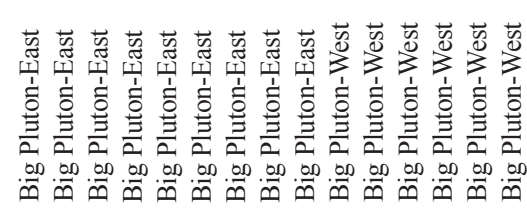 \\
\hline
\end{tabular}




\begin{tabular}{|c|c|c|c|c|c|}
\hline 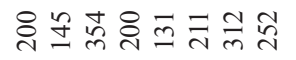 & $\stackrel{\infty}{\infty}$ & $\stackrel{\Xi}{\simeq}$ & हై & $\tilde{\imath}$ & $a$ \\
\hline 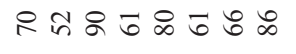 & $\stackrel{\infty}{=}$ & $\stackrel{n}{=}$ & $\stackrel{\Xi}{ٍ}$ & 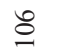 & $\stackrel{\simeq}{\simeq}$ \\
\hline 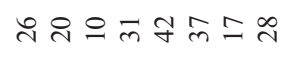 & $F$ & m & 寸 & $N$ & N \\
\hline নি & 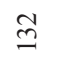 & $\underset{\sim}{\stackrel{J}{\sim}}$ & $\widetilde{\sigma}$ & $\infty$ & 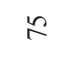 \\
\hline$n m \vec{v} n \vec{v} \vec{v} \vec{v}$ & $\vec{v}$ & $n$ & $\vec{v}$ & $\vec{v}$ & $\vec{v}$ \\
\hline$\simeq \infty \sim \infty \simeq \vec{v}$ & $\vec{v}$ & $\vec{v}$ & $\overrightarrow{\mathrm{v}}$ & $\overrightarrow{\mathrm{v}}$ & $\vec{v}$ \\
\hline テす & $\overrightarrow{\widetilde{\Xi}}$ & ஜ & $\stackrel{8}{8}$ & 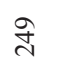 & 6 \\
\hline$n \pm a a, a$ & $\bar{m}$ & 尺े & $\stackrel{\infty}{-}$ & $\stackrel{2}{i}$ & $\simeq$ \\
\hline 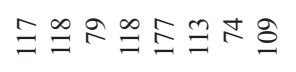 & 8 & ช్ & $\stackrel{ }{\exists}$ & & ナ \\
\hline$\stackrel{\sim}{\circ} \stackrel{\infty}{\sim} \underset{\sim}{\sim}=\simeq$ & 6 & $\simeq$ & $\bar{\sim}$ & $r$ & 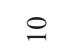 \\
\hline$m n r n m n g$ & n & $r$ & in & สू & $\stackrel{\text { I }}{\mathfrak{T}}$ \\
\hline $\pm コ \cong \backsim 0 a ニ$ & $m$ & $=$ & $\vec{\sim}$ & 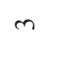 & $\vec{v}$ \\
\hline 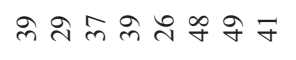 & $\stackrel{n}{n}$ & $\bar{\sim}$ & $\pi$ & & $\vartheta$ \\
\hline ニ & $\hat{\imath}$ & $\bar{\sim}$ & 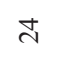 & $\simeq$ & $\stackrel{ }{=}$ \\
\hline Nabo- $0 a \circ$ & ส & \pm & in & 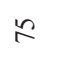 & $\stackrel{\circ}{n}$ \\
\hline$\vec{v}+\simeq \pm \cup \vec{v} \nabla=$ & $\vec{v}$ & $\stackrel{d}{\sim}$ & & : & 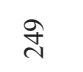 \\
\hline 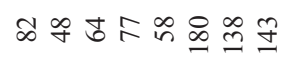 & g & ఫ & t & 2 & 6 \\
\hline 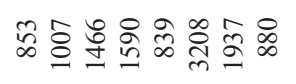 & $\widehat{\cong}$ & F & in & 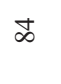 & $\vartheta$ \\
\hline$-\vec{V} \vec{V} \sim N d m i n$ & - & $\vec{v}$ & $\sim$ & $\vec{v}$ & $\vec{v}$ \\
\hline 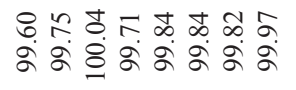 & $\begin{array}{l}\infty \\
\dot{\alpha}\end{array}$ & $\frac{2}{2}$ & $\begin{array}{l}\infty \\
\infty \\
\infty\end{array}$ & $\stackrel{8}{\dot{8}}$ & $\bar{a}$ \\
\hline 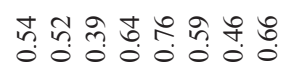 & $\stackrel{+}{\rightarrow}$ & $\stackrel{0}{\mathfrak{s}}$ & 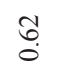 & $\stackrel{+}{\dot{m}}$ & $\underset{i}{\stackrel{+}{+}}$ \\
\hline 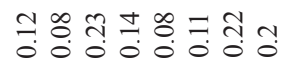 & $\stackrel{n}{6}$ & $\tilde{n}$ & $\stackrel{t}{\stackrel{t}{\circ}}$ & $\stackrel{m}{0}$ & $\stackrel{1}{0}$ \\
\hline 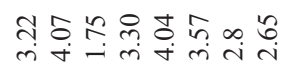 & $\stackrel{\sim}{i}$ & $\stackrel{\rho}{-}$ & $\stackrel{\circ}{i}$ & $\vec{m}$ & $\stackrel{+}{0}$ \\
\hline 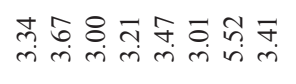 & $\ddot{F}$ & $\bar{\sigma}$ & 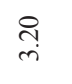 & $\stackrel{m}{0}$ & ले \\
\hline 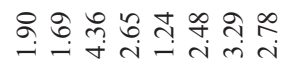 & 7 & $\stackrel{\infty}{\stackrel{\infty}{r}}$ & $\stackrel{\circ}{i n}$ & $\vec{b}$ & $\stackrel{\circ}{r}$ \\
\hline 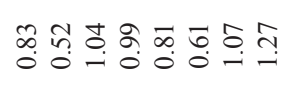 & $\underset{n}{\pi}$ & $\stackrel{\infty}{+}$ & $\stackrel{\text { ga }}{-}$ & $\stackrel{\Xi}{\stackrel{\Xi}{ \pm}}$ & $\stackrel{\infty}{\infty}$ \\
\hline 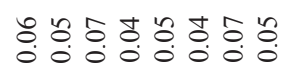 & $\stackrel{7}{\div}$ & $\frac{1}{0}$ & $\stackrel{0}{0}$ & $\frac{9}{0}$ & $\stackrel{\infty}{\circ}$ \\
\hline 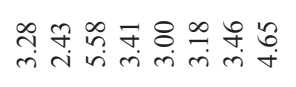 & $\underset{\infty}{\infty}$ & $\stackrel{n}{n}$ & $\stackrel{n}{n}$ & $\stackrel{\dddot{n}}{m}$ & $\stackrel{\Xi}{ \pm}$ \\
\hline 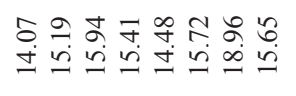 & $\underset{n}{\infty}$ & ๙̊. & $\stackrel{?}{\stackrel{2}{=}}$ & $\stackrel{\infty}{\risingdotseq}$ & 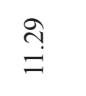 \\
\hline 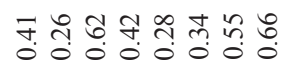 & $\stackrel{9}{=}$ & $\stackrel{\hat{\sigma}}{-}$ & $\stackrel{\Xi}{\Xi}$ & $\stackrel{8}{0}$ & $\stackrel{9}{0}$ \\
\hline 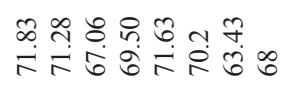 & $\begin{array}{l}\stackrel{\sim}{1} \\
\stackrel{+}{n}\end{array}$ & $\begin{array}{l}\infty \\
\stackrel{\infty}{1} \\
\text { in }\end{array}$ & $\frac{n}{i n}$ & 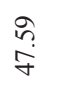 & $\begin{array}{l}\stackrel{\sigma}{.} \\
\dot{\gamma}\end{array}$ \\
\hline 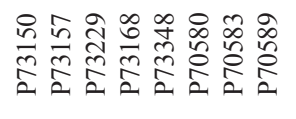 & 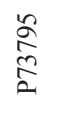 & $\begin{array}{c}\hat{n} \\
\hat{\Sigma}\end{array}$ & 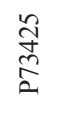 & $\underset{\tilde{n}}{\stackrel{n}{n}}$ & 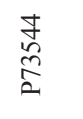 \\
\hline 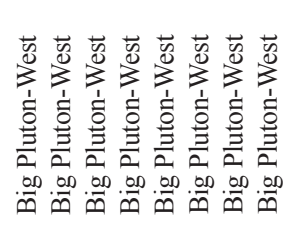 & 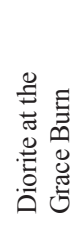 & 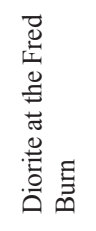 & 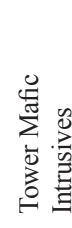 & & 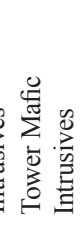 \\
\hline
\end{tabular}

Correlation of Thundercleft Quartz Diorite, Houseroof, and Lake 773 Plutons

The assemblage red-brown biotite, garnet, sporadic hornblende, muscovite, and rare clinopyroxene characteristic of the Houseroof and Lake 773 Plutons, and the Thundercleft Quartz Diorite, is unusual in granitoid rocks. The presence of red-brown biotite and garnet implies correlation with the adjacent Widgeon, Evans, Jeanie, and Big Plutons of the S-type Ridge Suite, some of whose ages are indistinguishable from that of the Houseroof Pluton. However, the presence of widespread hornblende and less common clinopyroxene implies a more calcic composition, reflected in analyses of these three plutons (Fig. 4). Additional chemical features which distinguish these three intrusions from other c. 350 Ma granitoid plutons include their relatively low $\mathrm{K}, \mathrm{Na}$ and $\mathrm{Rb}$ contents, low $\mathrm{Rb} / \mathrm{Sr}$ ratios, high $\mathrm{Ca}, \mathrm{Fe}$, and $\mathrm{Zr}$ contents, higher $\mathrm{Ga} / \mathrm{Al}_{2} \mathrm{O}_{3}$ ratios, and sporadic especially high $\mathrm{Ba}$ contents (Fig. 4, 5). However, Sr/Y ratios are comparable with adjacent c. $350 \mathrm{Ma}$ old plutons that clearly correlate with the Ridge Suite. Some analyses of the western part of the Big Pluton also show hints of the same chemical features as these three plutons, suggesting a petrogenetic link between both groups of c. $350 \mathrm{Ma}$ old plutons (Fig. 4, 5). The high concentration of high field strength elements such as $\mathrm{Zr}$, and low concentrations of alkalies in the Houseroof and Lake 773 Plutons, is consistent with their derivation from source material that had already yielded a granitoid melt enriched in alkali elements at a lower temperature. This implies some similarities between these plutons and peraluminous A-type granitoids (King et al. 1997), although $\mathrm{Ga} / \mathrm{Al}_{2} \mathrm{O}_{3}$ ratios are still less than those typical of A-type granitoids in eastern Australia (Fig. 5) (Collins et al. 1982). Emplacement of more typical S-type plutons at broadly the same time implies two or more phases of melting in the lower crust of south-central Fiordland at c. $350 \mathrm{Ma}$ (see Gollan 2006).

\section{Correlation and petrogenesis of the mafic Tower Intrusives}

The mafic composition and c. $351 \mathrm{Ma}$ age of the Tower Intrusives implies a correlation with the c. 350 Ma old I-type Tobin Suite (Tulloch et al. 2003) and possibly the $349 \pm 5 \mathrm{Ma}$ old Black Giants Anorthosite c. $50 \mathrm{~km}$ to the north (Gibson $\&$ Ireland 1999). The Tower Intrusives are characterised by a LoSY chemistry (Tulloch \& Kimbrough 2003) (Fig. 6). $\mathrm{Sr} / \mathrm{Y}$ ratios decrease from c. 32 to 10 as the $\mathrm{SiO}_{2}$ content increases from c. 41 to $55 \mathrm{wt} \%$ (Fig. 6). $\mathrm{Sr} / \mathrm{Y}$ and $\mathrm{Rb} / \mathrm{Sr}$ ratios are comparable with those of the more mafic plutons analysed to date within the Tobin Suite (Fig. 6) consistent with the c. $350 \mathrm{Ma}$ age of the Tower Intrusives. However, $\mathrm{CaO}$ contents and to a lesser extent $\mathrm{Sr}$ contents are lower than those of previously analysed Tobin Suite plutons, while the $\mathrm{Ga} / \mathrm{Al}_{2} \mathrm{O}_{3}$ ratio and high field strength element content of the most siliceous Tower Pluton sample are both higher than those typical of the Tobin Suite (Fig. 6), hinting at some A-type affinity.

The similar c. $350 \mathrm{Ma}$ age of the Tower Intrusives, Black Giants Anorthosite, and nearby Paleozoic granitoids of southcentral Fiordland indicates broadly coeval ultramafic-mafic and granitoid plutonism at this time. However, trends on chemical variation diagrams defined by the three Tower Intrusives analyses differ markedly from trends defined by granitoid rocks of the same age in south-central Fiordland (Fig. 6). Tower Intrusives samples have higher $\mathrm{Rb} / \mathrm{Sr}$ 

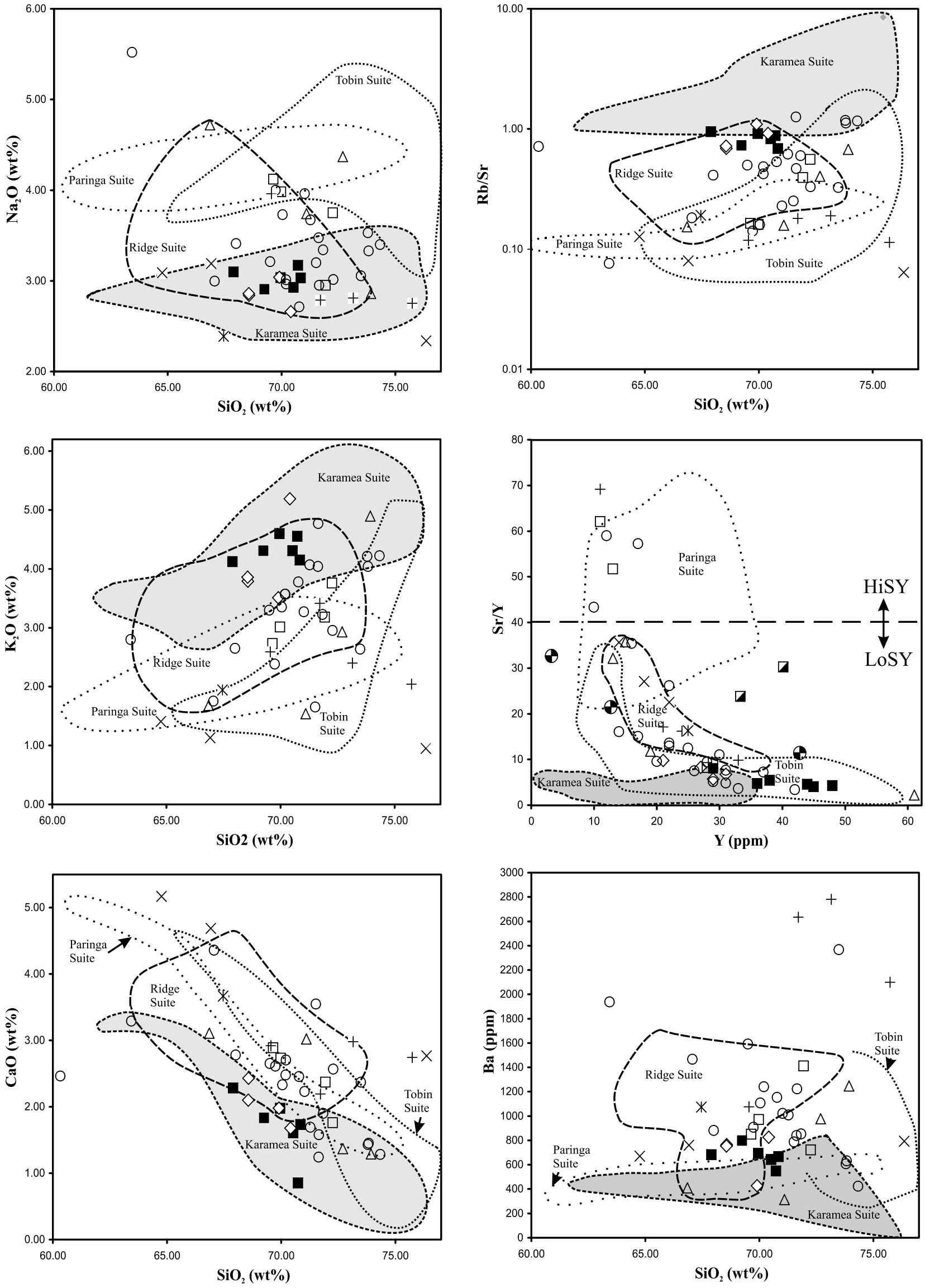

Newton River Pluton $\diamond \mathrm{Mt}$ Evans Pluton $\triangle$ Widgeon Pluton
O Big Pluton West $\square$ Big Pluton East * Thundercleft Quartz Diorite

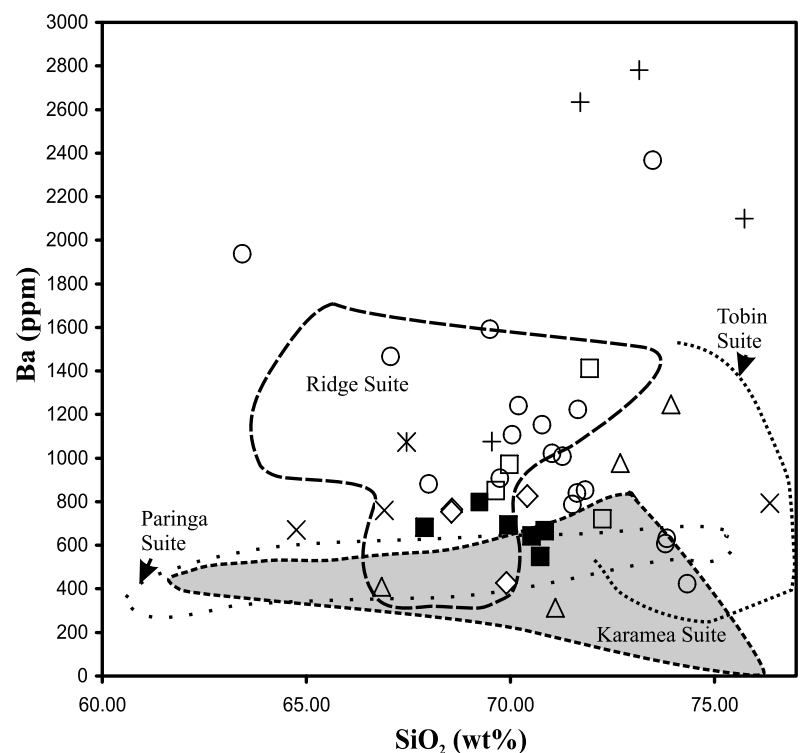

$\times$ Houseroof pluton + Lake 773 Pluton

Tower Intrusives (diorite)

Diorite at the Grace Burn and the mouth of the Fred Burn 

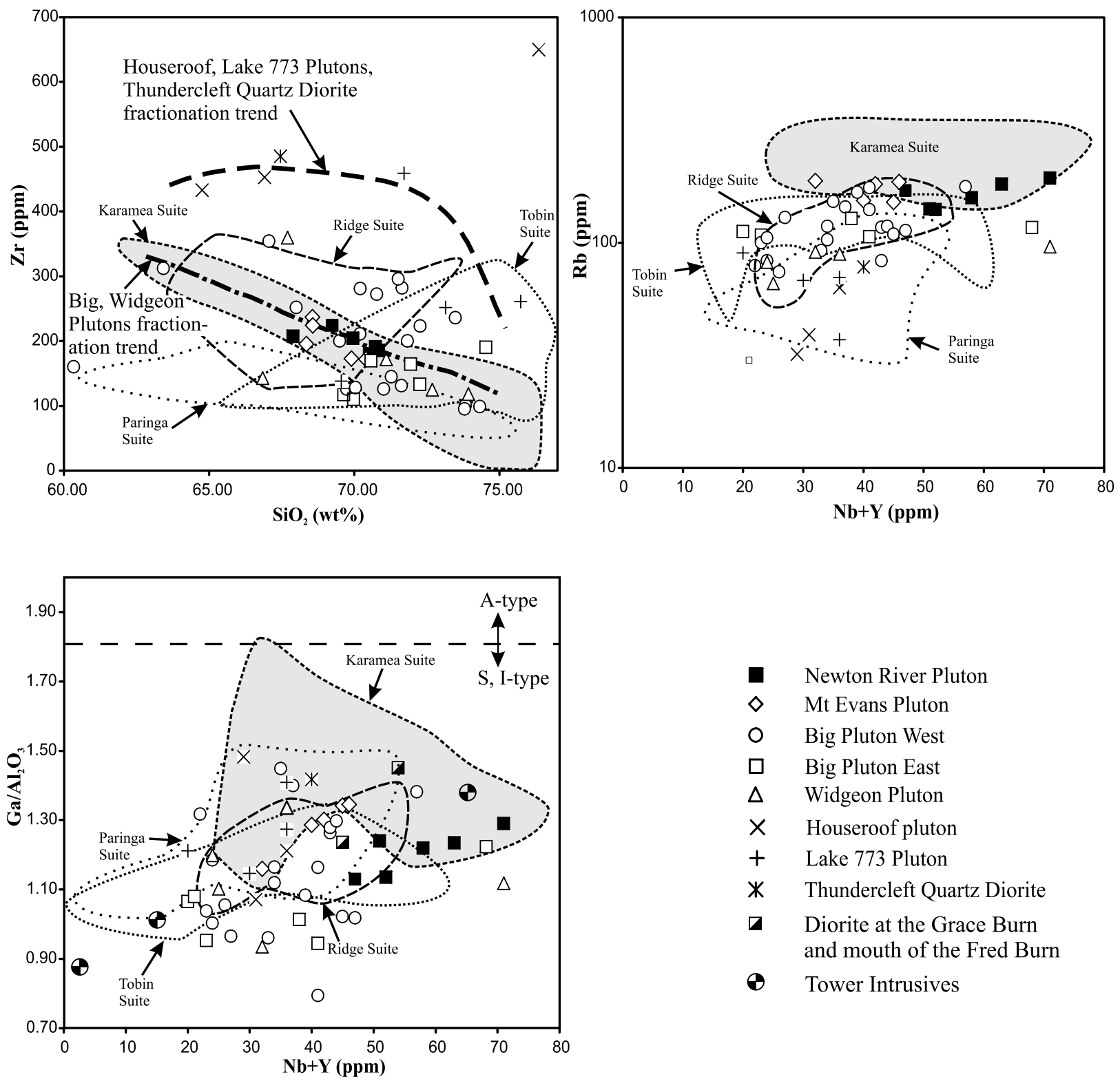

Fig. 5 High field strength element characteristics of Paleozoic plutons in southwest Fiordland. Distinct patterns of high field strength element enrichment characterise the different groups of Paleozoic plutons (see text for discussion).

ratios, $\mathrm{K}_{2} \mathrm{O}$ and $\mathrm{Rb}$ contents, and lower $\mathrm{Ca}$ and $\mathrm{Sr}$ contents at comparable amounts of $\mathrm{SiO}_{2}$ than the granitoid rocks (Fig. 6). $\mathrm{Sr} / \mathrm{Y}$ ratios in the Tower Intrusives decrease as $\mathrm{SiO}_{2}$ content increases, whereas $\mathrm{Sr} / \mathrm{Y}$ ratios in the granitoid rocks vary independently of $\mathrm{SiO}_{2}$ content. The granitoid rocks are therefore neither simple fractionates of a mafic magma similar to the Tower Intrusives, nor simple mixtures of an end member S-type magma derived from melting of Paleozoic metasediments and a mafic magma similar to that which produced the Tower Intrusives. Only the particularly Zr rich composition of the most siliceous sample from the Tower Intrusives suggests a direct link between Tower Intrusives mafic magmatism and the Zr-rich Houseroof and Lake 773 Plutons and the Thundercleft Quartz Diorite (Fig. 6).

Minor diorite bodies at the Grace and Fred Burns in southernmost Fiordland have $\mathrm{K}_{2} \mathrm{O}, \mathrm{CaO}$, and $\mathrm{Rb}$ contents, and $\mathrm{Rb} / \mathrm{Sr}$ and $\mathrm{Sr} / \mathrm{Y}$ ratios similar to those of the Tower Intrusives, rather than other analysed diorite plugs in southwest Fiordland. These bodies may therefore be correlatives of the Tower Intrusives.

Fig.4 Selected variation diagrams illustrating some chemical characteristics of the Paleozoic granitoids in southwest Fiordland. Plutons are compared with compositional fields for the Paringa, Karamea, Tobin, and Ridge Suites as defined in Tulloch et al. (2003) and supplemented by analyses in Allibone (1991); Muir et al. (1996a), Mortimer et al. (1997); Tulloch (1983); Tulloch \& Braithwaite (1986); Tulloch \& Kimbrough (2003), and analyses of Paleozoic granitoids from Stewart Island (Allibone \& Tulloch unpubl. data). See text for discussion. 

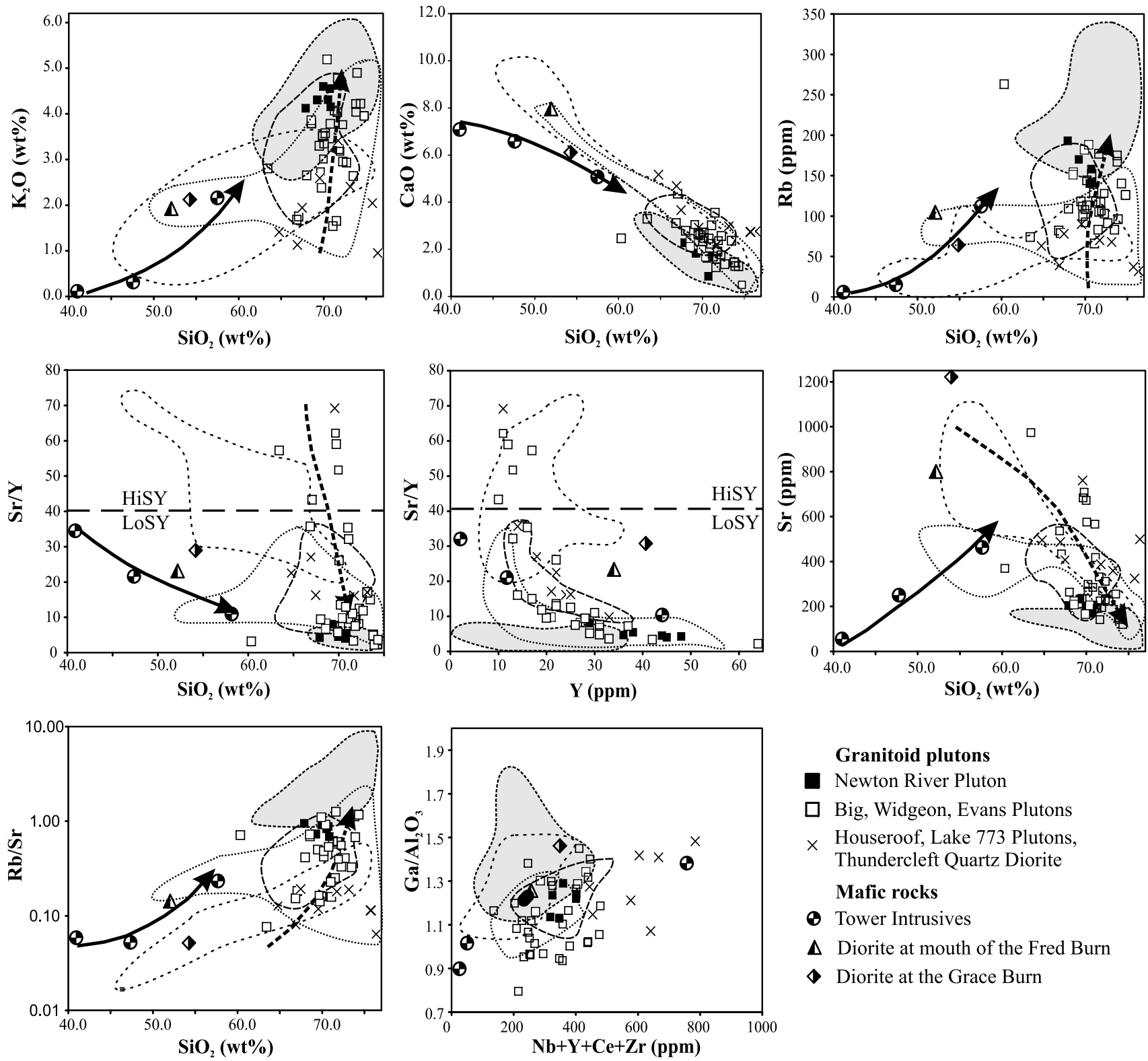

\section{Granitoid plutons}

Newton River Pluton

$\square$ Big, Widgeon, Evans Plutons

$\times$ Houseroof, Lake 773 Plutons, Thundercleft Quartz Diorite

\section{Mafic rocks}

- Tower Intrusives

$\Delta$ Diorite at mouth of the Fred Burn

$\triangleleft$ Diorite at the Grace Burn
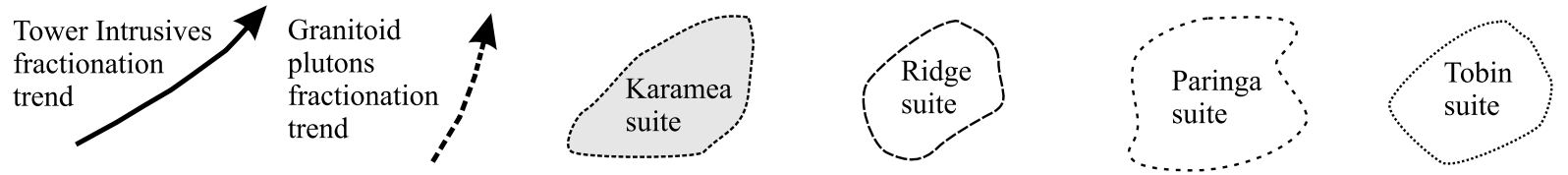

Fig. 6 Comparison of the chemistry of c. 350 Ma Paleozoic dioritic rocks and potential correlative granitoid rocks. Fractionation trends defined by the diorite rocks differ from those defined by granitoid plutons of the same age, implying that the mafic and granitoid intrusions are not directly related.

\section{Correlation and petrogenesis of the Newton River Pluton}

The dark brown colour of biotite and local occurrence of trace amounts of hornblende, titanite, and epidote in the Newton River Pluton imply an I-type rather than an S-type petrogenesis. When combined with the 349 Ma emplacement age, this might indicate a correlation with the c. 349-340 Ma Tobin Suite (Tulloch et al. 2003). However, analyses of the
Newton River Pluton are consistently depleted in $\mathrm{Na}, \mathrm{Sr}$ and $\mathrm{Ca}$, and enriched in $\mathrm{K}, \mathrm{Rb}, \mathrm{Nb}$, and $\mathrm{Y}$ compared with other Paleozoic plutons in southwest Fiordland (Fig. 4, 5). Many aspects of the chemistry of the Newton River Pluton overlap those of the older Karamea Suite (Fig. 4), and support the earlier suggestion that a rejuvenation of Karamea Suitelike plutonism occurred at c. $350 \mathrm{Ma}$, in the Buller Terrane, c. 25-30 Ma after emplacement of Karamea Suite rocks in the Karamea Batholith of northwest Nelson. 
Fig. 7 Compositions of Mesozoic granitoid rocks in southwest Fiordland derived from modal analyses and calculated mesonorms (LeMaitre 1989) plotted on a QAP diagram (Streckeisen 1976). Compositions of both LoSY and HiSY granitoids extend from quartz diorite through tonalite and granodiorite to granite sensu stricto. Mesonorms calculated from XRF analyses are generally richer in K-feldspar than modal analyses of the same units because the mesonorm calculation often does not allocate sufficient $\mathrm{K}_{2} \mathrm{O}$ to biotite. Mesonorms of tonalites therefore tend to plot as granodiorites.

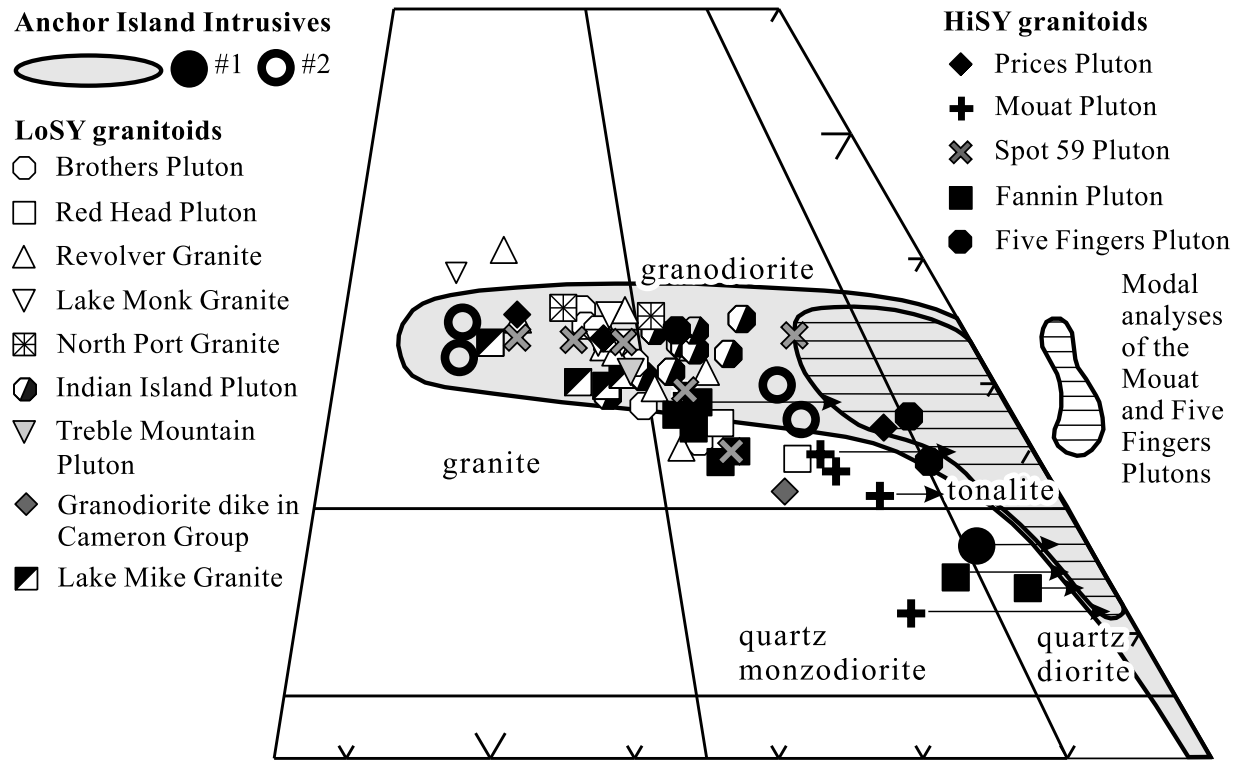

\section{MESOZOIC INTRUSIVE COMPLEXES}

\section{Anchor Island Intrusives}

Anchor Island Intrusives (new name) comprise numerous, relatively small, variably foliated bodies of tonalite, granodiorite, and granite with minor amounts of quartz diorite, diorite, leucogranite, and pegmatite. Many narrow slices and lenses of upper amphibolite facies metasedimentary rocks separate the individual intrusions and comprise c. 10-20\% of the area mapped as Anchor Island Intrusives. Individual intrusions are generally $<500 \mathrm{~m}$ across. These heterogeneous rocks form most of Anchor Island (the type locality, where they are best exposed and from where they are named), parts of many small islands immediately to the east, and the southern shore of Dusky Sound for c. $6 \mathrm{~km}$ west of Cascade Cove (Fig. 2). Isolated slices and lenses of similar orthogneiss and granitoid rocks occur widely in the mountains between Dusky Sound and Chalky Inlet, where they are separated by younger granitoid plutons (Fig. 2). The northern boundary of the unit lies in southwest Resolution Island.

Crosscutting relationships imply that these numerous small intrusions were emplaced during three or more phases of plutonism. The earliest intrusions (Anchor Island Intrusives Generation \#1, Table 2) tend to be the most mafic and strongly foliated, with margins concordant to the foliation and lithologic layering in the adjacent intercalated older schist and paragneiss. Their age is not known. Generation \#1 intrusions comprise hornblende-biotite quartz diorite and tonalite with subordinate diorite (Fig. 7). Accessory minerals include zoned euhedral epidote (some with allanite cores), opaque oxide, titanite, apatite, and zircon (Table 2). Titanite in places rims opaque oxide, suggesting some titanite is a product of alteration (e.g., P65121). Quartz and feldspar form interlocking polygonal grains that generally lack remnants of subhedral and interstitial igneous textures.

Two generations of younger biotite \pm muscovite granitoids (Anchor Island Intrusives generations \#2, \#3; Table 2) cut the more mafic generation \#1 intrusions (Fig. 7). These younger rocks are generally less strongly foliated and often crosscut foliation and lithologic layering in the older intrusions and intercalated schists. Generation \#2 intrusions comprise granite and leucogranite (Fig. 7), with the amount of muscovite exceeding biotite in the most leucocratic bodies (e.g., P65088). $\mathrm{U}-\mathrm{Pb}$ monazite dating indicates an emplacement age of c. 115 Ma (P70848; Tulloch \& Ramezani 2007). Mafic and accessory minerals in generation $\# 2$ rocks include red-brown biotite, \pm opaque oxide, zircon, and rare allanite. Traces of titanite and magmatic epidote occur in those generation \#2 rocks that lack primary muscovite. Subhedral and interstitial primary igneous textures are widely preserved. A gradation from stromatic migmatitic rocks with clear metasedimentary parentage to heterogeneous, xenolith-rich two-mica granite dikes that contain streaky remains of biotite melanosome is present in some outcrops around the northern side of Anchor Island, consistent with a metasedimentary parentage for at least some generation \#2 granites.

Generation \#3 intrusions are sharp-sided discordant dikes of largely unfoliated granodiorite and granite that retain subhedral and interstitial igneous textures. They contain dark brown rather than red-brown biotite, ubiquitous zoned grains of allanite and epidote, and further euhedral magmatic epidote (Table 2). Titanite is commonly present in more mafic samples that contain minimal primary muscovite. Numerous gently dipping pegmatite dikes are the youngest intrusions within the Anchor Island Intrusives. It is not known whether these pegmatites are related to the generation \#3 intrusions or a younger phase of plutonism.

Emplacement of the Anchor Island Intrusives spanned at least the later stages of deformation and metamorphism in the western part of Dusky Sound. Generation \#1 intrusions are the oldest plutonic rocks in the western part of Dusky Sound. At least some generation \#2 granites are likely products of partial melting of intercalated schists. Their c. $115 \mathrm{Ma}$ age indicates that upper amphibolite facies metamorphism in western Dusky Sound occurred during the Early Cretaceous, whereas in south-central Fiordland similar metamorphism predates emplacement of c. 350 Ma old plutons. Generation \#3 granitoids are texturally and petrographically similar to the adjacent Fannin Pluton (see below), suggesting they are marginal dikes of this intrusion. Rare unfoliated, late, 
1-3 m thick gabbro-diorite dikes and relatively small late plugs of coarser grained diorite and heterogeneous quartz monzodiorite (P65069, P65064) within the Anchor Island Intrusives may be related to the nearby Cascade Pluton.

\section{JURASSIC GRANITOID PLUTONS}

\section{Lake Mike Granite}

Lake Mike Granite forms a large, distinctly ovoid pluton and several satellite plugs in the Dark Cloud Range. The type locality is around B45/342685 near Lake Mike. The name Lake Mike Granite is formalised here from mapping and descriptions by Ward (1984), Lee et al. (1991), and this study. The granite is massive, weakly jointed, and forms rugged topography with suppressed vegetation (Lee et al. 1991). It is typically coarse grained, homogeneous, and leucocratic with plagioclase:K-feldspar ratios up to 2:3 (Fig. 7). Accessory minerals include biotite (2-5\%), allanite, zircon, apatite, and magnetite, with sporadic titanite and magmatic epidote concentrated in the more biotite-rich, finer grained parts of the pluton. Alteration is common, with biotite converted to chlorite and titanite, and K-feldspar and plagioclase altered to sericite and prehnite.

Lake Mike Granite intrudes the Preservation and Burnett Formations along its northeast, west, and southern margins, and the Widgeon Pluton (Ward 1984) along its eastern side (Fig. 2). Rare xenoliths include diorite and quartz diorite, possibly derived from the nearby Dolphin Intrusive Complex (Ward 1984). Rare fine-grained aplite dikes occur in the southern parts of the pluton.

$\mathrm{A} \mathrm{U} / \mathrm{Pb}$ age of $168 \pm 8 \mathrm{Ma}$ from the central part of the Lake Mike Granite (OU49118, Ward 1984) is supported by a SHRIMP age of $163.7 \pm 3 \mathrm{Ma}$ (Davids 1999). Recent U-Pb TIMS re-dating of OU49118 indicates an emplacement age of 164.4 $\pm 1.1 \mathrm{Ma}$ (Tulloch \& Ramezani 2007). Geochemical analyses from throughout the Lake Mike Granite are very similar and suggest this age is typical of the pluton. Davids (1999) obtained a Devonian U-Pb zircon (SHRIMP) age of $383 \pm 2 \mathrm{Ma}$ for a sample collected near the pluton margin. This latter sample has a distinct geochemistry and is probably a xenolith derived from an older pluton rather than part of the main body of the Lake Mike Granite.

\section{CRETACEOUS PLUTONS}

\section{North Port, Revolver, and Lake Monk Granites}

The North Port, Revolver, and Lake Monk Granites are described together as they share many petrographic, geochemical, and field characteristics which imply they are closely related. North Port Granite (new name) crops out around the shores of North Port (the type locality), Great Island, and the northernmost of the Passage Islands in Chalky Inlet (Fig. 2). U-Pb zircon dating (P65142; Tulloch \& Ramezani 2007) indicates an emplacement age of c. 128.7 Ma. Revolver Granite (formalised after Powell \& Kimbrough 1987 ) is one of several granitoid plutons originally included in the "Kakapo Granite" but now mapped separately. The name Kakapo Granite is abandoned, but Bishop's (1986) type locality on the eastern shore of Revolver Bay is retained for the Revolver Granite. This $3-10 \mathrm{~km}$ wide by $30 \mathrm{~km}$ long pluton crops out around the shore of Isthmus Sound, Useless Bay, and Revolver Bay, the middle reaches of Long Sound, and on ridges from Long Sound to the south coast (Fig. 2). It includes an unmapped marginal facies referred to as "Long Scarp granodiorite", and a late dike phase referred to as "Upper Blacklock Pluton" by Gollan et al. (2005). Powell \& Kimbrough (1987) reported an Early Cretaceous U-Pb zircon age of c. 130 Ma for a sample collected from Revolver Hill near Long Sound, and referred to this rock informally as "Revolver granite". Subsequent U-Pb zircon and monazite dating indicates an emplacement age of $135 \mathrm{Ma}$ (Tulloch \& Ramezani 2007). Gollan et al. (2005) and Gollan (2006) reported an LA-ICPMS zircon age of $132.4 \pm 1.0 \mathrm{Ma}$. Lake Monk Granite (new name) forms a small (c. $2.5 \times 6 \mathrm{~km}$ ) pluton in the vicinity of Lake Monk between the two branches of Big River (Fig. 2). The type locality is the south side of Lake Monk at B45/434470. The Lake Monk Granite remains undated.

All three plutons comprise homogeneous medium to coarse grained biotite granite (sensu stricto) (Fig. 7) often with a light pink colour. The Revolver Granite also contains minor granodiorite and quartz monzonite (Benson \& Bartrum 1935; Gollan 2006). K-feldspar megacrysts occur widely within the Revolver Granite and more locally within the Lake Monk Granite, whereas the North Port Granite is generally equigranular. Minor biotite is accompanied by variable amounts of muscovite in all three plutons. Muscovite locally exceeds biotite in the North Port and Lake Monk Granites, reflecting their strongly fractionated character. Accessory minerals include zircon and apatite in all three plutons, opaque oxide and traces of allanite and magmatic epidote in the Lake Monk and Revolver Granites, monazite in the Revolver and North Port Granites, and traces of garnet in the particularly leucocratic North Port Granite (Table 2).

The North Port and Revolver Granites intrude the Preservation Formation in Chalky and Preservation Inlets, respectively. A prominent contact metamorphic aureole that includes cordierite-biotite-sillimanite hornfels is developed in Preservation Formation rocks along the western margin of the Revolver Pluton (Benson \& Bartrum 1935). Marginal dikes of the Lake Monk Granite intrude Cameron Group psammitic, amphibolitic, and calc-silicate rocks. Xenoliths of Preservation Formation rocks are common in parts of the North Port Granite, but occur less frequently in the Revolver Granite. The North Port Granite also includes xenoliths of fine to medium grained diorite and mid-upper amphibolite facies garnet-biotite tonalite orthogneiss with accessory titanite, allanite, epidote, and opaque oxide (P71417) on the northwest side of Great Island. The affinity of these tonalitic orthogneiss xenoliths is unknown. The presence of weakly deformed, relatively low-grade Preservation Formation and high-grade orthogneiss xenoliths in the North Port Granite suggests this pluton, like the Mt Evans Pluton, stitches these contrasting rock types which are typical of the Southwest and Western Fiordland regions (Oliver \& Coggon 1979), respectively. Dikes of North Port Granite also intrude a small unnamed gabbro/diorite plug on the northern shore of North Port. The Revolver Granite and similar rocks along the south coast between the Andrew Burn and Green Islets intrude the diorite at the Grace Burn, reducing much of the latter to an intrusion breccia hosted in Revolver Granite (cf. Park 1926). Dikes of fine-grained granite and aplite cut the North Port Granite on 
Great Island, and may be a related late differentiate, since similar dikes were not noted outside the North Port Granite in the surrounding area.

Faults mark some pluton margins. On the northern shore of North Port, a broad zone of strong to intense cataclasis associated with the northeast-striking Breaker Point Fault marks the northern margin of the North Port Granite (Fig. 2). The southern margin of the North Port Granite lies between the Passage Islands, and is also marked by a zone of cataclasis in the adjacent Red Head Pluton, implying it too is a fault contact. The northeast edge of the North Port Granite is marked by a ductile shear zone $(350 / 30 \mathrm{E})$ with an average lineation orientation of 112/34. A north-striking fault partly coincides with the western margin of the Lake Monk Granite south of Lake Monk. Rocks correlated with Revolver Granite are juxtaposed against the Big Pluton across a ductile shear zone in the small bay southeast of the mouth of Big River. Revolver Granite is faulted against diorite across the Last Cove Fault in Long Sound (Benson 1934; Wood 1960), but this fault is a local feature, lacking the regional significance accorded it by Wood (1960), House et al. (2005), and others. Many outcrops of Revolver Granite are extensively altered and red-weathered, especially around Revolver Bay and on the south coast. Brittle fracturing and veining by secondary epidote and chlorite are common in these areas.

\section{Trevaccoon Pluton}

A small pluton of diorite and gabbro that extends from Long Sound across northeast Treble Mountain to above Cunaris Sound (Fig. 2) is named Trevaccoon Pluton, from the nearby Trevaccoon Head in Long Sound. The type locality is the north shore of Long Sound between 2 and $3 \mathrm{~km}$ southwest of Trevaccoon Head.

Trevaccoon Pluton includes medium-grained hornblendebiotite diorite (P70250), sometimes with accessory quartz, fine-grained hornblende-biotite diorite with subordinate clinopyroxene (P70284), and minor gabbro with abundant hornblende and pyroxene largely replaced by epidote and chlorite (P70313). Opaque minerals including pyrite are common accessories. Magma-mingling textures with rounded clasts of finer and coarser grained, more and less mafic diorite in a host of hornblende diorite occur on the Long Sound shoreline. Hornblendite, hornblende-plagioclase pegmatite, and paler leucodiorite dikes also occur within the pluton.

Gollan et al. (2005) report a U-Pb zircon LA-ICPMS age of c. $129 \pm 1$ Ma. Dikes of fine-grained Trevaccoon Pluton intrude Revolver Granite west of Trevaccoon Head. Field relations require the pluton to intrude Preservation Formation above Cunaris Sound but the contact has not been seen. Gabbro and diorite intruding Preservation Formation and Revolver Granite around Last Cove are interpreted to be part of the Trevaccoon Pluton. A hornblende norite described by Benson \& Bartrum (1935) (OU1649, OU1687) from $4.5 \mathrm{~km}$ northeast of Last Cove may be related to the Trevaccoon Diorite, or to Only Islands Diorite in inner Long Sound, since hypersthene has not been recorded from the Trevaccoon Pluton itself.

\section{Treble Mountain Pluton}

Treble Mountain Pluton (new name) crops out over c. $20 \mathrm{~km}^{2}$ of Treble Mountain from where it is named. Outcrops protruding through bush $1 \mathrm{~km}$ northwest of Isthmus Sound at B45/213445 are nominated as the type locality. The pluton was previously included in the Kakapo Granite sensu Wood (1960). U-Pb zircon TIMS dating indicates an emplacement age of c. 127.4 Ma (P70278; Tulloch \& Ramezani 2007) whereas Gollan et al. (2005) reported a U-Pb zircon LAICPMS age of $130 \pm 1 \mathrm{Ma}$.

Treble Mountain Pluton is composed of fine to medium grained biotite granite and granodiorite (Fig. 7), locally containing porphyritic microcline crystals up to $1 \mathrm{~cm}$ long. Abundant sericite, chlorite, and epidote replace primary biotite. Greenish brown biotite or its replacement products constitute c. $5-10 \%$ of the rock. Muscovite is a minor accessory along with rare titanite, zircon, and allanite.

The pluton intrudes Preservation Formation metasediments on its northwest side, with minor sulfide mineralisation along the contact. It intrudes the much coarser grained Revolver Granite above Isthmus Sound and Long Sound, and is inferred to cut mafic rocks of the Trevaccoon Pluton to the east. The pluton is unfoliated except where cut by mylonite and cataclasite zones east of Treble Mountain summit.

\section{Indian Island Pluton}

Indian Island Pluton (new name) forms a $1-4 \mathrm{~km}$ wide, 35-40 km long, NNE-striking intrusion that extends from c. $4 \mathrm{~km}$ south of Dusky Sound to the northeast corner of Resolution Island (Fig. 2). South of Dusky Sound the pluton splits into several narrower dikes that gradually pinch out over c. $4 \mathrm{~km}$. Indian Island in outer Dusky Sound is the type locality. Reconnaissance U-Pb zircon TIMS dating indicates an emplacement age of c. $126 \mathrm{Ma}$ (P70852; Tulloch \& Ramezani 2007).

The Indian Island Pluton comprises variably foliated, often K-feldspar megacrystic granodiorite and granite (Fig. 7). Dark brown biotite is the dominant mafic mineral. Several different accessory mineral assemblages are present within the pluton. In the more biotite-rich and less fractionated parts of the pluton, titanite, allanite, magmatic epidote, opaque oxide, apatite, and zircon accompany biotite with little or no muscovite. More leucocratic parts of the pluton contain widespread muscovite, locally accompanied by garnet and little or no allanite, epidote, or titanite (e.g., P65100, P65102). Tourmaline is a common accessory on Thrum Cap in Dusky Sound. No clear mineralogical zonation was recognised at the scale of the pluton as a whole. Garnet and muscovite-bearing granite occurs around Indian Island, the southern shore of Resolution Island, and in the northern interior of Resolution Island, whereas titanite, epidote, and allanite-rich granodiorite occurs around Thrum Cap in Dusky Sound and the southern and central interior parts of Resolution Island.

Marginal dikes of the Indian Island Pluton cut generation \#1 intrusions of the Anchor Island Intrusives, and xenoliths of the latter also occur in the margins of the Indian Island Pluton. Those parts of the Indian Island Pluton that contain muscovite and garnet are superficially similar to the twomica garnet granite that forms generation \#2 of the Anchor Island Intrusives. However, the two units are distinguished by subtle differences in geochemistry (see below) and the colour of biotite - dark brown in the Indian Island Pluton and red brown in generation \#2 of the Anchor Island Intrusives. Rare, two-mica garnet granite dikes with dark brown biotite that cut the Anchor Island Intrusives south of Lake Rimmer (P65096, P65097), and similar dikes west of Mt Bradshaw (P69017) and near Mt Inaccessible (P70296), may be related to the Indian Island Pluton. 


\section{Only Islands Diorite}

A gabbro-diorite intrusion that extends c. $9 \mathrm{~km}$ from Only Islands in Long Sound southwards across the headwaters of Blacklock Stream was referred to as the Only Islands Diorite by Gollan et al. (2005), and that name is formalised here, with a type locality nominated at the Only Islands. Around the head of Blacklock Stream, Only Islands Diorite comprises massive medium-grained hornblende gabbro and diorite. On the shores of Long Sound, the unit consists of medium to coarse hornblende diorite (P70266), biotite-bearing quartz diorite and tonalite (P70267, 70268, 70264), with accessory apatite, opaques, titanite, and minor epidote. Biotite is often chloritised. Minor hornblende-bearing pegmatites occur as dikes and irregular masses within the pluton. U-Pb zircon (LA-ICPMS) dating of a sample collected south of Long Sound indicates an age of c. $122 \pm 1 \mathrm{Ma}$ (Gollan et al. 2005).

Rafts and xenoliths of metasediment occur within the diorite around Only Islands (cf. Benson \& Bartrum 1935). Southwest of the Only Islands, complex intrusive relationships with the adjacent Widgeon Pluton and Revolver Granite suggest that the Only Islands Diorite as currently mapped may include more than one intrusive phase. Dikes of diorite cut both adjacent granitoid plutons. However, granitoid dikes similar to the two adjacent plutons also cut some of the dioritic rocks, suggesting some diorite is older than both the Revolver Granite (c. 135-145 Ma) and Widgeon Pluton (mid Paleozoic). North of Long Sound in the Dark Cloud Range, possible correlative plugs and dikes of weakly foliated hornblende diorite with minor chloritised biotite, rare quartz, large titanite grains, and accessory apatite, epidote, and abundant opaques (P70619) intrude metasediments and the Widgeon Pluton.

\section{Bald Peaks Pluton}

Fine to medium grained, massive hornblende diorite, quartz diorite, biotite-rich tonalite, and minor granodiorite form the small (c. $2 \times 5 \mathrm{~km}$ ), heterogeneous Bald Peaks Pluton (new name) in the vicinity of Bald Peaks west of the Kiwi Burn (Fig. 2). Bald Peaks form the type locality. Diorite and quartz diorite with conspicuous acicular hornblende grains are interlayered with more siliceous biotite-rich tonalite and traces of granodiorite on a 20-100 m scale throughout the pluton. Particularly mafic samples are dominated by interlocking hornblende and plagioclase grains with minor light brown biotite and accessory titanite, opaque oxide, and apatite (e.g., P73786, P73787). Relict clinopyroxene is present in hornblende cores in P73163. These more mafic rocks lack quartz. Tonalitic and granodioritic rocks contain brown biotite, titanite, allanite, opaque oxide, and sporadic traces of epidote but lack hornblende (e.g., P73160, P73159). Primary igneous euhedral-subhedral textures are preserved with no obvious modification, implying that the pluton is essentially undeformed.

$\mathrm{U}-\mathrm{Pb}$ zircon TIMS dating indicates an age of c. $122 \mathrm{Ma}$ (P73787; Tulloch \& Ramezani 2007), indistinguishable from the age of the Only Islands Diorite several kilometres to the north. The Bald Peaks Pluton forms an ovoid intrusion completely surrounded by the Big Pluton, consistent with the Bald Peaks Pluton being the younger intrusion. A northstriking dike of coarse, massive, pink granite of unknown affinity 200-300 m wide cuts the centre of the Bald Peaks Pluton.

\section{Red Head Pluton}

Red Head Pluton underlies Gulches Head and Red Head (Badger 1973), and extends across Chalky Inlet to Chalky and Passage Islands, and the Balleny Reef (Bishop 1986) (Fig. 2). The unit is revised from the Red Head Member of the Kakapo Granite of Bishop (1986), after Badger (1973), following further mapping and the demise of the Kakapo Granite. Red Head is the nominated type locality. U-Pb zircon TIMS dating indicates an age of 121.1 Ma (P65148; Tulloch \& Ramezani 2007).

Red Head Pluton comprises biotite hornblende granodiorite and subordinate quartz monzodiorite with accessory magnetite, titanite, and magmatic epidote (Badger 1973; Bishop 1986). Pink K-feldspar megacrysts are widely developed at Passage Island, Red Head, and Chalky Island (P70235). On Passage Island, much of the biotite is altered to chlorite, hornblende to actinolite, and plagioclase to fine-grained sericite. Some epidote is likely to have formed during this alteration, but euhedral zoned epidote grains enclosed in biotite are inferred to be of magmatic origin. Carbonate veining and disseminated carbonate grains occur in weakly altered granodiorite on the eastern side of Passage Island (P65149). Although superficially similar to the nearby Newton River Pluton, the Red Head Pluton is distinguished by its more mafic character, distinct geochemistry, and different age.

Numerous xenoliths derived from the Preservation Formation occur within the Red Head Pluton on Passage Island. Xenoliths of fine-grained biotite tonalite (P42684) and diorite (P42681) occur around Gulches Head, where aplite veins and dikes also cut the pluton. Widespread jointing and local cataclasis affects outcrops along the northern shore of Passage Island, indicating a fault contact with the adjacent North Port Granite. Badger (1973) also mapped a faulted contact with Preservation Formation at Gulches Head Peninsula.

\section{Brothers Pluton}

Brothers Pluton (new name) extends from the eastern shore of Cascade Cove in Dusky Sound southwards to the northern shore of Chalky Inlet, making it one of the larger plutons in southwest Fiordland. It underlies much of the Newton River catchment upstream of Lake Fraser, and forms The Brothers hills between the Newton River and Chalky Inlet, from whence it is named. Large slabby outcrops between $\mathrm{Mt}$ Bradshaw and the upper Newton River around B45/120648 are nominated as the type locality. Similar granitoid rocks which form the higher ground northwest of Lake Fraser are also inferred to be part of the Brothers Pluton, separated from the main body by the Lake Fraser Fault. U-Pb TIMS zircon dating indicates an emplacement age of $120.8 \mathrm{Ma}$ (P69005; Tulloch \& Ramezani 2007).

Medium to coarse grained, commonly K-feldspar megacrystic granodiorite and granite with dark brown biotite forms most of the Brothers Pluton (Fig. 7; Table 2). Ubiquitous accessory phases include apatite, opaque oxide and zircon (e.g., P69026, P68997), with monazite, titanite, and allanite (often rimmed by epidote) observed in approximately one-third of specimens (e.g., P69005, P69002). Minor coarse, flaky muscovite that may be of primary magmatic origin is present in some specimens, especially those that lack titanite (e.g., P69026, P70300, P70343).

Rafts and xenoliths of diorite and hornblende-biotite quartz monzodiorite orthogneiss probably derived from generation 
\#1 of the Anchor Island Intrusives, as well as psammitic and pelitic schist derived from the metasedimentary rocks intercalated with the Anchor Island Intrusives, occur widely in the Brothers Pluton. Marginal dikes of the pluton cut generation \#1 of the Anchor Island Intrusives and intercalated schist in streams north of Chalky Inlet. Dikes of Brothers Pluton granodiorite cut the adjacent Cascade Pluton along the shore of Cascade Cove. Dikes of aplite, pegmatite, and biotite-muscovite granite cut less deformed parts of the Brothers Pluton on the western and southern slopes of The Brothers. These aplite and pegmatite dikes may be evolved differentiates of the Brothers Pluton, or related to younger intrusions such as the Fannin Pluton.

The large Lake Fraser Fault forms much of the western boundary of the pluton (Fig. 2). This fault also separates the eastern and western parts of the pluton. A wide mylonitic ductile shear zone is developed in the central part of the pluton south of Lake Fraser. This shear zone is also cut by the Lake Fraser Fault and smaller related brittle faults.

\section{Five Fingers Pluton}

Variably foliated granitoid rocks, here named the Five Fingers Pluton, form c. $40-70 \%$ of Five Fingers Peninsula (Fig. 2). The coastal cliffs facing Dusky Sound northeast from Five Fingers Point form the type locality. The pluton crops out extensively along the remote west coast of Five Fingers Peninsula. Toward the north of the peninsula, narrower bodies of similarly foliated granitoid are interlayered with psammitic and amphibolitic schists at $1-100 \mathrm{~m}$ scales. U-Pb zircon TIMS dating indicates an emplacement age of $118.4 \pm 1.7$ Ma (P65032; Tulloch \& Ramezani 2007).

Five Fingers Pluton includes medium-grained biotite granodiorite with subordinate tonalite, more mafic biotitehornblende quartz monzodiorite, and relatively leucocratic biotite granite (Fig. 7). Remnants of subhedral and interstitial igneous textures have generally survived foliation development. Plagioclase compositions range from $\mathrm{An}_{45}$ to $\mathrm{An}_{25}$. Accessory minerals include conspicuous zoned allanite, in many cases surrounded by corroded euhedral rims of magmatic epidote, apatite, zircon, and opaque oxide (Table 2). Traces of titanite (P65001) and garnet that may be xenocrystic (P65035) occur more rarely. Coarse platy muscovite is common in most samples, generally intergrown with biotite but commonly corroded where in contact with quartzofeldspathic minerals. The coarse grain size and early paragenesis of the muscovite implies a magmatic origin, although the widespread foliation development, and the occurrence of variable amounts of secondary chlorite, fine ragged epidote, and very fine grained sericite after plagioclase indicate that a post-magmatic origin is also feasible.

\section{Mouat Pluton}

Mouat Pluton (new name) was initially mapped as an informal unit ("Ib") by Turnbull \& Uruski (1995), west of Lake Poteriteri. Subsequent mapping indicates that it forms a north-trending, elongate pluton, up to $4 \mathrm{~km}$ wide and $12 \mathrm{~km}$ long, in mountains on the western side of Lake Poteriteri, extending as far east as the lake shore at its widest point (Fig. 2) and as far north as the stream draining Lake Mouat (hence the name). A smaller plug of the same granitoid, 4 $\times 1 \mathrm{~km}$ in size, crops out northeast of Lake Mouat and is interpreted as an apophysis of the Mouat Pluton. A type locality is nominated at Trig 16682 (C45/504409) west of Lake Poteriteri.
The Mouat Pluton comprises white, generally homogeneous, equigranular, fine to medium grained tonalite, and minor granodiorite (Fig. 7). Rare, small, K-feldspar megacrysts with a slight pink colour were noted in one outcrop. More heterogeneous tonalite is present near the western margin of the intrusion where coarser grained but mineralogically indistinguishable blebs occur within the main fine to medium grained phase of the pluton. Later, finer grained granodiorite dikes (e.g., P70812) cut both the coarser blebs and the main phase of the Mouat Pluton. These dikes are either a later phase of the Mouat Pluton or are derived from the adjacent closely related Spot 59 Pluton.

Dark brown biotite is the dominant mafic mineral. Accessory phases include common titanite, magmatic epidote, allanite, opaque oxide, apatite, and zircon (Table 2). Occasional coarse flakes of muscovite intergrown with dark brown biotite (P73527) may also be of magmatic origin. Undulose extinction of quartz is the only common sign of postcrystallisation intra-crystalline deformation. A weak foliation defined by aligned biotite in c. $10 \%$ of outcrops is inferred to reflect weak alignment of biotite during emplacement.

Xenoliths of amphibolite, psammite, and calc-silicate metasediment occur widely, indicating that the Mouat Pluton intrudes adjacent metasedimentary rocks. Rare lenses of coarser biotite-muscovite granodiorite (e.g., P73520) within the Mouat Pluton may be derived from the Paleozoic Big Pluton. Dikes of Mouat Pluton also cut the Big Pluton along the western shore of Lake Poteriteri. The western margin of the pluton is locally separated from the Big Pluton by a brittle fault (Fig. 2).

\section{Spot 59 and Prices Plutons}

Spot 59 Pluton (new name) crops out on both sides of Lake Poteriteri for several kilometres towards the southern end of the lake (Fig. 2). It is named from outcrops near spot height $59 \mathrm{~m}$, the type locality at $\mathrm{C} 45 / 546403$ on the western shoreline of Lake Poteriteri. Spot 59 Pluton extends as far east as the southwestern Princess Mountains, east of Lake Poteriteri. The main body of the pluton extends no more than a few hundred metres west of Lake Poteriteri, although dikes of granodiorite, pegmatite, and aplite probably derived from the Spot 59 Pluton cut the Mouat Pluton as much as $2 \mathrm{~km}$ west of Lake Poteriteri. A small plug, c. $2 \mathrm{~km}$ in diameter, of fine-grained, massive, equigranular granite is well exposed on the coast 1.3-3 km southeast of the Big River mouth (Fig. 2) around Prices Harbour (the type locality), and is named Prices Pluton (new name).

Both Plutons comprise fine-grained granodiorite, granite, leucogranite, and minor leucotonalite (Fig. 7), with isolated distinctly euhedral biotite grains. Pale to dark brown biotite is the dominant mafic mineral. Highly fractionated leucogranites contain muscovite and garnet rather than biotite. Other accessory minerals include allanite, magmatic epidote, titanite, zircon, and apatite. Some outcrops of the Spot 59 Pluton have a banded or streaky appearance, reflecting variations in the grain size of quartz and feldspar, and the proportion of biotite over centimetre to decimetre distances. Marginal dikes in particular may comprise interlayered mixtures of streaky granite, leucogranite, aplite, and pegmatite schlieren.

Xenoliths of Paleozoic metasediment are widespread within both plutons. Marginal dikes of the Spot 59 Pluton cut the foliation in Big Pluton on the southwest shore of Lake Poteriteri, indicating that this pluton was emplaced after 
foliation development in the Big Pluton. The Spot 59 Pluton is also in intrusive contact with the Mid Poteriteri Pluton in the middle reaches of Lake Poteriteri, but their relative ages are not clear. They may be closely related parts of a single composite pluton, although the distinctly different colour and texture of biotite in each (see below) argues against such a relationship. Dikes on the margins of Prices Pluton cut a small body of coarser foliated $\mathrm{K}$-feldspar megacrystic granite of unknown affinity and the southernmost Big Pluton. Xenoliths of the coarse K-feldspar megacrystic granite are also present within Prices Pluton. Varying degrees of brittle cataclasis affect most outcrops of this pluton along the coast, reflecting their close proximity to a major fault(s).

\section{Mid Poteriteri Pluton}

Fine to medium grained, homogeneous, equigranular, white, unfoliated biotite granodiorite and granite crops out for several kilometres along both sides of the middle reaches of Lake Poteriteri and extends eastwards to the Princess Mountains' tops as a series of dikes cutting older Paleozoic metasediments and adjacent granitoids. This intrusion is referred to as the Mid Poteriteri Pluton (new name) (Fig. 2), with a type locality on the eastern shoreline of Lake Poteriteri around C45/545435. Light-brown to red-brown biotite is the dominant mafic mineral, and is accompanied by ubiquitous accessory apatite and zircon, and less common magmatic epidote, allanite, opaque oxide, pyrite, and rare titanite. Biotite grains lack the obvious isolated euhedral shape and dark brown colour of biotite in the otherwise similar adjacent Spot 59 Pluton to the south. Biotite also comprises a slightly greater proportion of the Mid Poteriteri Pluton. The red-brown colour of biotite also distinguishes the Mid Poteriteri Pluton from the nearby Mouat Pluton.

Numerous xenoliths of amphibolite facies metasediment occur throughout much of the Mid Poteriteri Pluton, especially on the western side of Lake Poteriteri. Gradations between xenolith-rich granite, and metasediment intruded by swarms of granitoid dikes, span several 10s to 100 s of metres along the western side of the lake. In one location, granite of the Mid Poteriteri Pluton was observed to grade into pegmatite, suggesting that some of the crosscutting pegmatite dikes may be a late evolved part of the Mid Poteriteri Pluton itself. Other pegmatite dikes may be derived from the adjacent Spot 59 Pluton.

\section{Fannin Pluton}

Fine to medium-grained, equigranular, generally unfoliated, and relatively homogeneous granodiorite and granite (Fig. 7) forms a c. $5 \times 14 \mathrm{~km}$ sized intrusion along the southern shore of Dusky Sound and in hills to the south, which is named the Fannin Pluton (new name) after Fannin Bay (Fig. 2). The coastal section extending eastwards for $4 \mathrm{~km}$ from Fannin Bay is typical of the unit as a whole. U-Pb zircon TIMS dating loosely constrains an emplacement age of c. $145 \pm 30 \mathrm{Ma}$, with a significant inherited component of c. $360 \mathrm{Ma}$ (P69018; Tulloch \& Ramezani 2007).

Granodiorite and granite comprise $>90 \%$ of the pluton with minor pegmatite and leucogranite forming the remainder (Table 2). Dark brown biotite is the dominant mafic mineral and is locally accompanied by muscovite (P64997, P65080, P69018, P66699, P66703). Only rarely does muscovite exceed biotite (e.g., P65078). Accessory minerals include allanite, often rimmed by magmatic epidote, apatite, zircon, opaque oxide, and sporadic titanite (P65083, P65065, P65066).
Titanite and primary muscovite are generally not present in the same specimens.

Strong brittle cataclasis affects the pluton towards the western end of Fannin Bay. The other margins of the pluton are marked by a swarm of dikes, often indistinguishable from the main body of the pluton. These dikes cut generation \#1 and \#2 of the Anchor Island Intrusives, the Indian Island Pluton, and the northwest part of the Brothers Pluton, indicating that the Fannin Pluton is less than c. 122 Ma old. Discordant dikes and plugs of undeformed granite included in generation \#3 of the Anchor Island Intrusives (P65041, P65042, P65046, P65043, P65079, P65078) have the same accessory mineral suite and dark brown biotite as the Fannin Pluton, and may be related. Numerous undeformed, gently dipping pegmatite dikes that cut all other units in the western part of Dusky Sound may also be derived from the Fannin Pluton. Xenoliths of massive diorite and quartz diorite, possibly derived from the Cascade Pluton, and two-mica granite similar to the Indian Island Pluton, occur within the Fannin Pluton along the southern shore of Dusky Sound. The Fannin Pluton is therefore the youngest major intrusion in the western part of Dusky Sound and the surrounding mountains.

\section{GEOCHEMISTRY AND CORRELATION OF CRETACEOUS PLUTONS}

Systematic XRF geochemical analyses were undertaken on specimens from most plutons to clarify their affinities with previously defined suites of Mesozoic granitoids (Tulloch 1983, 1988; Kimbrough et al. 1994; Muir et al. 1998; Waight et al. 1997, 1998) (Table 4). The Jurassic-Cretaceous U-Pb zircon ages of these intrusions imply that they correlate with one or more of the Darran, Rahu, or Separation Point Suites.

High Sr/Y ratios ("HiSY" in the terminology of Tulloch \& Kimbrough 2003), relatively high $\mathrm{Al}$ and $\mathrm{Na}$ contents, and low $\mathrm{Rb} / \mathrm{Sr}$ ratios typical of the Separation Point Suite characterise the Five Fingers, Fannin, Mouat, and Spot 59 Plutons, indicating that these intrusions are likely correlatives of the Separation Point Suite (Fig. 8). The least siliceous sample of Prices Pluton is also strongly HiSY, suggesting that it too is part of the Separation Point Suite. The correlation of these plutons with the Separation Point Suite is consistent with their young field relationships and radiometric ages.

The Mouat, Spot 59, and Prices Plutons bear a striking textural, petrographic, and geochemical similarity to Separation Point Suite plutons on southern and central Stewart Island (Allibone \& Tulloch 1997, 2004; Tulloch \& Allibone unpubl. XRF geochemical data). The hornblendebiotite-epidote-bearing tonalitic-granodioritic Mouat Pluton is mineralogically, texturally, and chemically indistinguishable from the c. 125-116 Ma Escarpment, Easy, Tikotatahi, Doughboy, and Kaninihi Plutons on Stewart Island. The relatively leucocratic biotite \pm muscovite \pm garnet-bearing Fannin, Spot 59, and Prices Plutons are indistinguishable from the c. 116-105 Ma Upper Rakeahua, Lords, Campsite, and Gog Plutons on Stewart Island. These similarities imply a direct correlation between the aforementioned plutons in southwest Fiordland and Stewart Island and are consistent with a general trend to more leucocratic compositions within the Separation Point Suite between c. 125 and 105 Ma.

Lower $\mathrm{Sr} / \mathrm{Y}$ ratios, $\mathrm{Na}$ and $\mathrm{Al}$ contents, and higher $\mathrm{Rb}$ / $\mathrm{Sr}$ ratios characterise the Revolver, Lake Monk and North 

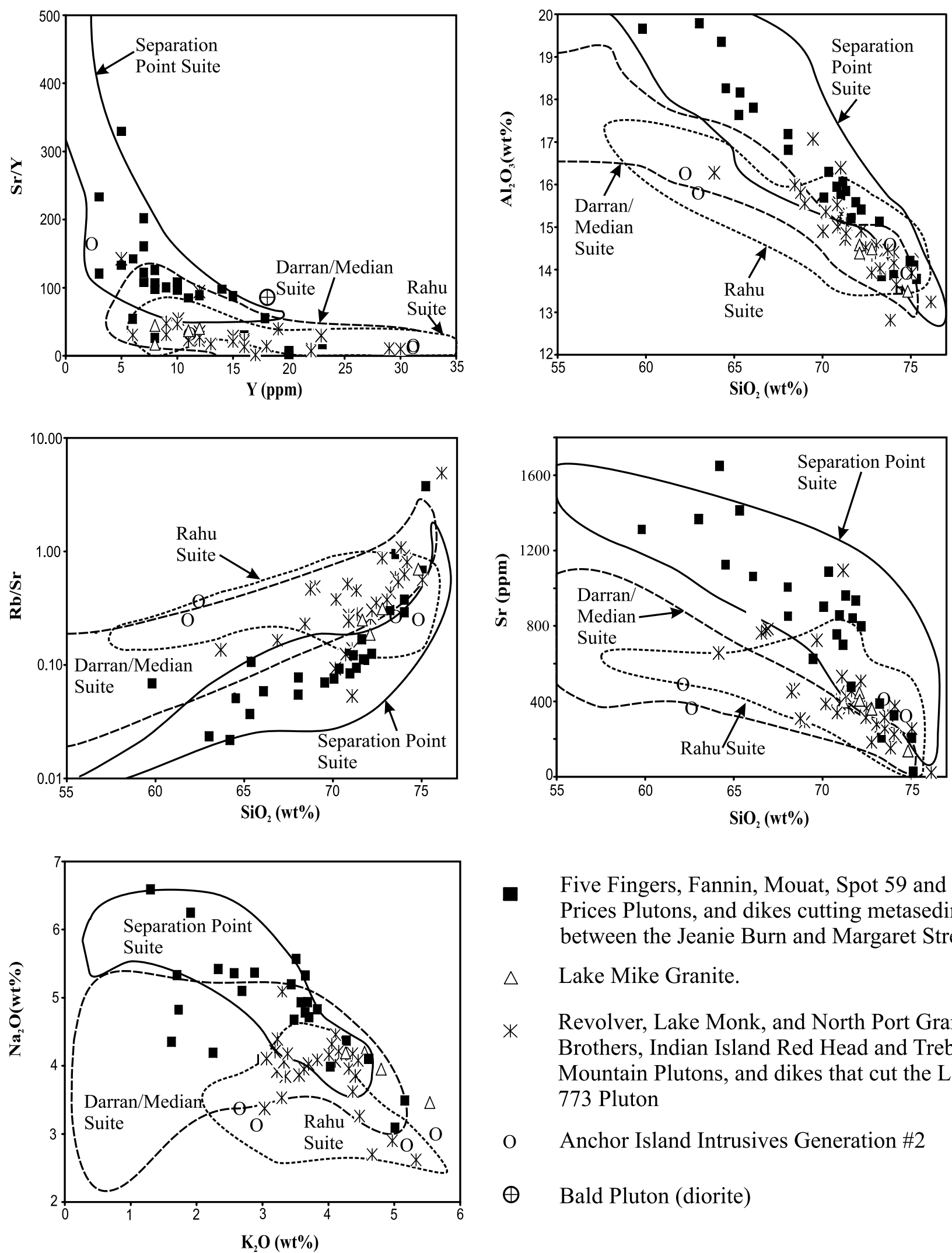

Five Fingers, Fannin, Mouat, Spot 59 and Prices Plutons, and dikes cutting metasediment between the Jeanie Burn and Margaret Stream.

$\triangle \quad$ Lake Mike Granite.

Revolver, Lake Monk, and North Port Granites, Brothers, Indian Island Red Head and Treble Mountain Plutons, and dikes that cut the Lake 773 Pluton

O Anchor Island Intrusives Generation \#2

$\oplus \quad$ Bald Pluton (diorite)

Fig. 8 Selected variation diagrams illustrating some chemical characteristics of Mesozoic granitoids in southwest Fiordland. Compositional fields for the Darran, Separation Point, and Rahu Suites are based on analyses in Tulloch (1983), Tulloch \& Braithwaite (1986), McCulloch et al. (1987), Frewin (1987), Allibone (1991), Blattner (1991), Bishop et al. (1992), Tulloch \& Rabone (1993), Wandres et al. (1998), Muir et al. (1998), Waight et al. (1998), Mortimer et al. (1999), Tulloch \& Kimbrough (2003), and Tulloch \& Allibone (unpubl. data from Stewart Island). 


\begin{tabular}{|c|c|c|c|c|c|c|c|c|c|c|c|}
\hline N & $\stackrel{8}{-}$ & r & & & $\cong$ & 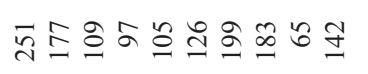 & - & $\checkmark$ & & & \\
\hline ลี & $\beth$ & $\stackrel{\theta}{a}$ & $a$ & 8 & तु & $\stackrel{\circ}{i}$ & 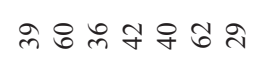 & 5 & 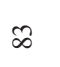 & 年 & \\
\hline$\succ$ & $=$ & $\bar{m}$ & $N$ & & $r \underline{0}$ & 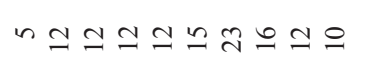 & 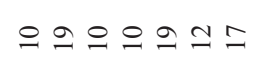 & $a$ & $\infty$ & $\infty=$ & \\
\hline$>$ & $=$ & $\bar{\sigma}$ & $\vec{v}$ & & y $q^{\circ}$ & $\because$ 오ニ & oำ & in & ర్ & & \\
\hline D & $\vec{v}$ & m & $\vec{v}$ & & $\vec{v} \vec{v} \sim$ & $\vec{v} \vec{v} \vec{v} \vec{v} \sim \vec{v} \vec{v} \vec{v} \vec{v} \vec{v}$ & $\vec{v} \vec{v}-\vec{v} \vec{v} \vec{v} \vec{v}$ & m. & $\vec{v}$ & v & \\
\hline 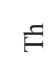 & $\underline{-1}$ & $m$ & m & & $\vec{v} \times 0$ & $\varrho \pm \nabla \vec{v} \vec{v} \vec{v}=a \varrho \pm$ & $a_{\vec{v}}^{\infty} \vec{v} \stackrel{i v}{ } \stackrel{\circ}{0}$ & $r$ & $\vec{v}$ & $\approx \bar{v}$ & \\
\hline$\dot{\omega}$ & $\tilde{\sigma}$ & \& & సे & & 过导导 & 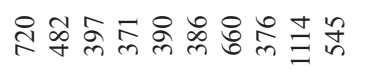 & 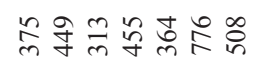 & $\stackrel{+}{\infty}$ & $\stackrel{8}{2}$ & $\cong$ & \\
\hline$\ddot{n}$ & $\vec{v}$ & $\stackrel{2}{2}$ & $\vec{v}$ & & $r$ & $n \sigma_{\vec{v}}+\vec{v} \vec{v} m n-m$ & $\vec{v}^{\infty}+\sin \bar{v}$ & $\infty$ & $m$ & v & \\
\hline थ & $\cong$ & $\hat{\Xi}$ & $\infty$ & & $\approx \infty$ & 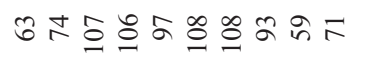 & $\tilde{m} \tilde{m} \tilde{m} \cong \Xi$ & $\stackrel{9}{9}$ & $\simeq$ & 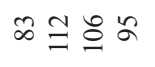 & \\
\hline 2 & q & $\bar{\lambda}$ & $\stackrel{\infty}{+}$ & & $\vec{A}$ & 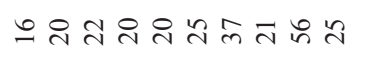 & 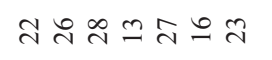 & $a:$ & $\stackrel{m}{=}$ & & \\
\hline $\bar{z}$ & $m$ & $\stackrel{0}{0}$ & 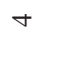 & $\tilde{\sim}$ & $a \infty \nabla$ & o $\vec{v}^{m} \vec{v} \vec{v} \stackrel{\sim}{\sim} \vec{v}^{m-}$ & $\vec{v} r+\vec{v}^{\circ}$ in & $=$ : & $m$ & $\vec{v} \vec{v}$ & \\
\hline z & $\vec{v}$ & $\simeq$ & $\vec{v}$ & 9 & $n r=$ & $r$ 으으요 $\rightarrow a n$ & $\because \simeq r \infty \propto ⿻ \simeq$ & $\simeq$ & 0 & . & \\
\hline$\widetilde{\Xi}$ & $\ddot{m}$ & if & $=$ & & 子气 & $\precsim$ 品ニニュぇ & 뉴워 & $\exists$ & $\stackrel{\Delta}{\sim}$ & & \\
\hline లే & $\simeq$ & $\stackrel{d}{~}$ & 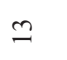 & $y$ & 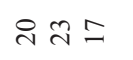 & ニニさ゚ & $\stackrel{\infty}{=}$ & ㅇ & i & & \\
\hline J & n & $\stackrel{m}{=}$ & 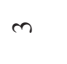 & & $\vec{v}$ & $\sim \stackrel{n}{\sim} \vec{v} \nabla$ & $\vec{v}$ & $N$ & $\mathscr{q}$ & $\vec{v}$ & \\
\hline ¿ & $\vec{v}$ & $m$ & $\vec{v}$ & $g$ & & $\vec{v} \vec{v} \vec{v}$ & $\bar{v}$ & 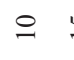 & 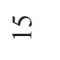 & & \\
\hline ن & 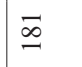 & $\bar{m}$ & 2 & 8 & 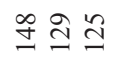 & 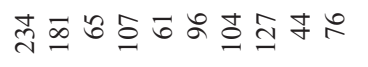 & is & \pm & $\stackrel{\infty}{m}$ & & \\
\hline$\ddot{\infty}$ & ఫे & 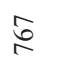 & $\stackrel{\infty}{\stackrel{\infty}{m}}$ & f & 帝点各 & م̂े̆ & $\underset{\Xi}{\Xi} \stackrel{\infty}{=}$ & $\tilde{\infty}$ & 品 & $n$ & \\
\hline \& & s & $\vec{v}$ & $\vec{v}$ & $\bar{v}$ & & $\vec{v} \nabla \sim \vec{v} \vec{v}$ & $m$ & $N$ & $\vec{v}$ & & \\
\hline 声 & $\stackrel{\hat{\alpha}}{\alpha}$ & ๙ু & $\begin{array}{l}\tilde{2} \\
2\end{array}$ & 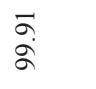 & 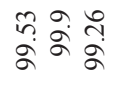 & $\begin{array}{c}\hat{m} \\
\dot{a}\end{array}$ & & $\stackrel{\infty}{\circ}$ & ڤ̆ & & \\
\hline ō & $\sqrt{n}$ & $\stackrel{\infty}{\infty}$ & ?. & $\underset{0}{\infty}$ & f. ?n & if. & $\stackrel{\infty}{n}$ & $\stackrel{0}{\circ}$ & $\stackrel{\infty}{m}$ & & \\
\hline 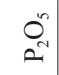 & $\hat{0}$ & $\hat{n}$ & $\stackrel{0}{\circ}$ & $\stackrel{\infty}{\infty}$ & 官 & $\overrightarrow{0}: \stackrel{0}{0} 0$ & $\stackrel{8}{\circ}$ & $\stackrel{t}{n}$ & స̆ & & \\
\hline $\begin{array}{l}0 \\
\sim \\
\sim\end{array}$ & $\frac{1}{n}$ & $\underset{\mathrm{i}}{\mathrm{i}}$ & ธิ & a & & $\bar{m}$ तิ & 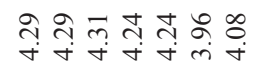 & $\stackrel{尺}{i}$ & ŏ & & \\
\hline$\stackrel{0}{\tilde{\sigma}^{\prime}}$ & $\begin{array}{l}\infty \\
\infty \\
i\end{array}$ & $\stackrel{+}{m}$ & $\stackrel{\infty}{\infty}$ & $\bar{m}$ & & 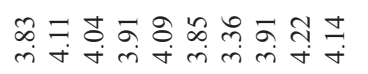 & $=$ & $\stackrel{m}{\rightleftarrows}$ & $\stackrel{m}{\stackrel{m}{m}}$ & $\dot{f}$ & \\
\hline ల̊ & $\stackrel{\tilde{m}}{2}$ & $\stackrel{\infty}{\infty}$ & $\underset{\infty}{\infty}$ & 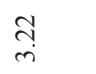 & $\underset{m}{\stackrel{0}{r}} \stackrel{\vec{r}}{i}$ & 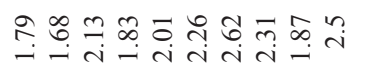 & 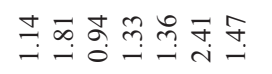 & $\underset{n}{i}$ & $\tilde{\alpha}$ & 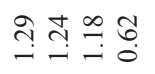 & \\
\hline$\sum_{i}^{\infty}$ & $\sqrt{n}$ & $\stackrel{i}{i}$ & 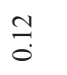 & $=$ & ت̊ & $\vec{m}$ & $\approx$ & $\stackrel{\vec{\bullet}}{-}$ & ते & & \\
\hline$\stackrel{0}{\Sigma}$ & 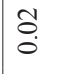 & $\stackrel{0}{\circ}$ & $\begin{array}{l}\overrightarrow{\dot{\sigma}} \\
\dot{\vec{v}}\end{array}$ & : & : & $\overline{0}_{0}^{\circ} \stackrel{0}{0}$ & $\ddot{0}$ & $\stackrel{0}{0}$ & $\overrightarrow{0}$ & & \\
\hline 定 & $\stackrel{\widetilde{2}}{\sim}$ & \&̊م & $\because$ & & & & तె & $\stackrel{\infty}{\infty}$ & $\underset{\infty}{\vec{\infty}}$ & & \\
\hline$\stackrel{0}{\stackrel{1}{\varangle}}$ & $\begin{array}{l}\vec{n} \\
\dot{\Xi}\end{array}$ & $\stackrel{n}{0}$ & \pm & & & 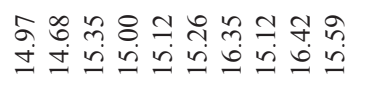 & $\begin{array}{l}\& \\
\dot{ \pm} \\
\dot{y}\end{array}$ & $\begin{array}{l}n \\
n \\
n\end{array}$ & $\begin{array}{l}\infty \\
\stackrel{i}{n} \\
\stackrel{i}{n}\end{array}$ & $\dot{\Xi}$ & \\
\hline$\overbrace{}^{\top}$ & $\overrightarrow{\tilde{o}}$ & ஃ̊. & $\stackrel{\infty}{\circ}$ & $-\pi$ & & & & nิ & $\stackrel{\infty}{\stackrel{\infty}{-}}$ & & \\
\hline $\begin{array}{l}0 \\
\infty\end{array}$ & in & $\hat{n}$ & $\underset{⿱}{\stackrel{i}{+}}$ & ஃ & & 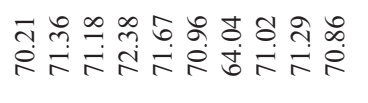 & $g$ & 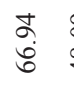 & $\begin{array}{l}\dot{a} \\
\dot{\alpha} \\
\dot{\sigma}\end{array}$ & & \\
\hline 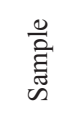 & $\begin{array}{l}\infty \\
\vdots \\
\infty \\
\stackrel{2}{0} \\
2\end{array}$ & $\frac{+}{m}$ & $\frac{0}{0}$ & $\frac{m}{n}$ & 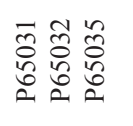 & 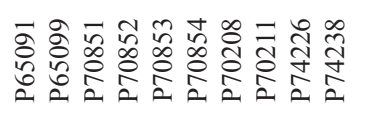 & $\begin{array}{l}10 \\
08 \\
0\end{array}$ & 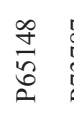 & 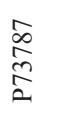 & 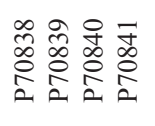 & \\
\hline & 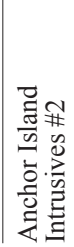 & & & a. & 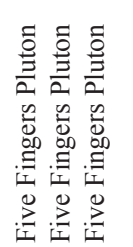 & 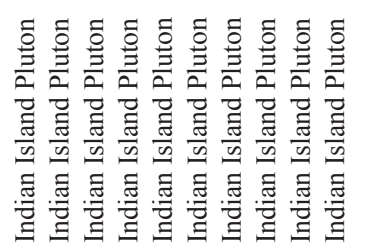 & 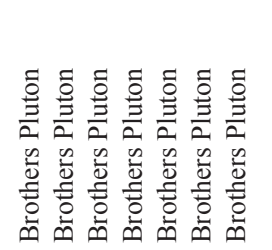 & 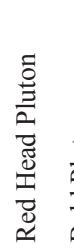 & . & 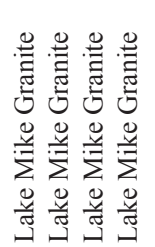 & \\
\hline
\end{tabular}




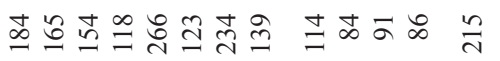

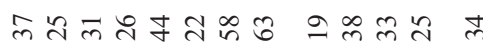

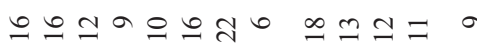

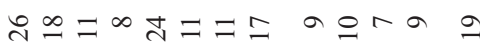
$\vec{v} \vec{v} \vec{v} \vec{v} \vec{v} m+\infty \quad \vec{v}^{n} \vec{v} \quad \vec{v}$

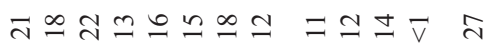

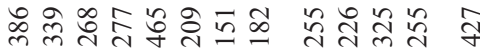
nn $\sim \vec{V}+N \subseteq \infty \quad \infty \vec{v} \vec{v}^{m} \vec{v}$

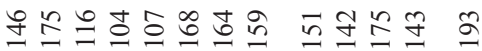

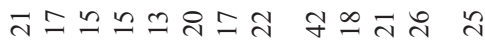
$m \overrightarrow{\mathrm{V}} \mathrm{nmn}-\nabla m \overrightarrow{\mathrm{V}} \overrightarrow{\mathrm{V}} \mathrm{N}$

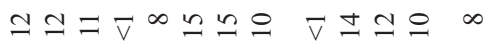

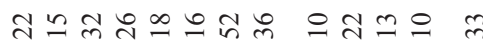

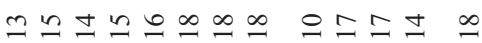
$n \vec{v} n m m \vec{V}-N \quad n \vec{V} \vec{v}$ in $\vec{v} \vec{v} \vec{v} \vec{v} \vec{v}+\nabla \stackrel{\sim}{\vec{v}} \vec{v} \vec{v} \vec{v} \vec{v}$

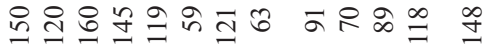

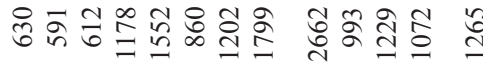
$\vec{v}-m m-c-\vec{v} \vec{v} \vec{v} \vec{v} \vec{v}-$

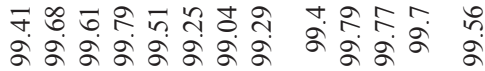

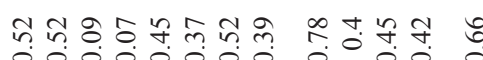

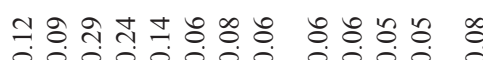
mon

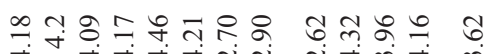
సิ ธิธ兀 귀

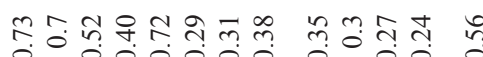
官:

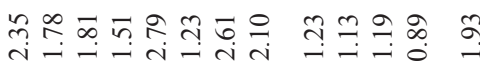

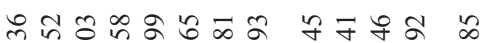

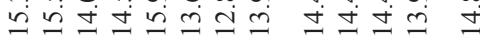
กิ่

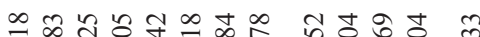
bonmongin

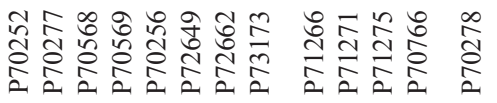

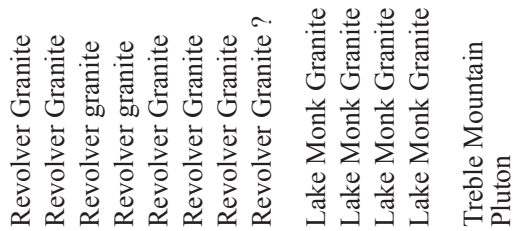

毛守 年

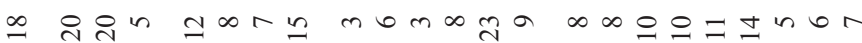
이믈 $\vec{v}+m \vec{v} \sim N \vec{v} b \vec{v} \vec{v} \vec{v} \vec{v} \vec{v} \vec{v} \vec{v} \vec{v} \vec{v} \vec{v} \vec{v} m \vec{v} \vec{v}+$

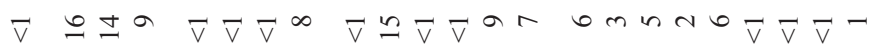

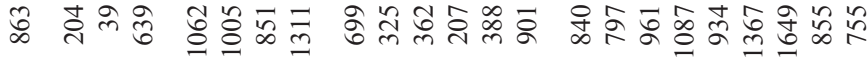

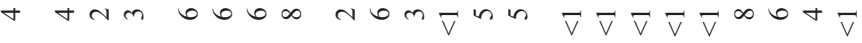

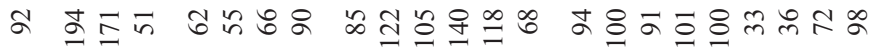

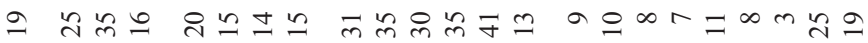

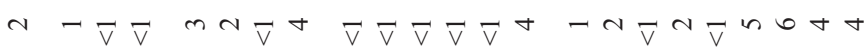

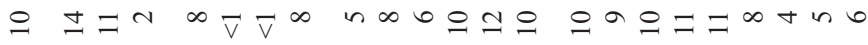

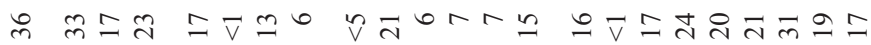

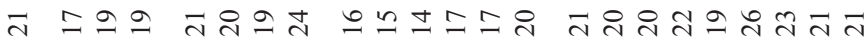
$+N-Z$ nn $n$ in $\vec{v} \vec{v} \vec{v} \vec{v}+-\vec{v} \vec{v} \vec{v} n+\vec{v} \vec{v} \vec{v}-$ $\vec{v}$ nn n $\vec{v} \vec{v} \vec{v} \vec{v} \vec{v} \vec{v} \vec{v} \vec{v}=0$ 二

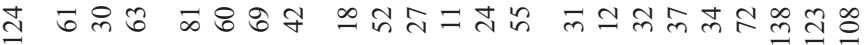

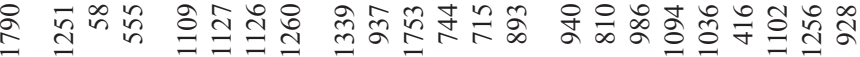

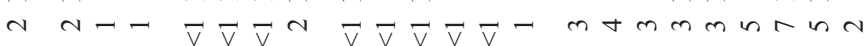

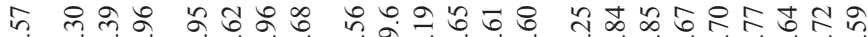
ने इें

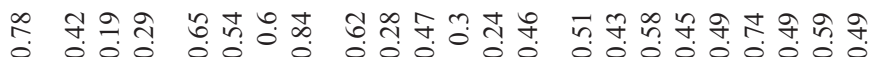
సิ

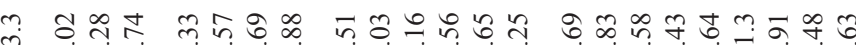

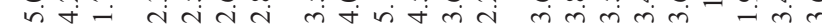

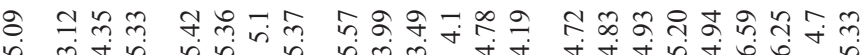
ఫ i - 0 m n i ช.

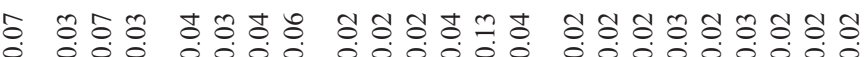

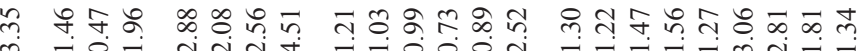
กิ

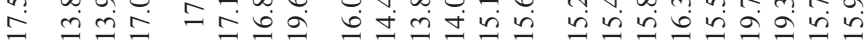

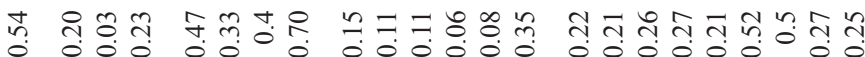
mे

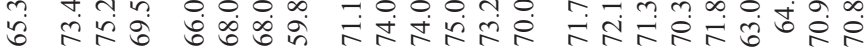
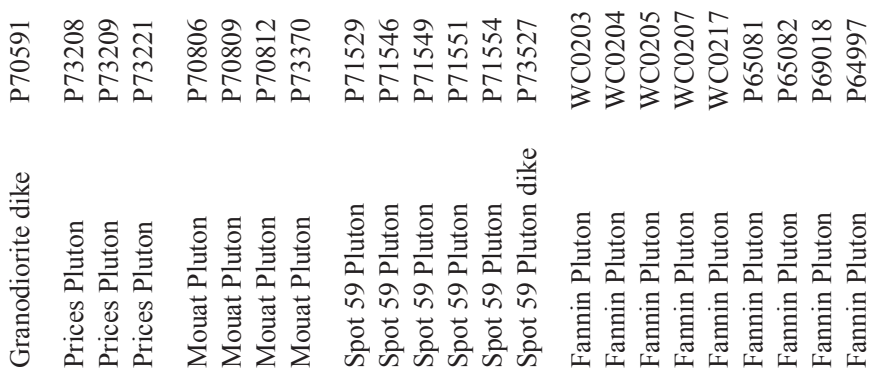
Port Granites, the Brothers, Treble Mountain, Red Head and Indian Island Plutons, and isolated dikes that cut the Lake 773 Pluton, implying that these intrusions are correlatives of either the Rahu and/or the Darran Suites (Fig. 8). Two of the 10 samples from the Indian Island Pluton have HiSY Sr/Y ratios, but the other eight have LoSY Sr/Y ratios, implying that this is the dominant character of the pluton as a whole.

The Rahu and Darran Suites cannot be easily distinguished using XRF geochemical data as compositional fields for the two suites generally overlap (Fig. 8). However, slight enrichment of the Rahu Suite in Sr relative to the Darran Suite at similar $\mathrm{SiO}_{2}$ contents (Waight et al. 1998; Tulloch \& Kimbrough 2003 ) is reflected in the Indian Island, Brothers, North Port, Red Head, and Lake Monk intrusions, although less so in the Revolver Granite (Fig. 8), implying a closer affinity with the Rahu rather than the Darran Suite. The restricted distribution of these LoSY granitoids within the Buller Terrane (except the Lake Monk Granite), the dominance of granitic (sensu stricto) and granodioritic rather than gabbroic through granodioritic compositions also suggest these plutons have a closer affinity with the Rahu rather than the Darran Suite. However, LoSY granitoids in southwest Fiordland were emplaced between c. 140 and $120 \mathrm{Ma}$ and are thus older than the c. $120-105 \mathrm{Ma}$ old granitoids previously included in the Rahu Suite (Waight et al. 1997; Tulloch \& Kimbrough 2003). Sr, Nd, and oxygen isotopic data are required if the correlation of these granitoids is to be clarified further.

\section{INTRUSIONS WITH POORLY CONSTRAINED AGES}

\section{Minor gneissic granitoid bodies in Acheron Passage and outer Breaksea Sound}

Small plugs and narrow layers of gneissic biotite tonalite and granodiorite, ranging in width from decimetres to several hundred metres, intrude metasedimentary rocks along the southeast coast of Resolution Island, the east side of Acheron Passage between 1 and $2.5 \mathrm{~km}$ north of Passage Point, and the northern shore of Breaksea Sound between 2.5 and $3.5 \mathrm{~km}$ west of First Cove. Where intimately interlayered with adjacent metasedimentary rocks, these gneissic granitoid rocks comprise between 20 and $80 \%$ of individual outcrops. Contacts between the gneissic granitoid rocks and adjacent metasedimentary rocks generally parallel the internal foliation in both, and have been folded by open to tight gently NNEplunging folds. A second younger generation of granitoid and pegmatite dikes cut both foliation in the older gneissic granitoids and the gently NNE-plunging folds. Gneissic to massive granodiorite in northeastern Resolution Island is mylonitised adjacent to a major fault near Occasional Cove, but intrudes metasedimentary rock to the west.

Biotite is accompanied by coarse igneous muscovite in c. $80 \%$ of samples, and garnet is present in c. $50 \%$ of samples, implying a peraluminous composition. Accessory minerals include apatite, zircon, allanite, rare opaque oxide, and rare traces of magmatic epidote as rims on allanite. Variable amounts of retrogression to fine white mica and chlorite affect all samples.

These small gneissic granitoid bodies are similar to small bodies of Straight River Granite mapped by Oliver (1980) north of Breaksea Sound. Rb-Sr dating suggests a Paleozoic emplacement age for the Straight River Granite, implying a similar age for these small unnamed bodies of similar granitoid gneiss. Alternatively, they may be minor correlatives of the nearby Cretaceous Indian Island Pluton.

\section{Cascade Pluton}

The 500-1000 m wide, northeast-striking Cascade Pluton (new name, from Cascade Cove) extends from the valley southwest of Cascade Cove across Dusky Sound to Long Island, a distance of c. $9 \mathrm{~km}$ (Fig. 2). At the type locality on the shores of Cascade Cove, the Cascade Pluton comprises incipiently to weakly foliated, locally banded, heterogeneous quartz diorite and quartz monzodiorite with subordinate diorite, gabbro, and minor granodiorite and tonalite (Table 2). Enclaves of finer grained diorite occur in more leucocratic medium-grained granodiorite and tonalite on the southeastern side of Cascade Cove. No overall compositional or mineralogical zonation has been recognised at the scale of the pluton as a whole. Hornblende, biotite, and titanite are ubiquitous, and are commonly accompanied by accessory opaque oxide, epidote, allanite, apatite, and zircon (Table $2)$. Biotite is widely retrogressed to chlorite, and plagioclase to very fine grained sericite (e.g., P65584, P65538). Only rare samples remain largely unaffected by this alteration (e.g., P65542, P70221). A weak foliation defined by aligned mafic minerals and primary centimetre to decimetre-scale compositional banding is present in some outcrops (e.g., P65175). However, remnants of subhedral and interstitial igneous textures are still present throughout the pluton, with some specimens appearing virtually undeformed (e.g., P65584, P70221).

Marginal dikes of the c. $120 \mathrm{Ma}$ Brothers Pluton, and two mica garnet granite derived from either the c. $126 \mathrm{Ma}$ Indian Island Pluton or c. 115 Ma generation \#2 of the Anchor Island Intrusives cut the Cascade Pluton in Cascade Cove, indicating emplacement of the Cascade Pluton before c. $120 \mathrm{Ma}$. The Cascade Pluton cuts adjacent schistose metasedimentary rocks. The continuity and weakly deformed nature of the Cascade Pluton suggest it is younger than generation \#1 of the adjacent Anchor Island Intrusives, implying a Mesozoic rather than Paleozoic age. Pegmatite dikes which cut many units in western Dusky Sound also cut the Cascade Pluton.

\section{Minor diorite plugs and dikes}

Numerous small, unnamed plugs and dikes of massive hornblende diorite, locally accompanied by subordinate hornblende gabbro and quartz diorite, occur throughout the area mapped (Fig. 2). Many are only a few $100 \mathrm{~m}$ across and outcrop over areas smaller than c. $1 \mathrm{~km}^{2}$. Most have been subject only to field examination and basic petrographic analysis since they comprise only c. $1 \%$ of the basement rocks.

Many minor diorite plugs and dikes are superficially similar to all or parts of the larger mafic Only Islands Diorite, Bald, Trevacoon, and Cascade Plutons, and Tower Intrusives, and could be correlatives of one or more of these larger intrusions. Dating indicates that the larger aforementioned mafic intrusions were emplaced at c. 350 , c. 129 , and c. 122-121 Ma. Limited field relationships generally prevent correlation of most of these minor diorite bodies with the three dated phases of mafic plutonism, although some notable exceptions have been mapped.

Dikes and small plugs of diorite and quartz diorite intrude the c. 120.8 Ma old Brothers Pluton in streams that drain 
coastal terraces between Dusky Sound and Chalky Inlet. One of these plugs is 50-200 m thick and several kilometres long (P70339). These bodies are presumably related to the youngest phase of mafic plutonism dated at c. 122-121 Ma. Brittle cataclasis, chlorite-sericite-epidote-quartz-titanite \pm hematite alteration, chlorite-sericite shears, quartz-carbonate veinlets, and traces of sulfide mineralisation (P70347) associated with the Lake Fraser and other faults all locally overprint these dioritic rocks.

Small bodies of massive diorite and quartz diorite on the northern shore of North Port in Chalky Inlet are cut by the c. 128.7 Ma old North Port Granite. Most outcrops are affected by cataclasis and chlorite-sericite alteration associated with the Breaker Point Fault. Protomylonitic fabrics reflecting earlier ductile deformation are locally preserved. Field relationships with the North Port Granite require these dioritic rocks to correlate with either the c. 129 Ma or c. 350 Ma phase of dioritic plutonism.

A range of heterogeneous mafic quartz diorite and tonalitic rocks characterised by marked variations in grain size and composition (e.g., P73791, P73795, P73796, P73798) form part of the basement in the vicinity of Green Islets and the middle reaches of the nearby Grace Burn (Fig. 2). Coarser grained quartz diorite locally contains finer more mafic gabbro enclaves, both of which are cut by veins and dikes of mafic tonalite. Modal biotite exceeds hornblende even in the most mafic rocks. Accessory minerals include opaque oxide, titanite, apatite, allanite, epidote, and zircon. Primary igneous textures are preserved throughout those outcrops not affected by deformation in immediate proximity to faults along the south coast. Chlorite \pm magnetite alteration affects many outcrops around Green Islets, whereas most samples from the Grace Burn are largely unaltered with only trace amounts of sericite and chlorite overprinting plagioclase and biotite, respectively. The biotite-rich nature of these mafic rocks distinguishes them from other mafic plutons in southwest Fiordland. Dikes of Revolver Granite cut these rocks and indicate an age greater than c. $135 \mathrm{Ma}$. Many geochemical characteristics imply a correlation with the c. $350 \mathrm{Ma}$ Tower Intrusives. Chemical analysis of quartz dioritic rocks from near the mouth of the Fred Burn (P70147, 73359) also suggest a correlation with the c. 350 Ma old Tower Intrusives rather than the Cretaceous Bald Pluton or Only Islands Diorite (see above).

Similar small plugs of Cretaceous or Jurassic quartz diorite and diorite intrude the Anchor Island Intrusives and Paleozoic metasediments northeast of The Brothers. A c. 1 $\times 4 \mathrm{~km}$ long body of hornblende biotite diorite (P70290) intrudes the Carboniferous Mt Evans Pluton north of Mt Inaccessible. This body has a distinctive margin of coarser foliated granodiorite with abundant brown biotite and minor muscovite (P70295).

Numerous small diorite bodies intrude Paleozoic metasedimentary rocks and the Big, Houseroof, and Lake 773 Plutons in the eastern Cameron Mountains (Fig. 2). These include a $1.5 \mathrm{~km}^{2}$ ovoid plug $2 \mathrm{~km}$ northwest of Rugged Mount, several 1-5 $\mathrm{km}^{2}$-scale plugs in the catchment of Long Gully (the western tributary of the Waitutu River), minor plugs and dikes on the ridge south of Lake Mouat and on the ridge immediately east of Big River, and a kilometrewide and 3-4 km long, northeast-striking gabbro-diorite plug near the northern end of Lake Poteriteri (P73361, P73363, P73364). The age of these bodies remains largely unconstrained.

\section{BASALT AND ANDESITE DIKES IN WESTERN DUSKY SOUND}

Many subvertical, southeast-striking basalt and andesite dikes from $<1$ to $>10 \mathrm{~m}$ thick cross Five Fingers Peninsula, particularly toward Five Fingers Point. Similar but much less common dikes, with a northeast strike, occur on Parrot Island east of Cascade Cove and on the north coast of Resolution Island. These dikes are variably foliated, ranging from undeformed with well-preserved porphyritic textures, to weakly foliated with transposed contacts against adjacent orthogneiss. However, all clearly postdate foliation development in the host Five Fingers Pluton and Paleozoic metasediments. Hypabyssal dikes are also reported from Fanny Bay (Ward 1984), Gulches Head (Badger 1973), Green Islets (Park 1926; Bishop 1986), and Milford Sound (Post Office Dikes; Wood 1972). None have been dated, although field relationships constrain those at Five Fingers Peninsula to be less than c. 118 Ma old.

The Five Fingers dikes are predominantly dark brown to black, basaltic to andesitic, with some paler possibly dacitic dikes. They were described by Cook in Lindqvist (1984) as equigranular to strongly porphyrytic, with phenocrysts of plagioclase (often zoned), orthopyroxene or hornblende (rimmed with actinolite), and with ophitic or sub-ophitic to intergranular groundmass textures. The groundmass includes ortho- and clinopyroxene, labradorite or bytownite, titanite, and magnetite. Alteration to greenschist facies chlorite, actinolite, and albite assemblages is common, with only rare dikes retaining an unaltered primary mineralogy (e.g., P71334).

\section{DISCUSSION AND CONCLUSIONS}

\section{Extent and history of the Dusky Fault}

Oliver \& Coggon (1979) inferred the presence of a major fault along Dusky Sound, which they referred to as the Dusky Fault, on the basis of marked differences in geological history across the sound. To the north, their "Western Fiordland region" includes granulite facies gneisses structurally overlain by multiply deformed mid to upper amphibolite facies Deep Cove Gneiss (Gibson 1982) metasedimentary rocks that lack primary sedimentary features. To the south they described a "Southwest Fiordland region" of weakly deformed greenschist to amphibolite facies metasedimentary rocks with widely preserved bedding, sedimentary structures and detrital grains, and locally preserved fossils, subsequently included in the Fanny Bay Group (Ward 1984). At the head of Dusky Sound, the Dusky Fault separates Cretaceous Supper Cove Orthogneiss from Devonian Mt Solitary Granodiorite (Davids 1999; Tulloch \& Kimbrough 2003). Juxtaposition of these two units implies significant post-c.130 Ma movement on the Dusky Fault in eastern Dusky Sound.

In western Dusky Sound, the Indian Island and Cascade Plutons and Anchor Island Intrusives cross Dusky Sound, implying no major lateral offset in this area since c. $115 \mathrm{Ma}$. Deep Cove Gneiss metasediments extend as far south as Chalky Inlet (Fig. 2). Only the plunge direction of mesoscopic fold axes varies across the along-strike projection of the Dusky Fault in western Dusky Sound. The Western Fiordland region of Oliver \& Coggon (1979), as defined by the presence of Deep Cove Gneiss, therefore extends south of Dusky Sound 
A

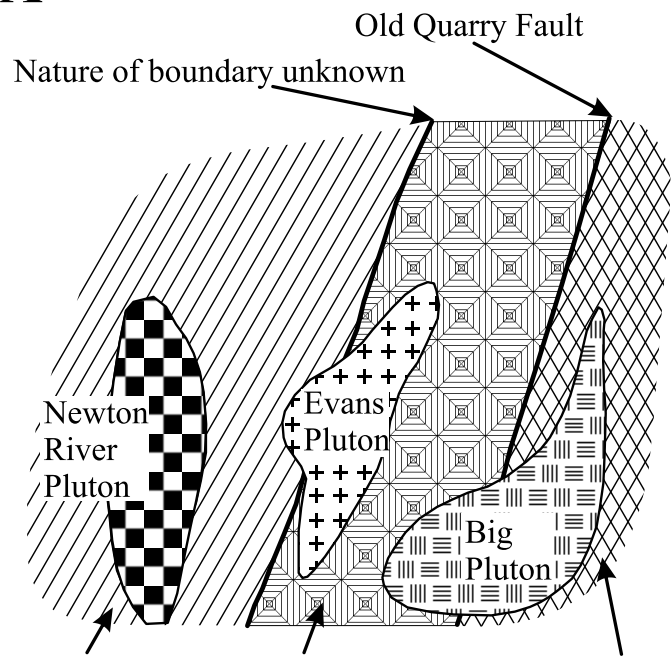

Deep Cove Fanny Bay Group Edgecumbe and Gneiss Buller Terrane Cameron Groups C

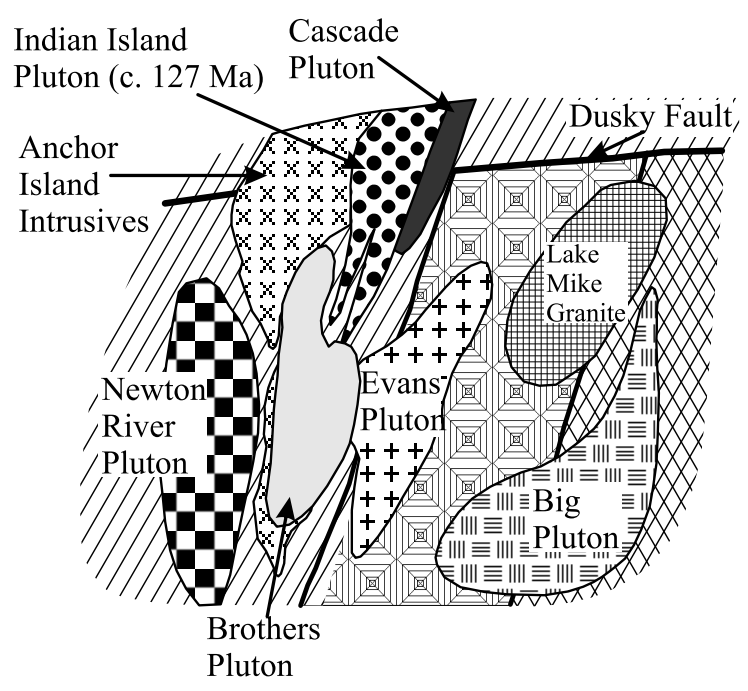

B

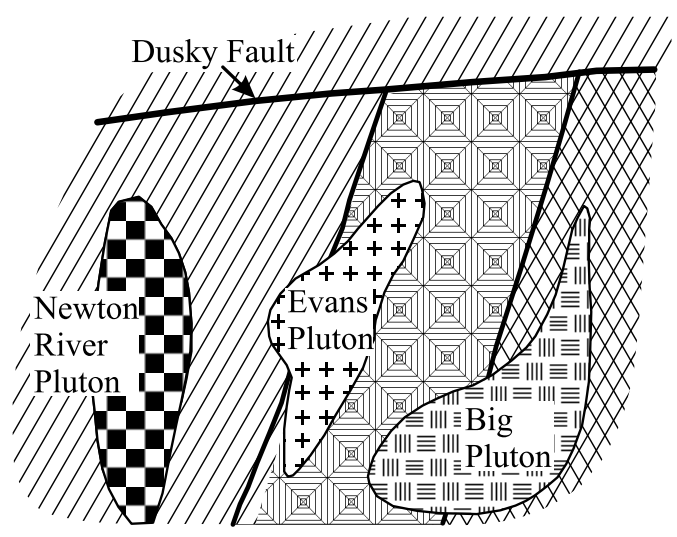

$\mathbf{D}$

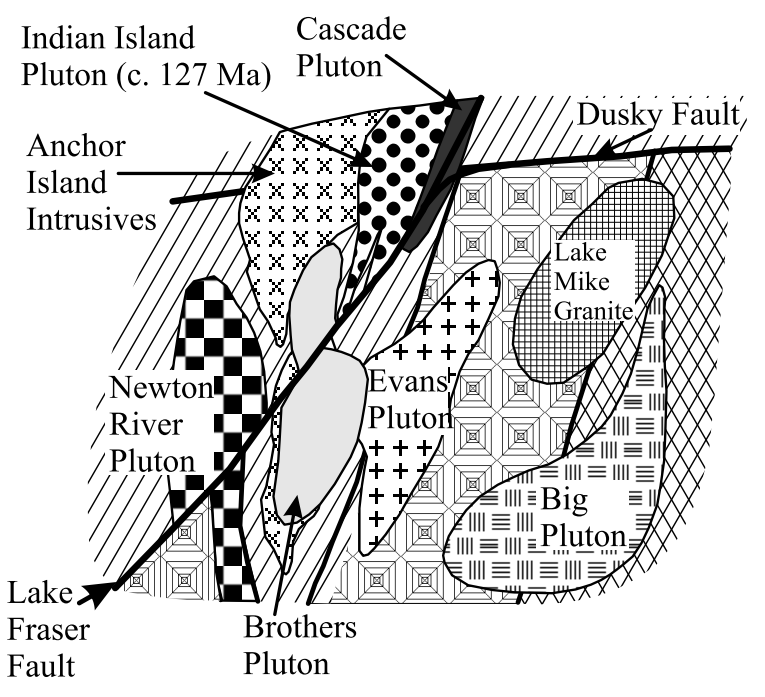

Fig. 9 Schematic sketches illustrating the inferred sequence of events in the vicinity of the Dusky Fault and the boundary between Deep Cove Gneiss (Western Fiordland region) and lower grade sedimentary rocks of the Fanny Bay Group (Southwest Fiordland region) since the mid Paleozoic. A, At c. 350 Ma, the Deep Cove Gneiss, Fanny Bay Group, and Edgecumbe Group were stitched by the Mt Evans and Big Plutons, respectively. B, Subsequent initiation of movement on the Dusky Fault locally juxtaposed the Fanny Bay Group and Deep Cove Gneiss. C, Between c. 127 and 118 Ma, the Dusky Fault was intruded by the Indian Island and Cascade Plutons and Anchor Island Intrusives. D, Initiation of the Lake Fraser Fault and rejuvenation of the eastern part of the Dusky Fault after the mid Cretaceous.

to the Breaker Point Fault on the northern side of Chalky Inlet.

South of Dusky Sound, the boundary between Deep Cove Gneiss metasediments typical of the Western Fiordland region and Fanny Bay Group rocks of the Southwest Fiordland region lies between the Mt Evans and Brothers Plutons (Fig. 2). The original nature of the boundary is unclear. The Mt Evans Pluton may intrude both Deep Cove Gneiss and Fanny Bay Group, implying they were contiguous by c. $350 \mathrm{Ma}$. South of the Breaker Point Fault, North Port Granite intrudes Preservation Formation (Fanny Bay Group) along its eastern margin, but near its western margin it contains xenoliths of garnet-bearing tonalite orthogneiss probably derived from the Western Fiordland region, also implying that the Western and Southwest Fiordland regions were adjacent, in this case at c. $129 \mathrm{Ma}$. The c. $120 \mathrm{Ma}$ Brothers Pluton intrudes Western region Deep Cove Gneiss and the Mt Evans Pluton with its xenoliths of Southwest region Fanny Bay Group, confirming that both regions were "stitched" by the Early Cretaceous.

The Dusky Fault was therefore initiated some time after c. $350 \mathrm{Ma}$ following the joining of the Southwest and Western Fiordland regions by the Mt Evans Pluton (Fig. 9A). The fault offset the contact between the two blocks, as well as the Mt Evans Pluton (Fig. 9B), but only locally juxtaposes 


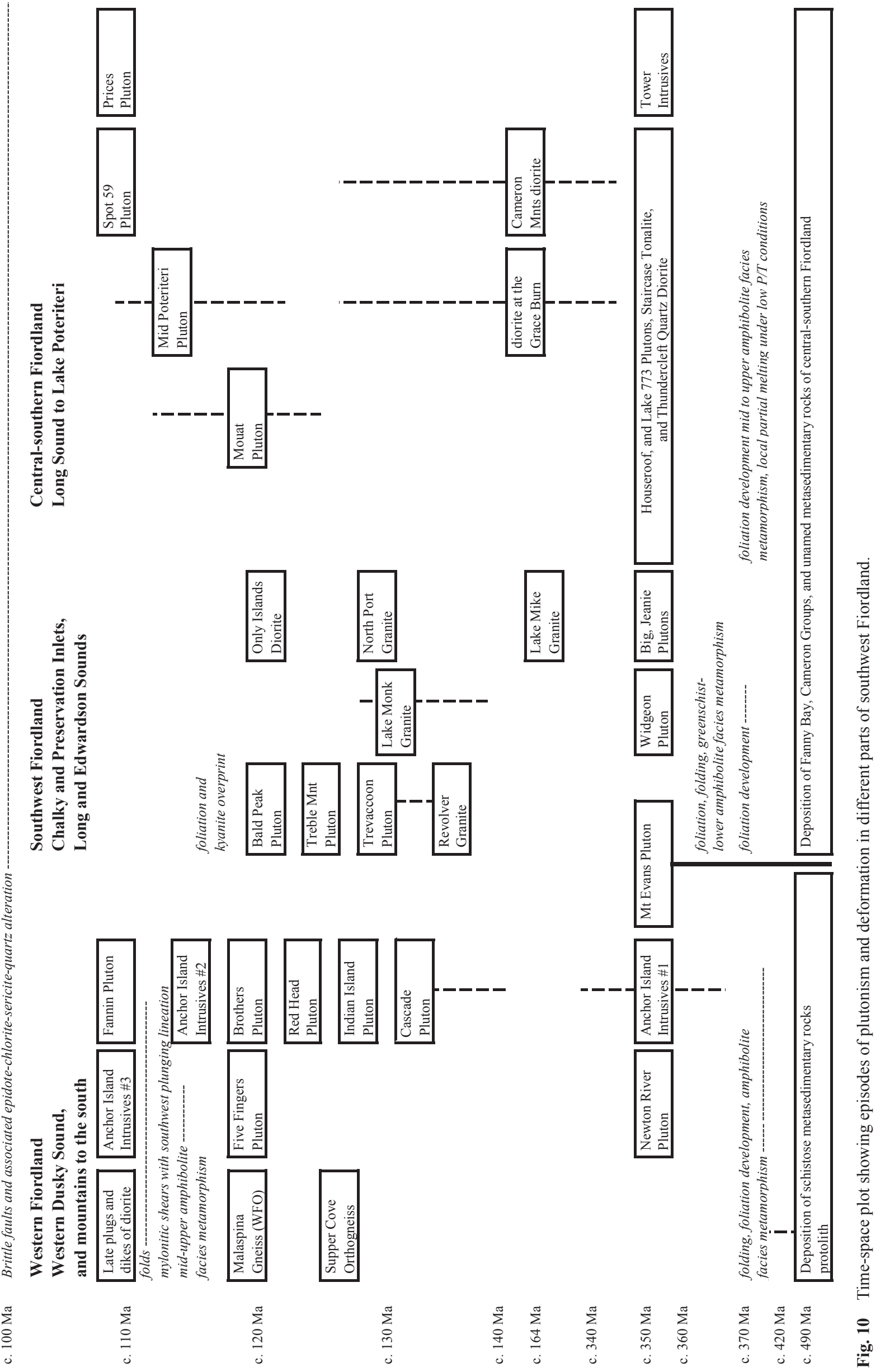




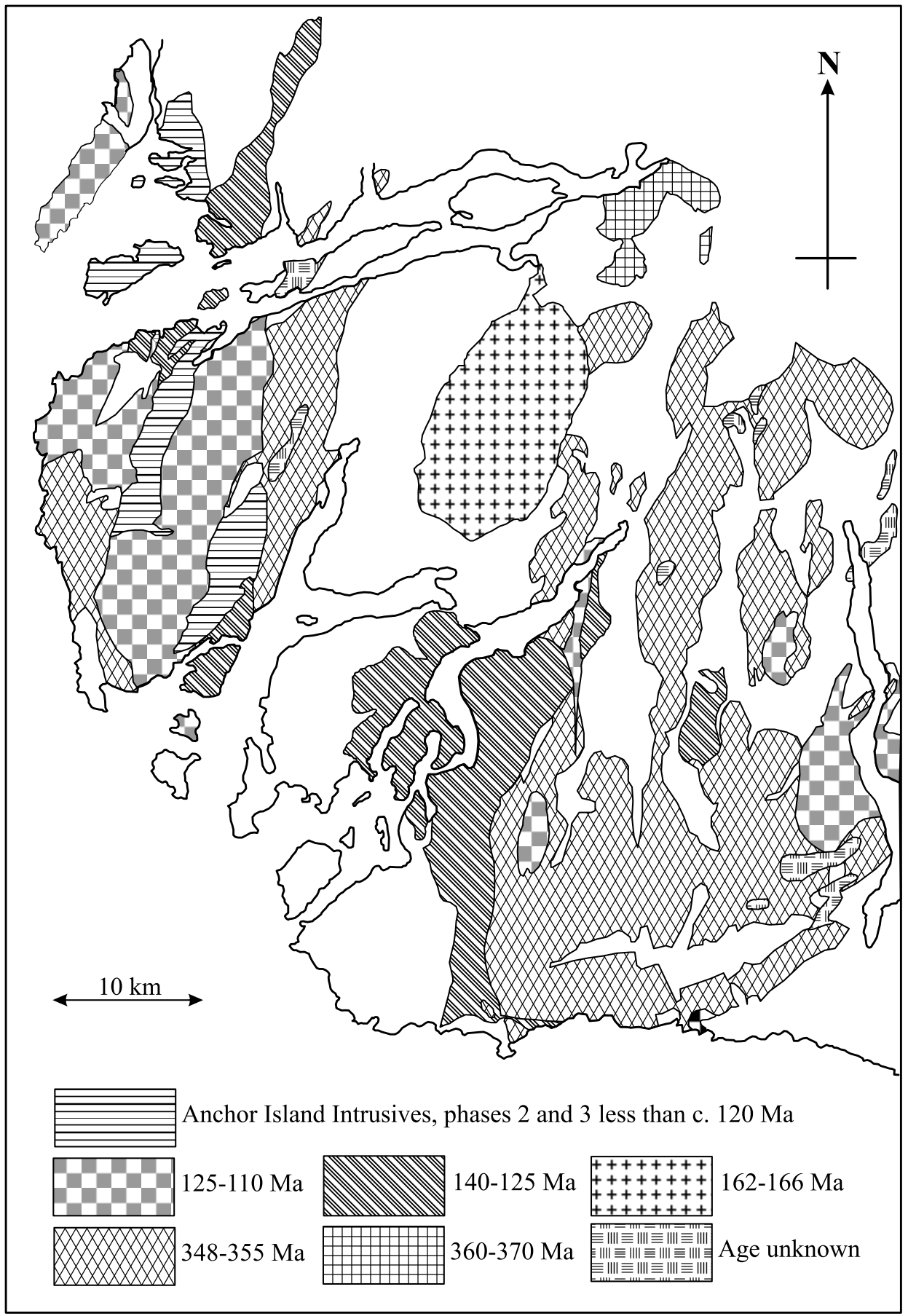

Fig. 11 Summary map illustrating the distribution of plutonic rocks emplaced during Devonian, Carboniferous, Jurassic, and Cretaceous phases of plutonism in southwest Fiordland. (The Devonian rocks immediately south of the head of Dusky Sound are not discussed in this paper; see Davids 1999.)

contrasting metasedimentary rocks from each region. Early Cretaceous plutonism, represented by the Brothers, Indian Island, and Cascade Plutons, and one or more generations of the Anchor Island Intrusives, then cut the proto-Dusky Fault, again stitching rocks with affinities to both regions (Fig. 9C). Subsequent mid to Late Cretaceous movement on the Dusky Fault (Fig. 9D) is probably part of the same episode of faulting as that which led to development of the Lake Fraser and Breaker Point Faults, amongst others. The younger brittle cataclastic deformation on the Dusky Fault in eastern Dusky Sound (Ward 1984) may have been accommodated on the Lake Fraser Fault farther to the west (Fig. 2, 9D), accounting for the continuity of units across western Dusky Sound, west of Cascade Cove.

\section{Regional patterns of c. 350 Ma plutonism in western New Zealand}

About $50 \%$ of the granitoid rocks in southwest Fiordland were emplaced during the Carboniferous with a particularly intense phase of plutonism at c. 355-348 Ma (Fig. 10, 11). Age dating coupled with XRF geochemistry indicates the 354 Ma Big Pluton and other similar intrusions correlate with the S-type Ridge Suite (Tulloch et al. 2003) (Fig. 12). No c. 370-360 Ma plutons of the Karamea and Paringa Suites (Fig. 10, 11), are present in the area mapped, although the c. $350 \mathrm{Ma}$ Mt Evans and Newton River Plutons whose chemistries are similar to Karamea Suite could be regarded as a late rejuvenation of this suite. The small c. 374 Ma Mt Solitary Granodiorite (Ward 
Fig. 12 Summary map illustrating the distribution of different plutonic rock types in southwest Fiordland.

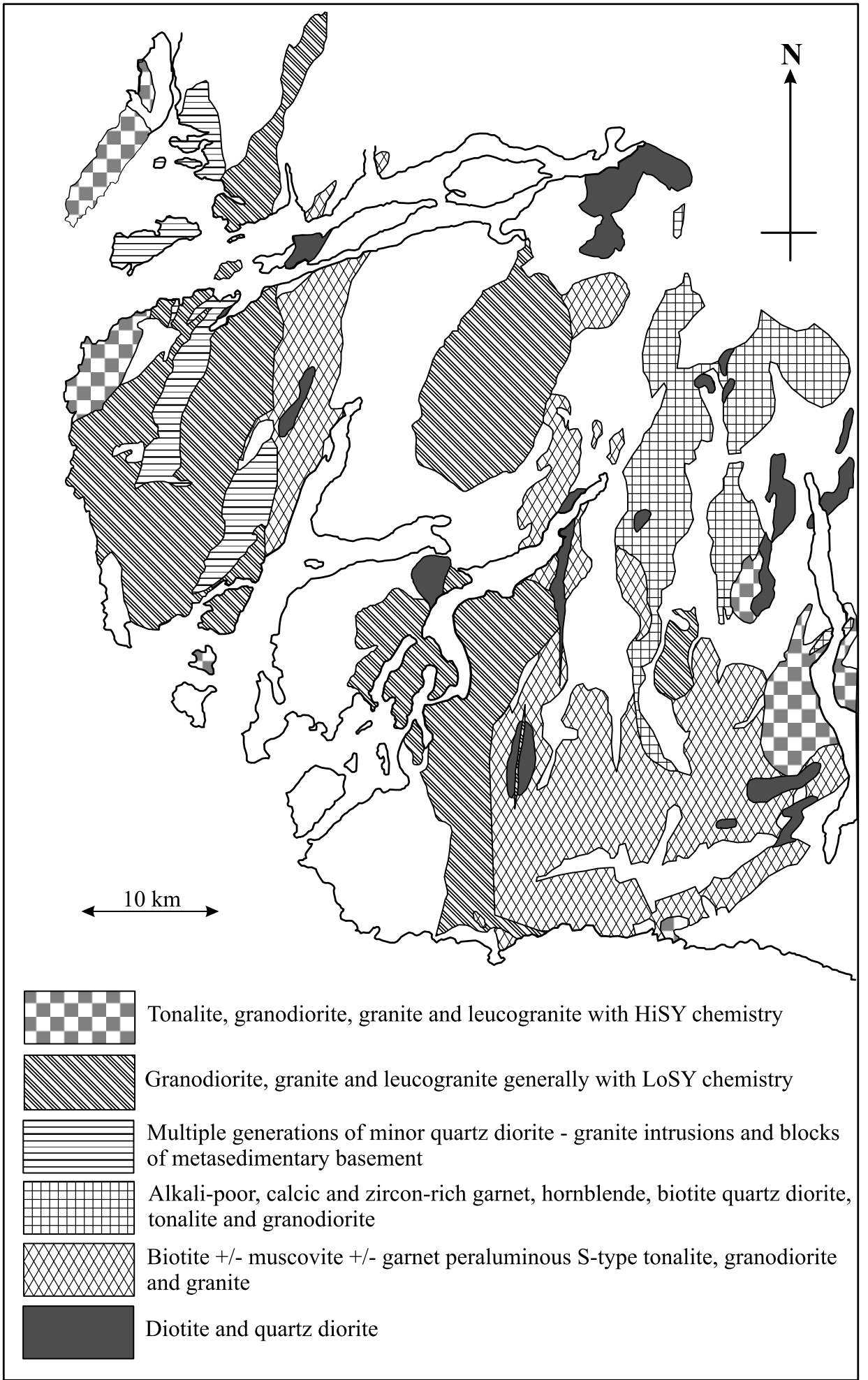

1984; Davids 1999) immediately north of the area mapped (Fig. 2, 11) may be a correlative of one of these suites.

The discovery of large volumes of c. 355-348 Ma plutonic rocks in southwest Fiordland (Fig. 10, 11) coupled with lesser volumes in southern Stewart Island (Allibone \& Tulloch 2004) indicates that this was a major episode of plutonism in the Western Province. Plutonism of this age is widespread in extensions of both the Buller and Takaka Terrane in Fiordland and Stewart Island.

\section{Regional correlation of the Jurassic Lake Mike Granite}

The large Lake Mike Granite is a unique Jurassic pluton in an area otherwise characterised by Paleozoic or Cretaceous plutonism (Fig. 10, 11). It has previously been correlated with the Darran Suite on the basis of its similar age (Kimbrough et al. 1994). However, its homogeneous and relatively leucocratic composition, transitional HiSY/LoSY chemistry (Fig. 8), and inboard location distinguish it from LoSY Darran Suite intrusions of similar age in eastern Fiordland and northern 
Stewart Island, implying it is not a direct correlative. The age of the Lake Mike Granite and its granitoid nature are similar to the South West Arm Pluton in central Stewart Island, although this latter pluton is located farther outboard. Sr/Y ratios for the Lake Mike Granite range between 17 and 50, suggesting it may be an early correlative of the Rahu Suite.

\section{Regional patterns of Jurassic-Cretaceous plutonism in western New Zealand}

Extensive c. 140-120 Ma LoSY granitoid plutonism in southwest Fiordland (Fig. 10, 11, 12) is inferred to correlate with the Rahu Suite of Westland and Nelson. The presence of LoSY granitoid plutons as old as c. $135 \mathrm{Ma}$ in the Buller Terrane of southwest Fiordland implies initiation of Rahu Suite plutonism c. 20 m.y. earlier than previously thought (Tulloch 1983; Muir et al. 1997; Waight et al. 1997, 1998; Tulloch \& Kimbrough 2003). Rahu Suite plutonism in northwest Nelson has generally been interpreted as having formed during the transition from contraction to extension within the New Zealand segment of the Gondwana margin at c. 120-105 Ma (Tulloch 1983; Muir et al. 1997; Waight et al. 1998; Tulloch \& Kimbrough 2003). The presence of similar plutons with a greater age in southwest Fiordland implies that Rahu Suite plutonism may have initiated 15 m.y. earlier while contraction and subduction were still occurring farther to the east along the adjacent margin of Gondwana.

The HiSY Mouat, Spot 59, and Prices Plutons form the southern end of a major north-trending belt of Separation Point Suite HiSY plutons (Fig. 12) that extends at least $100 \mathrm{~km}$ from the south coast past the South Fiord of Lake Te Anau (Fig. 1) (Turnbull et al. 2005; Scott et al. 2005; Allibone, Turnbull, Jongens, Scott unpubl. data). This belt of HiSY intrusions in east-central Fiordland is similar in size and regional structural position to the belt of north-striking HiSY Separation Point Suite plutons in Nelson (Tulloch 1983) and likely represents its along-strike continuation in Fiordland. Further Separation Point Suite HiSY granitoids (Five Fingers, Fannin Plutons) are present in westernmost Fiordland around the mouth of Dusky Sound (Fig. 12). These inboard Separation Point Suite plutons may be correlatives of Separation Point Suite plutons that intrude the Buller Terrane in northwest Nelson (Tulloch 1988; Tulloch \& Rabone 1993; Muir et al. 1997), although in southwest Fiordland they intrude the amphibolite facies Deep Cove Gneiss of unclear terrane affinity.

\section{ACKNOWLEDGMENTS}

Fieldwork in southwest Fiordland was supported by Rodney Russ and crew members on the RV Huia, and we thank Nathan and Aaron Russ especially for their boatmanship. Fieldwork in sometimes trying conditions was assisted by Paul Stenhouse, Duncan Ritchie, Brigid Allan, Tim Hudson, Rob Brown, Yvonne Cook, Jane Forsyth, Hamish Fraser, Trevor Lewis, Luke Milan, Rupert Sutherland, Terry Webb, and Andy West. We would particularly like to thank the Southwest Helicopters team of Wayne Pratt, Doc Sutherland, Sam Gowath, Carol Brown, and Ian and Angela Buick for their logistic support.

We thank colleagues at the Department of Geology, University of Otago, for their contributions to the geology of southwest Fiordland, in particular Chris Ward and Nick Powell for being able to draw extensively on their $\mathrm{PhD}$ theses, and Malcolm Gollan for discussions on the geology of the Long Sound region. We thank Jahandar Ramezani of MIT for his contribution of $\mathrm{U}-\mathrm{Pb}$ geochonological data, which is a major refinement of this study. Various versions of this paper were reviewed by Mark Rattenbury and Richard Jongens of GNS Science. Steve Weaver and George Gibson are also thanked for helpful and constructive reviews of the paper. Rupert Sutherland provided XRF data from granites at West Cape.

Thin sections were made by Neville Orr, and petrological support was provided by John Simes, GNS, Lower Hutt. Mineral separations, data entry, GIS manipulation and drafting services were provided by Belinda Smith Lyttle and Hamish Fraser, GNS Dunedin, and Paul Martin at Geopres Pty Ltd. XRF analyses were provided by Spectrachem Labs Ltd. The mapping on which this paper is based was funded by the Foundation for Research, Science and Technology under contract CO5X0401.

\section{REFERENCES}

Allibone AH 1991. Volcanogenic and granitoid rocks from northwest Stewart Island. New Zealand Journal of Geology and Geophysics 34: 35-50.

Allibone AH, Tulloch AJ 1997. Metasedimentary, granitoid and gabbroic rocks from central Stewart Island, New Zealand. New Zealand Journal of Geology and Geophysics 40: 53-68.

Allibone AH, Tulloch AJ 2004. Geology of the plutonic basement rocks of Stewart Island, New Zealand. New Zealand Journal of Geology and Geophysics 43: 233-256.

Aronson JL 1965. Reconnaissance rubidium-strontium geochronology of New Zealand plutonic and metamorphic rocks. New Zealand Journal of Geology and Geophysics 8: 401-423.

Aronson JL 1968. Regional geochronology of New Zealand. Geochimica et Cosmochimica Acta 32: 669-697.

Badger CP 1973. Geology of Gulches Head Peninsula. Unpublished BSc (Hons) thesis, University of Otago, Dunedin, New Zealand.

Benson WN 1934. The geology of the region about Preservation and Chalky Inlets, southwest Fiordland, New Zealand. Part I. Historical introduction, summary of general geology, and detailed account of the unfossiliferous sediments, igneous rocks, and tectonics. Transactions of the Royal Society of New Zealand 63: 393-432.

Benson WN, Bartrum JA 1935. The geology of the region about Preservation and Chalky Inlets, southwest Fiordland, New Zealand. Part III. Petrology. Transactions of the Royal Society of New Zealand 65: 108-152.

Bishop DG 1986. Sheet B46-Puysegur. Geological map of New Zealand 1:50 000. Map (1 sheet) and notes (36 p.) Wellington, New Zealand, Department of Scientific and Industrial Research.

Bishop DG, Reay A, Koons PO, Turnbull IM 1992. Composition and regional significance of Mid Bay and Mason Bay reefs, Foveaux Strait, New Zealand. New Zealand Journal of Geology and Geophysics 35: 109-112.

Blattner P 1991. The North Fiordland transcurrent convergence. New Zealand Journal of Geology and Geophysics 34: 533-542.

Bradshaw JY 1990. Geology of crystalline rocks of northern Fiordland: details of the granulite facies Western Fiordland Orthogneiss and associated rock units. New Zealand Journal of Geology and Geophysics 33: 465-484.

Collins WJ, Beams SD, White AJR, Chappell BW 1982. Nature and origin of A-type granites with particular reference to southeastern Australia. Contributions to Mineralogy and Petrology 80: 189-200.

Cooper RA 1989. Early Paleozoic terranes of New Zealand. Journal of the Royal Society of New Zealand 19: 73-112.

Cooper RA, Tulloch AJ 1992. Early Paleozoic terranes in New Zealand and their relationships to the Lachlan Fold Belt. Tectonophysics 214: 129-144. 
Cox SC, Allibone AH 1995. Naming of igneous and metamorphic rock units in Antarctica: recommendation by the SCAR Working Group on Geology. Antarctic Science 7: 303306.

Davids C 1999. A thermochronological study of southern Fiordland, New Zealand. Unpublished PhD thesis, Geology Department, Australian National University, Canberra.

Ewing TA 2003. Provenance of the Loch Burn Formation, eastern Fiordland: implications for the Median Tectonic Zone. Unpublished MSc thesis, University of Canterbury, Christchurch, New Zealand.

Frewin MO 1987. Geology of the Anglem Complex, northeastern Stewart Island, New Zealand. Unpublished MSc thesis, University of Otago, Dunedin, New Zealand.

Gibson GM 1982. Stratigraphy and petrography of some metasediments and associated intrusive rocks from central Fiordland, New Zealand. New Zealand Journal of Geology and Geophysics 25: 21-44.

Gibson GM, Ireland TR 1999. Black Giants Anorthosite, New Zealand: a Paleozoic analogue of Archean stratiform anorthosites and implications for the formation of Archean high-grade gneiss terranes. Geology 27: 131-134.

Glazner AF, Bartley JM, Coleman DS, Gray W, Taylor RZ 2004. Are plutons assembled over millions of years by amalgamation from small magma chambers? GSA Today 14: 4-11.

Gollan M 2006. Plutonic petrogenesis and mineralisation in southwest Fiordland. Unpublished MSc thesis, University of Otago, Dunedin, New Zealand.

Gollan M, Palin JM, Faure K, Harris C 2005. Early Cretaceous upper crustal magmatism in southwest Fiordland: geochronological and geochemical highlights. In: Pettinga JR, Wandres AM ed. Geological Society of New Zealand 50th Annual Conference, Kaikoura, 2005, program and abstracts. Geological Society of New Zealand Miscellaneous Publication 119A.

House MA, Gurnis M, Sutherland R, Kamp PJJ 2005. Patterns of late Cenozoic exhumation deduced from apatite and zircon U-He ages from Fiordland, New Zealand. Geochemistry Geophysics Geosystems 6: Q09013.

Hector J 1863. Geological expedition to the west coast of Otago. Otago Provincial Government Gazette 6: 435-538.

Jongens R 2006. Structure of the Buller and Takaka Terrane rocks adjacent to the Anatoki Fault, northwest Nelson, New Zealand. New Zealand Journal of Geology and Geophysics 49: 443-461.

Kimbrough DL, Tulloch AJ, Geary E, Coombs DS, Landis CA 1993. Isotopic ages from the Nelson region of South Island, New Zealand: crustal structure and definition of the Median Tectonic Zone. Techtonophysics 255: 433-438.

Kimbrough DL, Tulloch AJ, Coombs DS, Landis CA, Johnston MR, Mattison JM 1994. Uranium-lead zircon ages from the Median Tectonic Zone, New Zealand. New Zealand Journal of Geology and Geophysics 37: 393-419.

King PL, White AJR, Chappell BW, Allen CM 1997. Characterisation and origin of aluminous A-type granites from the Lachlan Fold Belt, southeastern Australia. Journal of Petrology 38: 371-391.

Landis CA, Coombs DS 1967. Metamorphic belts and orogenesis in southern New Zealand. Tectonophysics 4: 501-518.

Lee WG, Ward CM, Wilson JB 1991. Comparison of altitudinal sequences of vegetation on granite and metasedimentary rocks in the Preservation Ecological District, southern Fiordland, New Zealand. Journal of the Royal Society of New Zealand 21: 261-276.
LeMaitre RW 1989: A classification of igneous rocks and glossary of terms. Subcommission on the Systematics of Igneous Rocks: recommendations of the International Union of Geological Sciences. Oxford, Blackwell. 193 p.

Lindqvist JK 1975. The stratigraphy of the Balleny Group at Puysegur Point, southwest Fiordland, New Zealand. Unpublished MSc thesis, University of Otago, Dunedin, New Zealand.

Lindqvist JK 1984. Basement geology of Five Fingers Peninsula, Dusky Sound, Fiordland. Institute of Geological \& Nuclear Sciences Unpublished Technical File report A44/710.

McCulloch MT, Bradshaw JY, Taylor SR 1987. Sm-Nd and Rb$\mathrm{Sr}$ isotopic and geochemical systematics in Phanerozoic granulites from Fiordland, southwest New Zealand. Contributions to Mineralogy and Petrology 97: 183-195.

Marcotte SB, Klepeis KA, Clarke GL, Gehrels G, Hollis JA 2005. Intra-arc transpression in the lower crust and its relationship to magmatism in a Mesozoic magmatic arc. Tectonophysics 407: $135-163$.

Mortimer N 2004. New Zealand's geological foundations. Gondwana Research 7: 261-272.

Mortimer N, Tulloch AJ 1996. The Mesozoic basement of New Zealand. Geological Society of Australia Extended Abstracts 43: 391-399.

Mortimer N, Tulloch AJ, Ireland TR 1997. Basement geology of Taranaki and Wanganui Basins, New Zealand. New Zealand Journal of Geology and Geophysics 40: 223-236.

Mortimer N, Tulloch AJ, Spark RN, Walker NW, Ladley E, Allibone A, Kimbrough DL 1999: Overview of the Median Batholith, New Zealand: a new interpretation of the geology of the Median Tectonic Zone and adjacent rocks. Journal of African Earth Sciences 29: 257-268.

Muir RJ, Weaver SD, Bradshaw JD, Eby GN, Evans JA 1996a. Geochemistry of the Karamea Batholith, New Zealand and comparisons with the Lachlan Fold Belt granites of SE Australia. Lithos 39: 1-20.

Muir RJ, Ireland TR, Weaver SD, Bradshaw JD 1996b. Ion microprobe dating of Paleozoic granitoids: Devonian magamtism in New Zealand and correlations with Australia and Antarctica. Chemical Geology 127: 191-210.

Muir RJ, Ireland TR, Weaver SD, Bradshaw JD, Waight TE, Jongens R, Eby GN 1997. SHRIMP U-Pb geochronology of Cretaceous magmatism in northwest Nelson-Westland, South Island, New Zealand. New Zealand Journal of Geology and Geophysics 40: 453-463.

Muir RJ, Ireland TR, Weaver SD, Bradshaw JD, Evans JA, Eby GN, Shelley D 1998. Geochronology and geochemistry of a Mesozoic magmatic arc system, Fiordland, New Zealand. Journal of the Geological Society of London 155: 1037-1052.

Oliver GJH 1980. Geology of the granulite and amphibolite facies gneisses of Doubtful Sound, Fiordland, New Zealand. New Zealand Journal of Geology and Geophysics 23: 27-41.

Oliver GJH, Coggon JH 1979. Crustal structure of Fiordland, New Zealand. Tectonophysics 54: 253-292.

Park J 1921. The geology and mineral resources of western Southland, New Zealand. New Zealand Geological Survey Bulletin 23.

Park J 1926. Granite enclosures in quartz-biotite diorite at Green Islets, Southland. Transactions of the Royal Society of New Zealand 56: 384-386. 
Powell NG 2001. Lithostratigraphy and general geology of Cameron Group metasediments, central Southern Fiordland (abstract). In: Lowe DJ, Cooke PJ, Pallentin A ed. Advances in Geosciences. GSNZ annual conference, Hamilton, 2001. Geological Society of New Zealand Miscellaneous Publication 110A. 125.

Powell NG 2006. The geology of central Southern Fiordland. Unpublished $\mathrm{PhD}$ thesis, University of Otago, Dunedin, New Zealand.

Powell NG, Kimbrough DL 1987. On some southern Fiordland acid igneous rocks, with speculations on possible correlatives in West Nelson-Westland. Geological Society of New Zealand Miscellaneous Publication 37A. 94.

Scott J, Cooper A, Palin M, Tulloch A 2005. Petrology and tectonics of eastern Fiordland: some early results. Geological Society of New Zealand Miscellaneous Publication 119A. 78.

Streckeisen A 1976. To each plutonic rock its proper name. Earth Science Reviews 12: 1-33.

Tulloch AJ 1983. Granitoid rocks of New Zealand-a brief review. Geological Society of America Memoir 159: 5-20.

Tulloch AJ 1988. Batholiths, plutons, and suites: nomenclature for granitoid rocks of Westland-Nelson, New Zealand. New Zealand Journal of Geology and Geophysics 31: 505-509.

Tulloch AJ, Braithwaite RL 1986. C7: Granitoid rocks and associated mineralisation of Westland-west Nelson, New Zealand. In: Houghton BF, Weaver SD ed. South Island igneous rocks: tour guides A3, C2 and C7. New Zealand Geological Survey Record 13: 65-92.

Tulloch AJ, Kimbrough DL 2003. Paired plutonic belts in convergent margins and the development of high $\mathrm{Sr} / \mathrm{Y}$ magmatism: Peninsular Ranges batholith of Baja-California and Median batholith of New Zealand. Geological Society of America Special Paper 374: 275-295.

Tulloch AJ, Rabone SDC 1993. Mo-bearing granodiorite porphyry plutons of the Early Cretaceous Separation Point Suite, west Nelson, New Zealand. New Zealand Journal of Geology and Geophysics 36: 401-408.

Tulloch AJ, Ramezani J 2007. U-Pb geochronology of plutons from SW Fiordland. GNS Science unpublished Technical File Report QM417/594.

Tulloch AJ, Kimbrough DL, Faure K, Allibone AH 2003. Paleozoic plutonism in the New Zealand sector of Gondwana. Geoscience Australia Record 2003/14: 123-124.

Turnbull IM, Uruski CI 1995. Geology of the Monowai-Waitutu area, scale 1:50 000. Institute of Geological \& Nuclear Sciences Geological Map 19. 2 sheets +68 p. Lower Hutt, New Zealand, Institute of Geological \& Nuclear Sciences.
Turnbull IM, Allibone AH, Jongens R, Fraser HF, Tulloch AJ 2005. Progress on QMAP Fiordland (abstract). In: Pettinga JR, Wandres AM ed. Geological Society of New Zealand 50th Annual Conference, Kaikoura, New Zealand, programme and abstracts. Geological Society of New Zealand Miscellaneous Publication 119A. 90.

Turner FJ 1935. Metamorphism of the Te Anau series in the region north-west of Lake Wakatipu. Transactions of the Royal Society of New Zealand 65: 329-349.

Waight TE, Weaver SD, Ireland TR, Maas R, Muir RJ, Shelley D 1997. Field characteristics, petrography, and geochronology of the Hohonu Batholith and the adjacent Granite Hill complex, North Westland, New Zealand. New Zealand Journal of Geology and Geophysics 40: 1-17.

Waight TE, Weaver SD, Muir RJ, Maas R, Eby GN 1998. The Hohonu Batholith of North Westland, New Zealand: granitoid compositions controlled by source $\mathrm{H}_{2} \mathrm{O}$ contents and generated during tectonic transition. Contributions to Mineralogy and Petrology 130: 225-239.

Wandres AM, Bradshaw JD 2005: New Zealand tectonostratigraphy and implications from conglomeratic rocks for the configuration of the SW Pacific margin of Gondwana. Geological Society of London Special Publication 246: 179-216.

Wandres AM, Weaver SD, Shelley D, Bradshaw JD 1998. Change from calc-alkaline to adakitic magmatism recorded in the Early Cretaceous Darran Complex, Fiordland, New Zealand. New Zealand Journal of Geology and Geophysics 41: 1-14.

Ward CM 1984. Geology of the Dusky Sound area, Fiordland with emphasis on the structural-metamorphic development of some porphyroblastic staurolite pelites. Unpublished $\mathrm{PhD}$ thesis, University of Otago, Dunedin, New Zealand.

Ward CM 1986. The Fanny and Goodyear terranes of southern Fiordland and their relations with west Nelson. In: Lower Paleozoic Workshop. University of Canterbury. Pp. 46-47.

Wood BL 1960. Sheet 27-Fiord. Geological map of New Zealand 1:250 000. Wellington, New Zealand, Department of Scientific \& Industrial Research.

Wood BL 1972. Metamorphosed ultramafites and associated formations near Milford Sound, New Zealand. New Zealand Journal of Geology and Geophysics 15: 88-128.

Wood BL, Grindley GW 1954. Southern Fiordland between Lake Hauroko and Dusky Sound. Unpublished NZGS Techfile report B44/710. GNS Science, Dunedin. 